\title{
Network Dynamics, Synchronization, and Self-Propelled Particles in Chemical Systems
}

Hua Ke

West Virginia University

Follow this and additional works at: https://researchrepository.wvu.edu/etd

\section{Recommended Citation}

Ke, Hua, "Network Dynamics, Synchronization, and Self-Propelled Particles in Chemical Systems" (2013). Graduate Theses, Dissertations, and Problem Reports. 4975.

https://researchrepository.wvu.edu/etd/4975

This Dissertation is protected by copyright and/or related rights. It has been brought to you by the The Research Repository @ WVU with permission from the rights-holder(s). You are free to use this Dissertation in any way that is permitted by the copyright and related rights legislation that applies to your use. For other uses you must obtain permission from the rights-holder(s) directly, unless additional rights are indicated by a Creative Commons license in the record and/ or on the work itself. This Dissertation has been accepted for inclusion in WVU Graduate Theses, Dissertations, and Problem Reports collection by an authorized administrator of The Research Repository @ WVU.

For more information, please contact researchrepository@mail.wvu.edu. 


\title{
Network Dynamics, Synchronization, and Self-Propelled
}

\section{Particles in Chemical Systems}

\author{
Hua Ke
}

\begin{abstract}
DISSERTATION
Submitted to the Eberly College of Arts and Sciences at West Virginia University in Partial Fulfillment of the Requirements for the Degree of

Doctor of Philosophy

in

Chemistry
\end{abstract}

Kenneth Showalter, Ph.D., Chairman

Charles Jaffé, Ph.D.

Terry Gullion, Ph.D.

Justin Legleiter, Ph.D.

Martin Ferer, Ph.D.

Morgantown, West Virginia

2013

Keyword: BZ Reaction, Neural Network, Chemical Neural Network, Synchronization, Phase Kink, Brownian Motion, Self-Propelled Particles 


\title{
Abstract
}

\section{Network Dynamics, Synchronization, and Self-Propelled Particles in Chemical Systems}

\author{
Hua Ke
}

Neural networks are a class of biological networks of great importance. They are a key component of the central nervous system that coordinates body functions. The exploration of the detailed mechanism of biological neural networks remains extremely active. Inspired by the structure of biological neural networks, artificial neural networks have been designed to solve a variety of problems in pattern recognition, prediction, optimization and control. However, few studies have been reported that explore the dynamics of biological neural networks using chemical systems. As part of this thesis, an experimentally trainable network based on the photosensitive Belousov-Zhabotinsky reaction is developed, where the individual node is a catalyst loaded micro-particle. The interactions between nodes in the network are created by arranging links with different weights, similar to the excitable and inhibitory synapses in biological neural networks. The distribution of the weights of the excitable links has been studied. The results indicate that a stable distribution of the weights is exhibited.

Synchronization in coupled nonlinear oscillators is a remarkable and ubiquitous phenomenon in nature. Application of periodic global feedback to oscillators allows the creation of new kinds of 
wave patterns with the coexistence of stable phase states. In experiments with the photosensitive BZ reaction, periodic global feedback is implemented by varying the illumination intensity. In a 1:1 frequency-locked entrainment, $2 \pi$ phase fronts called phase kinks have been observed in the photosensitive BZ reaction. Generally, a phase kink represents the existence of stable phase differences, propagating as an analog of traveling waves in 2D excitable media. By modifying the conditions of local forcing, the experiments show that a phase kink can be trapped to form a closed pattern.

Self-propulsion is an essential feature of many living systems. There are numerous realizations of self-propelled particles in biological systems, such as the bacteria Listeria monocytogenes in cells. Such biological phenomena inspire the creation of artificial self-propelled particles. Recently, nonbiological micro- to nanoscale particles, that convert chemical energy into translational motion, have been investigated. Studies show that Pt-coated polystyrene particles, coated on one hemisphere with Pt, exhibit self-propulsion in dilute $\mathrm{H}_{2} \mathrm{O}_{2}$ solutions. Here, we experimentally study the dynamical behavior of silica particles that are asymmetrically coated with $\mathrm{Pt}$ in $\mathrm{H}_{2} \mathrm{O}_{2}$ solutions, similar to Pt-coated polystyrene particles. The focus of our study is on the particle orientation with respect to the direction of motion. This is investigated using velocity autocorrelation and propulsion direction analyses. 


\section{Acknowledgements}

I sincerely appreciate my advisor, Dr. Kenneth Showalter. He provided me a great research atmosphere to pursue my research; his great patience, extraordinary enthusiasm and broad knowledge allowed me to solve many problems during my research. My special thanks to Dr. Mark Tinsley. His research capability, computer skill, and brilliant insights and perspectives into experimental phenomena always inspired me. My deep appreciation goes to Dr. R. Lloyd Carroll and Dr. Shengrong Ye, whose excellent ideas helped me achieve important results in my research. I also want to express my gratitude to Dr. Zhaoyang Huang, Dr. Fang Wang, Dr. Calistus Ngonhala, Simbarashe Nkomo, Desmond Yengi, Darrell Collison, and Razan Snari for their kind help through my graduate student life.

My greatest thanks go to my family: my grandmother, my parents, my sister, and my wife, Yingjiao Liang. Their support gives me the biggest motivation, and always encourages me to conquer the problems in my study and move forward to the destination. 


\section{Contents}

$\begin{array}{lll}\text { Abstract } & \text { i }\end{array}$

$\begin{array}{ll}\text { Acknowledgements } & \text { iii }\end{array}$

Contents

$\begin{array}{lc}\text { List of Figures } & \text { vii }\end{array}$

$\begin{array}{lc}\text { List of Tables } & \text { X }\end{array}$

1 The Belousov-Zhabotinsky (BZ) Reaction $r$

1.1 The Oregonator Model . . . . . . . . . . . . . . . . . . . . . 2

1.2 Effect of Light on the BZ Reaction . . . . . . . . . . . . . . . . . 6

1.3 Modified Oregonator Model for the Photosensitive BZ Reaction . . . . . . . . . 7

1.4 Pattern Formation in the BZ Reaction . . . . . . . . . . . . . . . . . . 9

1.4.1 Patterns without External Forcing . . . . . . . . . . . . . . 9

1.4.2 Patterns with External Forcing . . . . . . . . . . . . . . . 11

1.4.3 Effect on Dynamics of Trigger Waves . . . . . . . . . . . . . . . . 12

2 Neural Network Model System 23

2.1 Introduction . . . . . . . . . . . . . . . . . . . . . . 24 
2.1 .1 Concept of Network . . . . . . . . . . . . . . . . . . . . . 24

2.1.2 Natural Networks . . . . . . . . . . . . . . . . . . . . . . . . 25

2.2 Biological Neural Networks . . . . . . . . . . . . . . . . . . . . 26

2.2 .1 Neurons and Spikes . . . . . . . . . . . . . . . . . . . . . 26

2.2 .2 Hebbian Learning . . . . . . . . . . . . . . . . . . . . . . . 30

2.2.3 Effects of Synaptic Connections on Memory Storage . . . . . . . . . . . . . 31

2.3 Artificial Neural Networks . . . . . . . . . . . . . . . . . . . . . . . . . 32

2.3.1 Theoretical Models of Neurons . . . . . . . . . . . . . . . . . . . . . . 32

2.3.2 Network Models Inspired by Biological Neural Networks . . . . . . . . . . 33

2.3.3 Spike-Timing-Dependent Plasticity (STDP) . . . . . . . . . . . 35

3 A Trainable Chemical Neural Network: Experiments and Simulations 43

3.1 Experimental Setup . . . . . . . . . . . . . . . . . . . . . . . . . 44

3.1.1 Preparation of the Chemical System . . . . . . . . . . . . . . . 44

3.1 .2 The Instrumentation Setup $\ldots \ldots$. . . . . . . . . . . . . 46

3.1 .3 The Network Setup . . . . . . . . . . . . . . . . . . . . . . . . 46

3.2 Experimental Results . . . . . . . . . . . . . . . . . . 50

3.3 Simulations . . . . . . . . . . . . . . . . . . . . . . . . 58

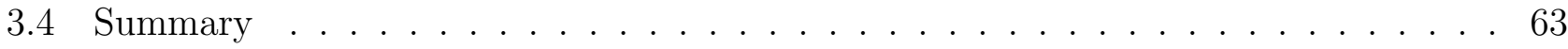

4 Phase Kinks in the Belousov-Zhabotinsky Reaction 68

4.1 Introduction . . . . . . . . . . . . . . . . . . . . . . . . . 69

4.2 Experimental Design . . . . . . . . . . . . . . . . . . 73

$4.2 .1 \quad$ Experimental Setup . . . . . . . . . . . . . . . . . . . 73

4.2.2 Preparation of the Chemical System _ . . . . . . . . . . . 76 
4.3 Experimental Results . . . . . . . . . . . . . . . . . . . . . . . 78

4.3.1 The Background Oscillation . . . . . . . . . . . . . . . 78

4.3 .2 Creation of Phase Kinks . . . . . . . . . . . . . . . . . . . . . . 79

4.3 .3 Trap of Phase Kinks . . . . . . . . . . . . . . . . . . . . . 83

4.4 Simulations . . . . . . . . . . . . . . . . . . . . . . . . . 89

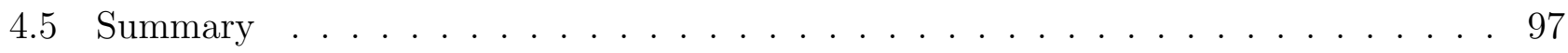

5 Motion Analysis of Self-Propelled Pt-Silica Particles in Hydrogen Peroxide

Solutions

5.1 Introduction . . . . . . . . . . . . . . . . . . . . 105

$5.2 \quad$ Experimental Setup . . . . . . . . . . . . . . . . . . . . . . . 106

5.2.1 Preparation of Silica Particles Half-Coated with Platinum _ . . . . . . . 107

5.2.2 Data Collection and Particle Tracking Analysis . . . . . . . . . . . . 108

5.3 Experimental Results . . . . . . . . . . . . . . . . . . . . . . . . 108

5.4 Summary . . . . . . . . . . . . . . . . . . . . . . 123 


\section{List of Figures}

1.1 Three states described by the $u-v$ phase plane from the two-variable Oregonator model with $q=0.02$ and different $f$ values . . . . . . . . . . . . . . . 5

1.2 The effect of light intensity on the excitability of the photosensitive BZ reaction . 8

2.1 Three typical network structures with the number of nodes $n=20 \ldots \ldots$

2.2 The food web in Little Rock Lake, Wisconsin . . . . . . . . . . . . . . . . . . 25

2.3 Diagram of a typical motor neuron . . . . . . . . . . . . . . . . . . . . 27

2.4 A schematic diagram of typical neuron spikes . . . . . . . . . . . . . . . . . . 28

2.5 C. elegans and its neural network . . . . . . . . . . . . . . . . . . 29

2.6 The concept of a firing threshold . . . . . . . . . . . . . . . . . . . . 33

2.7 The back-propagation network for a linear associative memory . . . . . . . . . . . . 34

3.1 Experimental setup for the BZ network . . . . . . . . . . . . . . . . . . 45

3.2 Particles reacting in BZ solutions as the nodes in the chemical network . . . . . . . 47

3.3 Arrangement of links in the chemical system . . . . . . . . . . . . . . . . . 49

3.4 Diagram of training process . . . . . . . . . . . . . . . . . . . . . 50

3.5 Effect of links on the oscillator illumination . . . . . . . . . . . . . . . . . 51

3.6 Firing events in the photosensitive Oregonator model . . . . . . . . . . . . . . . 52

3.7 The behaviors of nodes with different illumination patterns . . . . . . . . . . . 53 
3.8 The period of oscillation in a network illuminated with $\phi_{l o w}=0.08 \mathrm{~mW} \mathrm{~cm}^{-2}$ and in an STDP network . . . . . . . . . . . . . . . . . . . 55

3.9 Experimental system firing pattern . . . . . . . . . . . . . . . 55

3.10 The activity of the STDP system as a function of time . . . . . . . . . . 56

3.11 The distribution of weights of the excitatory links at different experimental times . 57

3.12 A patchy wave of activity propagating in the BZ network with STDP . . . . . . . 59

3.13 Probability of sustained behavior as a function of excitatory link weight . . . . . . 60

3.14 Numerical system firing pattern . . . . . . . . . . . . . . . . . 61

3.15 The STDP rule applied to the numerical system . . . . . . . . . . . . . 63

4.1 Profiles of the oscillation amplitude modulus (a) and of the phase (b) in traveling phase kinks with global feedback . . . . . . . . . . . . . . . 69

4.2 Schematic diagram of a phase kink in a $2 \mathrm{D}$ medium . . . . . . . . . . . . 70

4.3 The complex dynamics of phase kinks in different regions of the Arnold tongue . . . 72

4.4 The experimental setup for phase kinks in the BZ reaction . . . . . . . . . . . . . 74

4.5 A sketch of periodic forcing illumination as a function of time . . . . . . . . 75

4.6 The frequency of the background oscillator in the oscillatory BZ reaction as a func-

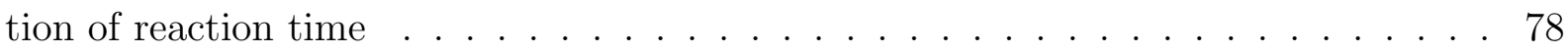

4.7 The entrainment of the background oscillations . . . . . . . . . . . . . . . 80

4.8 A phase kink propagating in the oscillatory BZ reaction . . . . . . . . . . . . 82

4.9 Plots (a) and (b) show the gray level at points A and B in Fig. 4.8, respectively, as

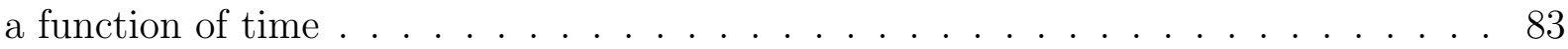

4.10 Schematic diagram for trapping a phase kink in the oscillatory BZ reaction . . . . . 84

4.11 Snapshots of trapping a phase kink in the oscillatory BZ reaction . . . . . . . . 85

4.12 An unsuccessful trapping of a phase kink in the oscillatory BZ reaction . . . . . . 87 
4.13 Disturbance of pacemakers in the process of trapping a phase kink in the oscillatory

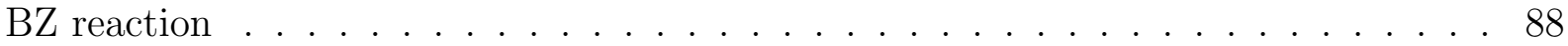

4.14 Snapshots of a phase kink calculated using the photosensitive Oregonator model . . 90

4.15 Collapse of the local heterogeneity in the photosensitive Oregonator model with external forcing . . . . . . . . . . . . . . . . . . . . . 92

4.16 Amplitude change of phase kink during a period . . . . . . . . . . . . . . . 93

4.17 Arnold tongue with 1:1 frequency-locked entrainment in the photosensitive Oregonator model . . . . . . . . . . . . . . . . . . . . . . 94

4.18 Effect of amplitude and frequency of external forcing on the velocities of phase kinks in the photosensitive Oregonator model . . . . . . . . . . . . . . . . . 95

4.19 Trap of a phase kink in the photosensitive Oregonator model . . . . . . . . . . . 96

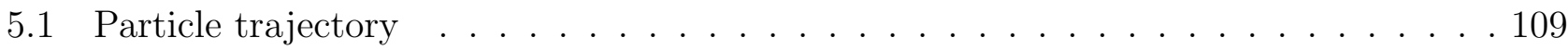

5.2 Plot of normalized occurrence distribution . . . . . . . . . . . . . . . . . 110

5.3 Distribution of directional angle . . . . . . . . . . . . . . . . . . . . 112

5.4 Normalized velocity autocorrelation function for uncoated silica and half-coated Ptsilica particles in water and $\mathrm{H}_{2} \mathrm{O}_{2}$ solutions . . . . . . . . . . . . . . . 113

5.5 Average speed $(\mathrm{O})$ of three half-coated Pt-silica particles . . . . . . . . . . 115

5.6 Maximum projection of 120 frames of a half-coated Pt-silica particle traveling in a $10.0 \% \mathrm{w} / \mathrm{w}_{2} \mathrm{O}_{2}$ solution. . . . . . . . . . . . . . . . 116

5.7 Distribution of the step length $\Delta$ for (a) silica and (b) Pt-silica particles . . . . . 119

5.8 Translational diffusion coefficient $D_{\text {tran }}$ as a function of the observation time interval

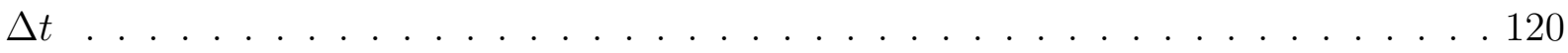




\section{List of Tables}

5.1 Values of $D_{\text {tran }}, \tau_{R}$, and $V$ from Step Length Probability Distribution . . . . . 121

5.2 Values of $D_{\text {tran }}, \tau_{R}$, and $V$ from Mean-Squared Displacement . . . . . . . . 123 


\section{Chapter 1}

\section{The Belousov-Zhabotinsky (BZ) Reaction}

The Belousov-Zhabotinsky (BZ) reaction was discovered by B. P. Belousov in 1951 and was extensively studied by A. M. Zhabotinsky beginning in 1961 [1]. In its classical form, the BZ reaction involves the oxidation of malonic acid by bromate catalyzed by a metal ion such as $\mathrm{Ce}^{3+}$ or a metal complex ion such as $\mathrm{Fe}(\mathrm{phen}){ }_{3}^{2+}$ in acidic aqueous solution [2]. The overall reaction is given by

$$
2 \mathrm{BrO}_{3}^{-}+3 \mathrm{CH}_{2}(\mathrm{COOH})_{2}+2 \mathrm{H}^{+} \rightarrow 2 \mathrm{BrCH}(\mathrm{COOH})_{2}+3 \mathrm{CO}_{2}+4 \mathrm{H}_{2} \mathrm{O}
$$

The reaction involves many intermediates, and, as it progresses, the concentrations of the intermediates undergo oscillations.

The BZ reaction has become one of the most widely studied chemical reactions and is a model system for more complex biological and biochemical oscillatory systems. Here, one of the most important models for the BZ reaction, the Oregonator model, is described in detail, and the reaction-diffusion behavior in the $\mathrm{BZ}$ reaction is also introduced. In Chapter 3, we will study the dynamics of a neural network based on the BZ reaction. In Chapter 4, a type of synchronization pattern in the BZ reaction, called phase kinks, will be illustrated. 


\subsection{The Oregonator Model}

A detailed chemical mechanism of the BZ reaction, involving eleven elementary steps, was proposed by Field, Körös and Noyes in 1972, which became known as the FKN mechanism [3]. The step that is key to the behavior of the system is the autocatalytic production of bromous acid, $\mathrm{HBrO}_{2}$. For each molecule of $\mathrm{HBrO}_{2}$ consumed in the step, two molecules are produced. The rapid production of $\mathrm{HBrO}_{2}$ continues until another process involving the metal ion catalyst regenerates bromide, which consumes bromous acid and inhibits the autocatalysis. As long as there is sufficient bromide, the bromous acid is not available for the autocatalytic step. Since the major reactant bromate consumes bromide, the bromide concentration eventually decreases to a critical level. The autocatalytic step again occurs, oxidizing the metal ion catalyst, which is followed by the process that reduces the metal ion catalyst and produces bromide $[4,5]$. An oscillation cycle is summarized by the following steps: (1) bromide concentration is high, and bromide is consumed; (2) bromide concentration is low, and autocatalytic bromous acid production takes place, during which the catalyst is oxidized; (3) the oxidized catalyst is reduced, producing bromide.

In 1974, Field and Noyes proposed a distillation of the FKN mechanism, a three-variable scheme known as the Oregonator [4]. The Oregonator is typically written using the following definitions:

$$
\begin{array}{ll}
A=\left[\mathrm{BrO}_{3}^{-}\right], & P=[\mathrm{HOBr}], \\
Y=\left[\mathrm{Br}^{-}\right], & Z=\left[\mathrm{Ce}^{4+}\right] .
\end{array}
$$


The steps of the Oregonator are assumed to be irreversible and can be written as [6, 7]:

$$
\begin{gathered}
A+Y \stackrel{k_{7}}{\rightarrow} X+P, \\
X+Y \stackrel{k_{2}}{\rightarrow} 2 P, \\
A+X \stackrel{k_{3}}{\rightarrow} 2 X+2 Z, \\
2 X \stackrel{k_{5}}{\rightarrow} A+P, \\
Z \stackrel{k_{5}}{\rightarrow} h Y .
\end{gathered}
$$

Tyson [8] included bromomalonic acid as a reactant in step R5; however, here its concentration is assumed to be constant and is included in $k_{5}$. From reactions (R1)-(R5), the rate equations are given by

$$
\begin{aligned}
& \frac{d X}{d \tau}=k_{1} A Y-k_{2} X Y+k_{3} A X-2 k_{4} X^{2}, \\
& \frac{d Y}{d \tau}=-k_{1} A Y-k_{2} X Y+h k_{5} Z \\
& \frac{d Z}{d \tau}=2 k_{3} A X-k_{5} Z .
\end{aligned}
$$

The following definitions suggested by Tyson and Fife [8, 9] are used to non-dimensionalize the rate equations:

$$
\begin{aligned}
u & =\left(\frac{2 k_{4}}{k_{3} A}\right) X, & v & =\left(\frac{k_{4} k_{5}}{\left(k_{3} A\right)^{2}}\right) Z, \\
w & =\left(\frac{k_{2}}{k_{3} A}\right) Y, & \epsilon & =\frac{k_{5}}{k_{3} A}, \\
\epsilon^{\prime} & =\frac{2 k_{4} k_{5}}{k_{2} k_{3} A}, & q & =\frac{2 k_{1} k_{4}}{k_{2} k_{3}}, \\
t & =k_{5} \tau, & f & =2 h .
\end{aligned}
$$


Using these definitions, the rate equations of the Oregonator can be written as

$$
\begin{aligned}
\epsilon \frac{d u}{d t} & =q w-u w+u-u^{2}, \\
\frac{d v}{d t} & =u-v, \\
\epsilon^{\prime} \frac{d w}{d t} & =-q w-u w+f v .
\end{aligned}
$$

The three-variable model can be reduced to a two-variable model by solving for the steady state variable $w$ in Eq. 1.3c, since $\epsilon^{\prime}<<\epsilon[9,10]$

$$
w=\frac{f v}{u+q}
$$

which can be used to replace $w$ in Eq. 1.3a:

$$
\begin{aligned}
& \frac{\partial u}{\partial t}=\frac{1}{\epsilon}\left[u-u^{2}-(f v) \frac{u-q}{u+q}\right], \\
& \frac{\partial v}{\partial t}=u-v .
\end{aligned}
$$

The two-variable model preserves the qualitative behavior of the BZ reaction and makes a simple phase-plane description possible $[8,10,11]$. The different states in the BZ reaction described by the two-variable Oregonator model based on three different values of $f$ are shown in Fig. 1.1. The curved and straight dashed lines in the figure represent the $\frac{\partial u}{\partial t}=0$ and $\frac{\partial v}{\partial t}=0$ nullclines, respectively. The crossing point of the two nullclines represents the steady state of the system. In Fig. 1.1a and 1.1c, the system will return to the steady state after a perturbation since the intersection point lies on the left or the right branch of the $\frac{\partial u}{\partial t}=0$ nullcline. However, the system is unstable in Fig. 1.1b, since the intersection is on the middle branch of the $u$-nullcline, and a limit cycle is formed after a small perturbation [4]. 


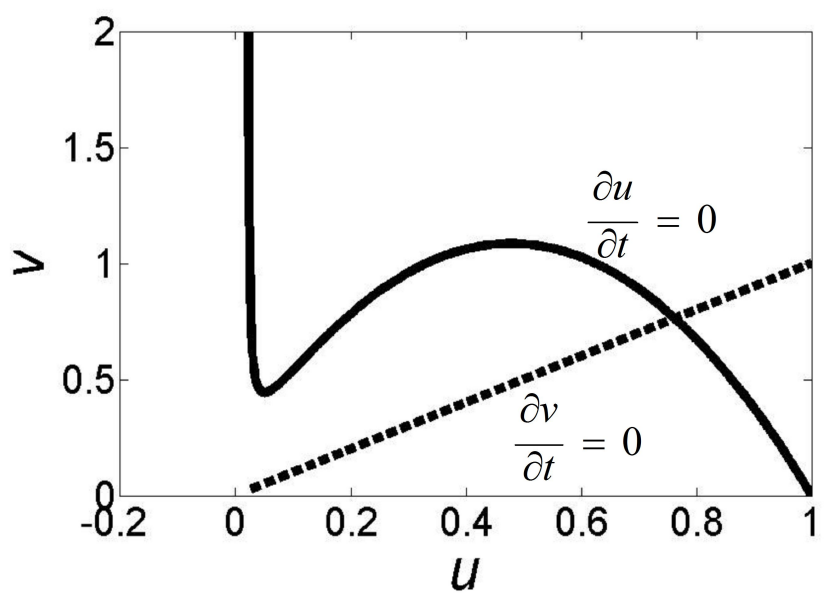

(a)

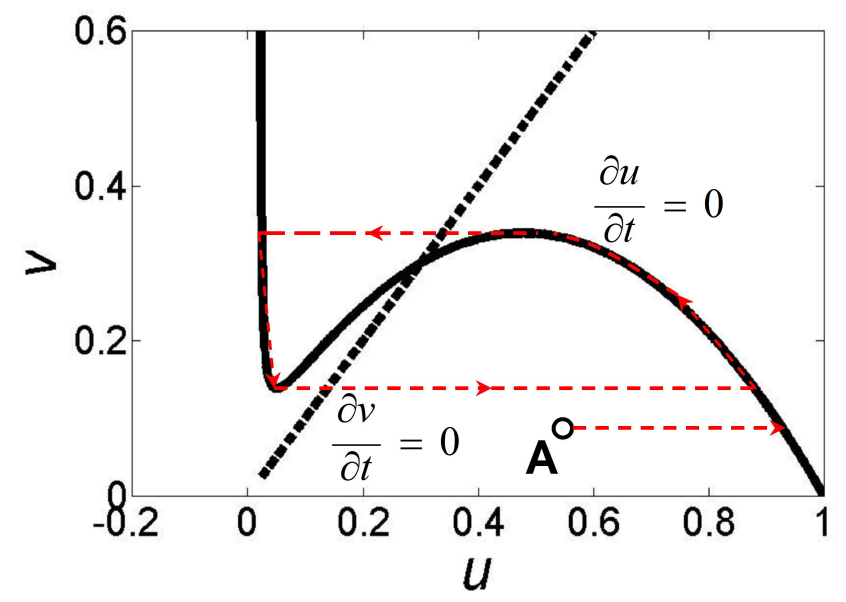

(b)

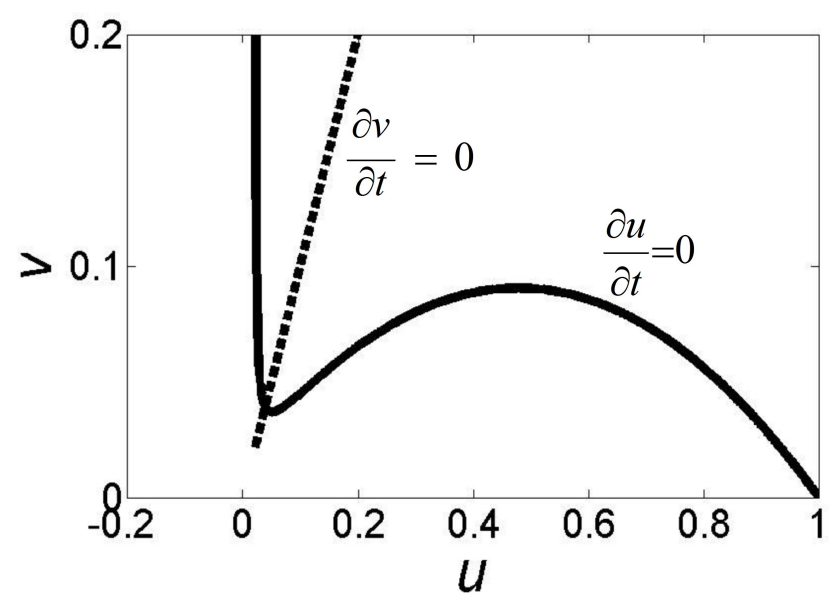

(c)

Figure 1.1. Three states described by the $u-v$ phase plane from the two-variable Oregonator model with $q=0.02$ and different $f$ values. (a) The excitable state with $f=0.25$. (b) The oscillatory state with $f=0.8$. A (o) is an arbitrary initial point. After a small perturbation, the system first approaches the $u$-nullcline branch and moves along the curve until it jumps horizontally to the other branch, closing the loop. (c) The excitable state with $f=3[4]$. 


\subsection{Effect of Light on the BZ Reaction}

The first studies on the effect of light on the temporal oscillations of the BZ reaction were in 1968, when Vavilin et al. [12] first reported that irradiation of ultraviolet (UV) light completely inhibited or strongly modified the oscillations in the cerium-catalyzed system. In 1973, Busse and Hess [13] first demonstrated the initialization of a propagating wave by UV radiation in a thin layer of cerium-ferroin catalyzed BZ solution. Gáspár et al. [14] in 1983 studied the influence of visible light on BZ oscillating systems using different catalysts in a batch reactor. They found that light has no effect on the $\mathrm{Ce}^{4+}$ system; however, the oscillating systems using the complexes $\mathrm{Fe}(\mathrm{phen}){ }_{3}^{2+}$ or $\mathrm{Ru}(\mathrm{bpy}){ }_{3}^{2+}$ as the catalyst were affected. Not only were the amplitude and frequency of oscillations changed by the illumination, but also the initiation and inhibition of oscillations were observed as a response to the illumination. In 1987, Bodet et al. [15] studied inhomogeneous perturbations of the BZ reaction catalyzed by ferroin with a focused laser beam and observed phase waves in the oscillating medium. Kuhnert [16] studied the transformation of phase waves into trigger waves by illuminating the $\mathrm{Ru}(\mathrm{bpy})_{3}^{2+}$ catalyzed $\mathrm{BZ}$ reaction system. In 1992, Weigt [17] reported chemic luminescence oscillations in the $\mathrm{Ru}(\mathrm{bpy})_{3}^{2+}$ catalyzed $\mathrm{BZ}$ reaction in a flowthrough reactor, with three separate emission peaks.

In the photosensitive BZ reaction, the currently widely used catalyst is tris(2,2'-bipyridine) ruthenium(II), $\mathrm{Ru}(\mathrm{bpy})_{3}^{2+}$, which was first adapted as a luminescent indicator for the demonstration of the BZ oscillatory reaction by Demas and Diemente in 1973 [18]. The ruthenium catalyst is sensitive to visible light and is photochemically excited by $452 \mathrm{~nm}$ light. The excited state of the catalyst then reacts with bromomalonic acid to produce bromide ions [19], an inhibitor of autocatalysis, in a series of reactions. 


\subsection{Modified Oregonator Model for the Photosensitive BZ}

\section{Reaction}

The BZ system with the $\mathrm{Ru}(\mathrm{bpy})_{3}^{2+}$ catalyst is sensitive to visible light, and excitability decreases with an increase in light intensity. The sensitivity of this system to light allows implementation of methods of spatiotemporal control based on illumination intensity.

The essential features of the system are described by a two-variable Oregonator model $[4,8]$ modified to include the photochemical pathway [9]:

$$
\begin{aligned}
& \frac{\partial u}{\partial t}=D_{u} \nabla^{2} u+\frac{1}{\epsilon}\left\{u-u^{2}-[\phi+f v] \frac{u-q}{u+q}\right\}, \\
& \frac{\partial v}{\partial t}=D_{v} \nabla^{2} v+u-v,
\end{aligned}
$$

where the dimensionless variables $u$ and $v$ correspond to $\left[\mathrm{HBrO}_{2}\right]$ and the $\mathrm{Ru}(\mathrm{III})$ catalyst concentration, respectively. The activator diffusion coefficient is $D_{u}$ while the corresponding diffusion term for $v$ is $D_{v}=0$, since the catalyst is immobilized in the gel. The kinetic parameters $\epsilon, q$ and $f$ are fixed. The parameter $\phi$ corresponds to the light intensity and controls the excitability of the medium [11].

Nullclines of $\frac{\partial u}{\partial t}=0$ and $\frac{\partial v}{\partial t}=0$ with different features can be obtained with different values of $\phi$ for a fixed value of $f$, as shown in Fig. 1.2. The excitability of the system can be adjusted through varying the light intensity $\phi$. In Fig. $1.2 \mathrm{a}$ and $1.2 \mathrm{~b}$, the intersection is on the middle branch of the $u$-nullcline with low $\phi$ values, generating an unstable steady state and thus the onset of oscillatory behavior. In Fig. 1.2c and 1.2d, high $\phi$ values give intersection points lying on the left branch of the $u$-nullcline, resulting in steady state behavior. It also can change the excitability of the system, with the larger the value of $\phi$, the lower the excitability. In the limit $\phi \rightarrow 0$, the reduced Oregonator model with two variables is the same as the original Oregonator model, proposed by 


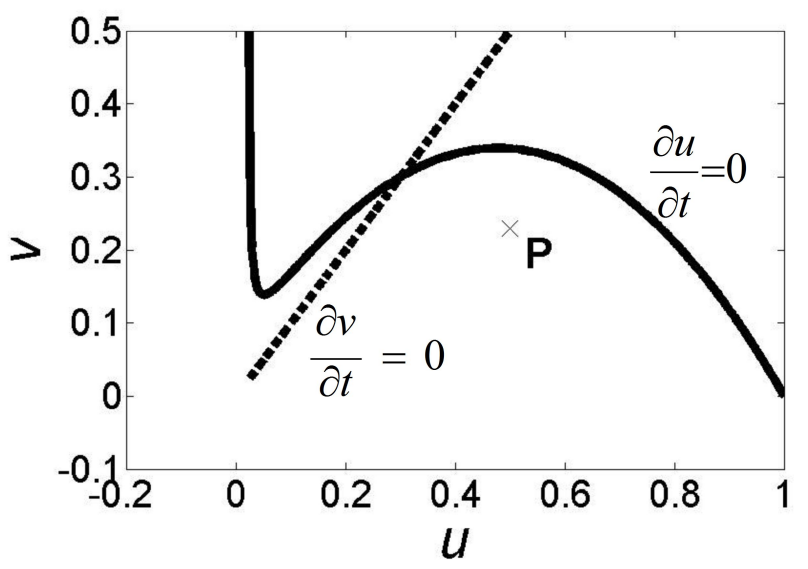

(a)

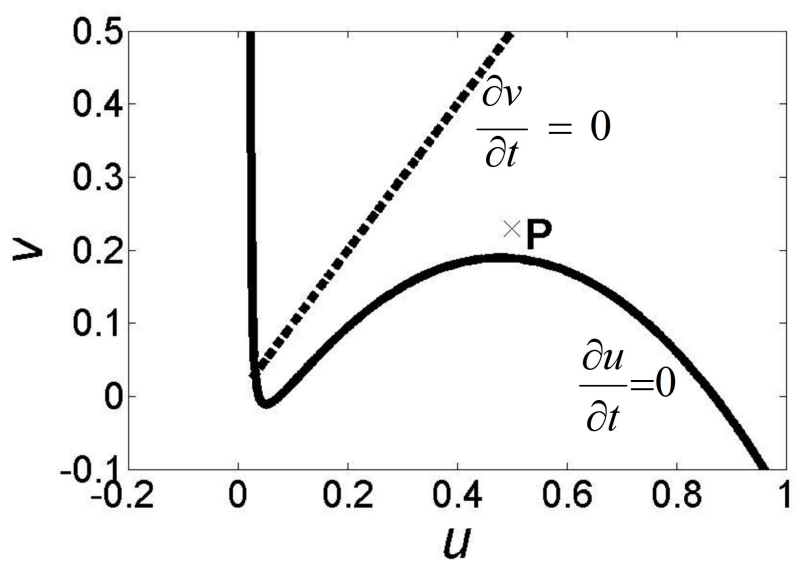

(c)

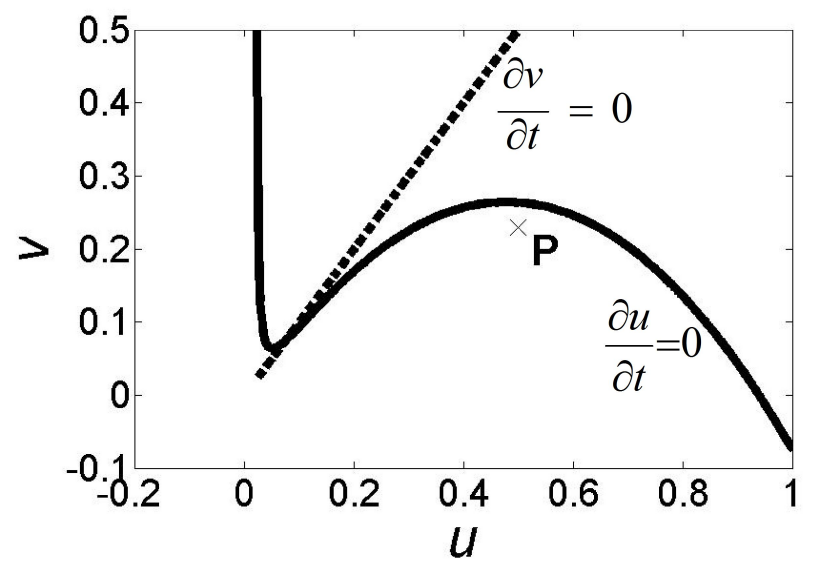

(b)

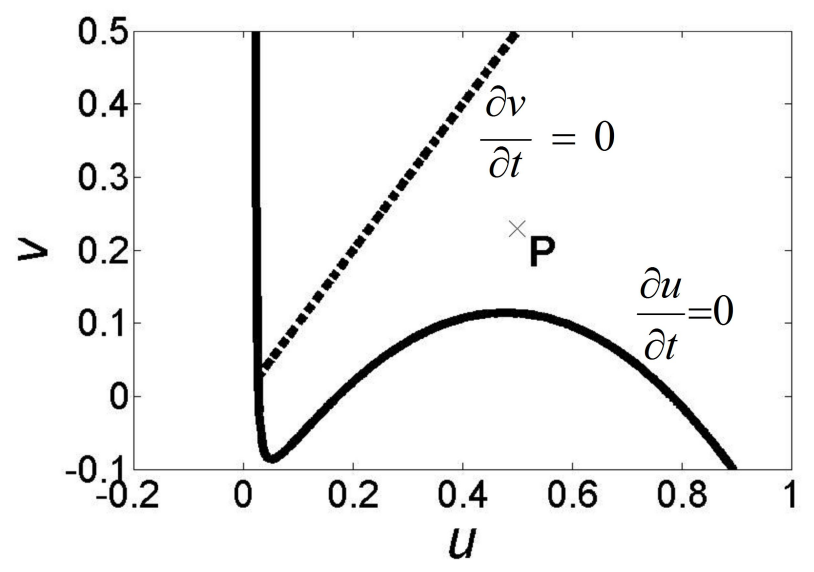

(d)

Figure 1.2. The effect of light intensity on the excitability of the photosensitive BZ reaction. The nullclines of $\frac{\partial u}{\partial t}=0$ and $\frac{\partial v}{\partial t}=0$ are plotted for Eqs. 1.5 with the parameters $q=0.02, f=$ 0.8 and different $\phi$ values. (a) $\phi=0$, (b) $\phi=0.06$, (c) $\phi=0.12$, and (d) $\phi=0.18$. An initial condition shown by the point $\mathrm{P}(\mathrm{x})$ is above the threshold for $\phi=0.0$ and $\phi=0.06$ but is below threshold for $\phi=0.12$ and $\phi=0.18$. 
the Field and Noyes in 1974 [20].

\subsection{Pattern Formation in the BZ Reaction}

\subsubsection{Patterns without External Forcing}

In the BZ reaction, the reactants undergo only a slight depletion during an oscillation excursion. Thus, the propagating pulse effectively regenerates the original kinetic state, allowing for the creation of chemical waves. In a 1D system, waves of excitation travel as a train of impulses. In 2D excitable media, the typical waves are spreading homocentric circular waves, also known as target patterns, and rotating spiral waves. Spreading homocentric spherical waves and rotating scroll waves are exhibited in 3D systems.

The $1 \mathrm{D}$ waves in the BZ reactions are formed in a confined space, as in a narrow capillary tube, and consist of a train of fronts following each other at a distance. Measurements in such simple geometries can be performed, for instance, to study the effect of an externally applied electrical field on wave properties [21].

In a $2 \mathrm{D}$ configuration, chemical waves $[22,23]$ in the $\mathrm{BZ}$ reaction were first reported by $\mathrm{A}$. N. Zaikin and A. M. Zhabotinsky [24, 25]. They studied trigger waves, where the autocatalytic kinetics of the reaction is coupled with diffusion, giving rise to propagating waves of chemical activity. The waves were found to propagate with constant velocities in the range of millimeters per minute.

The initiation of trigger waves depends crucially on triggering the reaction to the excited state; however, it also depends on the size of the excited region. There exists a critical nucleation size under which the outward diffusion of the autocatalyst is not able to support wave propagation $[26]$. 
Trigger waves in thin films of solution develop into target patterns and spiral waves [22, 27]. The chemical waves that constitute a target pattern emanate from a pacemaker center that determines the frequency of wave generation. When adjacent centers have different frequencies, the center with the higher frequency grows at the expense of the center of lower frequency. In time, the entire solution is entrained by the highest frequency pacemaker center and a single target pattern is exhibited. Different centers can initiate waves of different frequencies, and experimental evidence indicates that pacemaker centers are sites of heterogeneous catalysis [22, 27]. Sources of catalytic heterogeneities include particles of dust or scratches on the glass of the petri dish [28-30]. At such a site, the nonoscillatory reaction mixture is converted to a stable periodic oscillatory system. Further confirmation of the existence of heterogeneous catalytic sites comes from the experimental observation that filtering decreases the number of pacemaker centers, while adding dust increases the number of centers. Assuming the existence of heterogeneous catalytic sites, Tyson and Fife [8] developed a theoretical description based on a modified Oregonator model, which accounted for the essential features of target patterns [31].

Rotating spiral waves were first observed by Winfree [32] in the nonoscillatory excitable BZ system. While initial studies examined single armed spirals, subsequent work by Agladze and Krinsky [33] showed the existence of multi-armed spiral waves. Spiral waves are generated by shearing one or more waves in a target pattern. One convenient method of starting spiral waves is to tilt the dish containing the thin film of reaction mixture [32, 34], which results in the formation of spiral waves with opposite rotations at the ends of the unconnected segments.

If thick films of reaction mixture are used, the chemical wave can no longer be described in terms of two spatial dimensions. In a 3D medium, a spiral wave becomes a scroll wave $[22,27]$. The tip of the spiral becomes a filament which acts as the source for the scroll waves. Using topological arguments, Winfree $[27,35]$ proposed that the filament has a closed ring structure. It is therefore 
possible that very complex structures may form as the scroll waves propagate from the filament. This view of $3 \mathrm{D}$ scroll waves has been supported by experimental measurements [27, 34, 36]. Winfree and Strogatz have also suggested that exotic structures will arise from twisted [37] and knotted [38] filaments. Target patterns become propagating concentric spherical waves in a 3D medium [31].

During the 1990s, many more complex spatiotemporal patterns were observed in the $\mathrm{Ru}(\mathrm{bpy})_{3}^{2+}$ catalyzed BZ reaction. Jinguji et al. [39] studied the photo-induced waves in square, triangular, star and circular shapes. Waves were initiated at the boundaries of the corresponding opaque masks and the wave fronts propagated inward into the illuminated field, contrary to the usual direction of wave motion. Müller and co-workers [40, 41] used an argon laser beam to produce an unexcitable disk in a BZ medium, which erased the core structure of two adjacent spirals, generating an autonomous pacemaker for target patterns [40], and gave rise to multi-armed spirals [41] with spiral tips at equal distances around the disk boundary. Wave splitting following a short, high-intensity inhibitory light pulse was observed by Krug et al. [42] and Muñuzuri et al. [43].

\subsubsection{Patterns with External Forcing}

The photosensitive BZ reaction is the most widely studied chemical laboratory system for the dynamics of chemical waves, as the excitability of the medium can be efficiently controlled by either globally or locally altering the illumination and forcing frequency. The experimental spatiotemporal system can be prepared with the $\mathrm{Ru}(\mathrm{bpy})_{3}^{2+}$ catalyst immobilized in an external medium, such as silica gel or acrylamide, which is bathed in a continuously refreshed catalyst-free solution. A video projector and a video camera controlled by a computer allow real-time feedback for perturbing the light sensitive reaction and monitoring its response.

New kinds of pattern formations have been found in the photosensitive BZ reaction with exter- 
nal forcing. Petrov et al. [44] reported that a spiral wave transforms into a labyrinthine standing wave with periodic optical forcing and they found a sequence of frequency-locked resonant patterns as the forcing frequency was varied. Vanag et al. [45] reported oscillatory cluster patterns generated by global feedback.

Kádár et al. [46] reported stochastic resonance in a subexcitable medium, where wave propagation was made possible by imposing spatiotemporal noise on a medium unable to support sustained wave propagation. A negative-feedback control algorithm was used to stabilize the propagating wave segments [47]. Wave propagation in intricate patterns controlled by feedback-regulated excitability gradients in excitable media has been reported [48], which may one day lead to controlling abnormal electrical waves in the heart or brain to ward off a heart attack or epileptic seizure.

The impact of external forcing from homogeneous illumination on spiral waves have been studied. A number of behaviors were observed, including synchronization of the movement of the spiral tip with the external frequency, resonance drift of the spiral core, and irregular motion of the tip [49-52]. Observation of a spiral wave perturbed by a sequence of short light pulses revealed two new dynamic regimes for spiral tip behavior, entrainment and resonance attractors [53-56].

\subsubsection{Effect on Dynamics of Trigger Waves}

The velocity of a trigger wave is a characteristic property of a reaction-diffusion system. Its systematic study provides a key to understanding the properties of the system. The velocity of trigger wave propagation increases with increasing concentration of $\mathrm{Ru}(\mathrm{bpy})_{3}^{2+}$ and is proportional to the square root of the product of the concentrations of $\mathrm{H}_{2} \mathrm{SO}_{4}$ and $\mathrm{NaBrO}_{3}$. This was first shown by Field and Noyes for the ferroin catalyzed system [20]. Kuhnert and Krug [57] first quantitatively

studied the wave propagation in the non-illuminated $\mathrm{Ru}(\mathrm{bpy})_{3}^{2+}$ catalyzed systems and compared the results with the ferroin system [20]. 
Light was found to decrease the velocity and even completely inhibit the wave propagation for the concentration conditions studied. Reddy et al. [58] systematically studied the influence of visible light on wave velocities in the $\mathrm{Ru}(\mathrm{bpy})_{3}^{2+}$ catalyzed $\mathrm{BZ}$ reaction in a thin film of solution. Krug et al. [42] studied the tris(4,4'-dimethyl-2,2'-bipyridyl) ruthenium(II) catalyzed BZ system in a continuously fed gel reactor and also found that trigger wave velocity decreased with increasing light intensity until the waves were completely extinguished.

The excitability of the medium affects not only the velocity of the wave but also the other characteristics of wave propagation. Sendiña-Nadal et al. [59] studied wave propagation in a medium with disordered excitability. They found that wave speed in one dimension was smaller than that corresponding to a homogeneous medium, while in two dimensions, wave velocity increased due to the roughening of the front. Agladze et al. [60] studied the propagation of chemical waves in a photosensitive BZ system at the boundary of excitable and inhibitory fields. Depending on the degree of excitability of the two areas, waves were found to either penetrate into the inhibitory region or collapse in the excitable zone. 


\section{Reference}

[1] A. M. Zhabotinsky, "A history of chemical oscillations and waves," Chaos 1, 379-386 (1991). Online Version 1

[2] R. Kapral and K. Showalter, Chemical waves and patterns (Kluwer Academic Publishers, Boston, MA, 1995).

Online Version 1

[3] R. J. Field, E. Körös, and R. M. Noyes, "Oscillations in chemical systems. II. Thorough analysis of temporal oscillation in the bromate-cerium-malonic acid system," J. Am. Chem. Soc. 94, 8649-8664 (1972).

Online Version 2

[4] R. J. Field and R. M. Noyes, "Oscillations in chemical systems. IV. Limit cycle behavior in a model of a real chemical reaction," J. Chem. Phys. 60, 1877-1884 (1974).

Online Version 2, 4, 5, 7

[5] R. Field and M. Burger, Oscillations and traveling waves in chemical systems (John Wiley \& Sons, Princeton, NJ, 1985).

Online Version 2

[6] I. R. Epstein and K. Showalter, "Nonlinear chemical dynamics: Oscillations, patterns, and chaos," J. Phys. Chem. 100, 13132-13147 (1996).

Online Version 3

[7] A. S. Mikhailov and K. Showalter, "Control of waves, patterns and turbulence in chemical systems," Phys. Rep. 425, 79-194 (2006).

Online Version 3 
[8] J. J. Tyson and P. Fife, "Target patterns in a realistic model of the Belousov-Zhabotinsky reaction," J. Chem. Phys. 73, 2224-2237 (1980).

Online Version 3, 4, 7, 10

[9] H. J. Krug, L. Pohlmann, and L. Kuhnert, "Analysis of the modified complete Oregonator accounting for oxygen sensitivity and photosensitivity of Belousov-Zhabotinskii systems," J. Phys. Chem. 94, 4862-4866 (1990).

Online Version 3, 4, 7

[10] J. J. Tyson, "Scaling and reducing the Field-Körös-Noyes mechanism of the BelousovZhabotinsky reaction," J. Chem. Phys. 86, 3006-3012 (1982).

Online Version 4

[11] F. Chirila, Feedback control of wave propagation patterns in excitable media, Ph.D. thesis, West Virginia University (2003). 4, 7

[12] V. A. Vavilin, A. M. Zhabotinsky, and A. N. Zaikin, "Effect of ultraviolet radiation on the self-oscillatory oxidation of malonic acid derivatives," Russ. J. Phys. Chem. 42, 3091-3094 (1968). 6

[13] H. Busse and B. Hess, "Information transmission in a diffusion-coupled oscillatory chemical system," Nature 244, 203-205 (1973).

Online Version 6

[14] V. Gáspár, G. Bazas, and M. T. Beck, "The influence of visible light on the BelousovZhabotinskii oscillating reactions applying different catalysts," Z. Phys. Chem. (Leipzig) 264, 43-48 (1983).

Online Version 6 
[15] J. M. Bodet, J. Ross, and C. Vidal, "Experiments on phase diffusion waves," J. Chem. Phys. 86, 4418-4424 (1987).

Online Version 6

[16] L. Kuhnert, "Photochemische manipulation von chemischen wellen," Naturwiss. 73, 96-97 (1986).

Online Version 6

[17] H. R. Weigt, "Chemiluminescence oscillations driven by a flow-through reactor in the $\left[\mathrm{Ru}(\mathrm{bpy})_{3}\right]^{2+}$ catalyzed Belousov-Zhabotinskii reaction," Angew. Chem. Int. Ed. Engl. 31, 355-357 (1992).

Online Version 6

[18] J. N. Demas and D. J. Diemente, "An oscillating chemical reaction with a luminescent indicator," J. Chem. Ed. 50, 357-358 (1973).

Online Version 6

[19] S. Kádár, T. Amemiya, and K. Showalter, "Reaction mechanism for light sensitivity of the $\mathrm{Ru}(\mathrm{bpy})_{3}^{2+}$ catalyzed Belousov-Zhabotinsky reaction," J. Phys. Chem. A 101, 8200-8206 (1997).

Online Version 6

[20] R. J. Field and R. M. Noyes, "Oscillations in chemical systems. V. Quantitative explanation of band migration in the Belousov-Zhabotinskii reaction,” J. Am. Chem. Soc. 96, 2001-2006 (1974).

Online Version 9, 12 
[21] J. Ross, S. C. Müller, and C. Vidal, "Chemical waves," Science 240, 460-465 (1988). Online Version 9

[22] A. T. Winfree, "Two kinds of wave in an oscillating chemical solution," Faraday Symp. Chem. Soc. 9, 38-46 (1974).

Online Version 9, 10

[23] E. J. Reusser and R. J. Field, "The transition from phase waves to trigger waves in a model of the Zhabotinskii reaction," J. Am. Chem. Soc. 101, 1063-1071 (1979).

Online Version 9

[24] A. N. Zaikin and A. M. Zhabotinsky, "Concentration wave propagation in two-dimensional liquid-phase self-oscillating system," Nature 225, 535-537 (1970).

Online Version 9

[25] A. M. Zhabotinsky and A. N. Zaikin, "Autowave processes in a distributed chemical system," J. Theor. Biol. 40, 45-61 (1973).

Online Version 9

[26] Á. Tóth, Chemical Waves: The Effect of Geometrical Constraints, Ph.D. thesis, West Virginia University (1994). 9

[27] A. Winfree, The geometry of biological time (Springer-Verlag, New York, 2000). Online Version 10, 11

[28] E. Mori and J. Ross, "Origin of spontaneous wave generation in excitable chemical systems," J. Phys. Chem. 96, 8053-8060 (1992).

Online Version 10 
[29] J. J. Tyson, "Singular perturbation theory of target patterns in the Belousov-Zhabotinskii reaction," J. Chim. Physique 84, 1359-1365 (1987).

Online Version

[30] H. Nagashima, "Target patterns and pacemakers in a reaction-diffusion system," J. Phys. Soc. Jpn. 60, 2797-2799 (1991).

Online Version 10

[31] J. S. Reckley, Studies of Nonlinear Dynamics in Chemical Systems, Ph.D. thesis, West Virginia University (1988). 10, 11

[32] A. T. Winfree, "Spiral waves of chemical activity," Science 175, 634-636 (1972).

Online Version 10

[33] K. I. Agladze and V. I. Krinsky, "Multi-armed vortices in an active chemical medium," Nature 296, 424-426 (1982).

Online Version 10

[34] A. T. Winfree, "Rotating chemical reactions," Sci. Am. 230, 82-95 (1974). Online Version 10, 11

[35] A. T. Winfree and S. H. Strogatz, "Singular filaments organize chemical waves in three dimensions: I. Geometrically simple waves," Physica D 8, 35-49 (1983). Online Version 10

[36] B. J. Welsh, J. Gomatam, and A. E. Burgess, "Three-dimensional chemical waves in the Belousov-Zhabotinskii reaction," Nature 304, 611-614 (1983).

Online Version 11 
[37] A. T. Winfree and S. H. Strogatz, "Singular filaments organize chemical waves in three dimensions II. Twisted waves," Physica D 9, 65-80 (1983). Online Version 11

[38] P. De Kepper, I. R. Epstein, and K. Kustin, "Bistability in the oxidation of arsenite by iodate in a stirred flow reactor," J. Am. Chem. Soc. 103, 6121-6127 (1981). Online Version 11

[39] M. Jinguji, M. Ishihara, and T. Nakazawa, "Photoinduced formation of spatial patterns in the Belousov-Zhabotinsky reaction," J. Phys. Chem. 94, 1226-1229 (1990). Online Version 11

[40] S. C. Müller, O. Steinbock, and J. Schütze, "Autonomous pacemaker of chemical waves created by spiral annihilation," Physica A 188, 47-54 (1992).

Online Version 11

[41] O. Steinbock and S. Müller, "Multi-armed spirals in a light-controlled excitable reaction," Int. J. Bifur. Chaos 3, 437-443 (1993).

Online Version 11

[42] H.-J. Krug, H. Brandtstaedter, and L. Pohlmann, "Nucleation and wave propagation in the modified Oregonator and comparison with experiments in a photosensitive BelousovZhabotinsky gel reactor," J. Phys. Chem. 99, 10237-10245 (1995).

Online Version 11, 13

[43] A. P. Mun̈uzuri, V. Pérez-Villar, and M. Markus, "Splitting of autowaves in an active medium," Phys. Rev. Lett. 79, 1941-1944 (1997).

Online Version 11 
[44] V. Petrov, Q. Ouyang, and H. L. Swinney, "Resonant pattern formation in a chemical system," Nature 388, 655-657 (1997).

Online Version 12

[45] V. K. Vanag, L. Yang, M. Dolnik, A. M. Zhabotinsky, and I. R. Epstein, "Oscillatory cluster patterns in a homogeneous chemical system with global feedback," Nature 406, 389-391 $(2000)$.

Online Version 12

[46] S. Kádár, J. Wang, and K. Showalter, "Noise-supported traveling waves in sub-excitable media," Nature 391, 770-772 (1998).

Online Version 12

[47] E. Mihaliuk, T. Sakurai, F. Chirila, and K. Showalter, "Experimental and theoretical studies of feedback stabilization of propagating wave segments," Faraday Discuss. Chem. Soc. 120, 383-394 (2002).

Online Version 12

[48] T. Sakurai, E. Mihaliuk, F. Chirila, and K. Showalter, "Design and control of wave propagation patterns in excitable media," Science 296, 2009-2012 (2002).

Online Version 12

[49] M. Braune and H. Engel, "Compound rotation of spiral waves in active media with periodically modulated excitability," Chem. Phys. Lett. 211, 534-540 (1993).

Online Version 12

[50] M. Braune, A. Schrader, and H. Engel, "Entrainment and resonance of spiral waves in active 
media with periodically modulated excitability," Chem. Phys. Lett. 222, 358-362 (1994).

Online Version

[51] O. Steinbock, V. Zykov, and S. C. Müller, "Control of spiral-wave dynamics in active media by periodic modulationof excitability," Nature 366, 322-324 (1993).

Online Version

[52] V. S. Zykov, O. Steinbock, and S. C. Müller, "External forcing of spiral waves," Chaos 4, 509-518 (1994).

Online Version 12

[53] S. Grill, V. S. Zykov, and S. C. Müller, "Feedback-controlled dynamics of meandering spiral waves," Phys. Rev. Lett. 75, 3368-3371 (1995).

Online Version 12

[54] S. Grill, V. S. Zykov, and S. C. Müller, "Spiral wave dynamics under pulsatory modulation of excitability," J. Phys. Chem. 100, 19082-19088 (1996).

Online Version

[55] D. M. Goldschmidt, V. S. Zykov, and S. C. Müller, "Transition to irregular dynamics of spiral waves under two-channel feedback," Phys. Rev. Lett. 80, 5220-5223 (1998).

Online Version

[56] O.-U. Kheowan, V. S. Zykov, O. Rangsiman, and S. C. Müller, "Transitions between orbits of resonance attractors for spiral waves," Phys. Rev. Lett. 86, 2170-2173 (2001).

Online Version 12

[57] L. Kuhnert and H. J. Krug, "Kinetics of chemical waves in the acidic bromate-malonic acidtris(bipyridine)ruthenium $(2+)$ system in comparison with the ferroin system," J. Phys. Chem. 
91, 730-733 (1987).

Online Version 12

[58] M. K. R. Reddy, Z. Nagy-Ungvarai, and S. C. Müller, "Effect of visible light on wave propagation in the ruthenium-catalyzed Belousov-Zhabotinsky reaction," J. Phys. Chem. 98, 1225512259 (1994).

Online Version 13

[59] I. Sendiña-Nadal, A. P. Muñuzuri, D. Vives, V. Pérez-Muñuzuri, J. Casademunt, L. RamrezPiscina, J. M. Sancho, and F. Sagués, "Wave propagation in a medium with disordered excitability," Phys. Rev. Lett. 80, 5437-5440 (1998).

Online Version 13

[60] K. Agladze, Á. Tóth, T. Ichino, and K. Yoshikawa, "Propagation of chemical waves at the boundary of excitable and inhibitory fields," J. Phys. Chem. A 104, 6677-6680 (2000). Online Version 13 


\section{Chapter 2}

\section{Neural Network Model System}

Massive and hierarchical networking of the human brain seems to be the fundamental precondition for the emergence of consciousness and complex behavior [1]. The behavior of the individual elements in the biological neural network, neurons, are described in this Chapter. Inspired by the structure of the biological neural networks, artificial neural networks have been designed to solve a variety of problems in pattern recognition, prediction, optimization and control.

As the connections between the neurons, synapses in the biological neural network are widely believed to have a contribution in memory storage. In 1949, Donald Hebb firstly proposed a important viewpoint about the dynamics of synapses [2], but it also can explain the origin of memory storage. In past decades, an improved Hebbian rule, called spike-timing-dependent plasticity (STDP), has attracted much attention among biologists and neurologists [3].

Here, after introducing the fundamentals of networks and biological neural networks, we will focus on illustrating the principles of Hebb's rule and STDP. In Chapters 3, STDP will be applied in a BZ chemical network. 


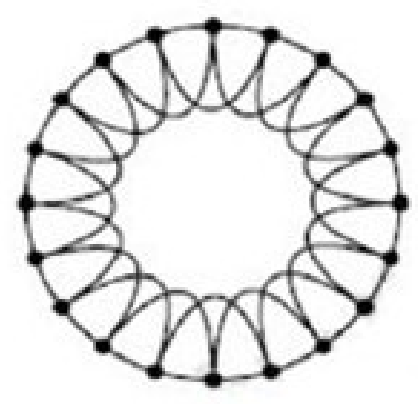

(a)

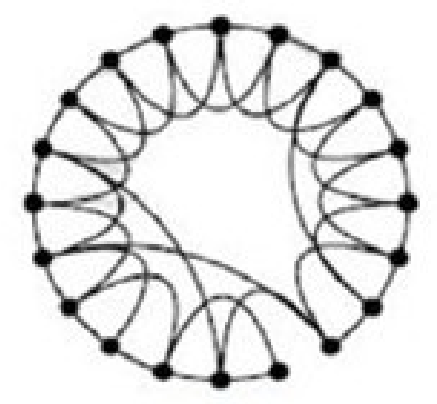

(b)

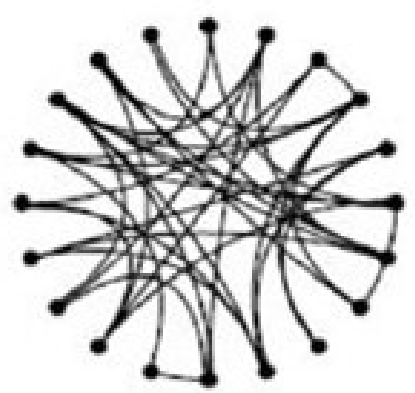

(c)

Figure 2.1. Three typical network structures with the number of nodes $n=20$. (a) Regular network. (b) Randomly rewiring of a small fraction of the links to new nodes produces a WattsStrogatz small-world network. (c) Random network. Figure from reference [6].

\section{$2.1 \quad$ Introduction}

\subsubsection{Concept of Network}

A network consists of a set of nodes with connections between them called links [4, 5]. These connections may be random or exist in certain relationships. Two important measures can be defined to characterize their structure in the network. One is the clustering coefficient $C$, which is the average fraction of a node's neighbors that are also neighbors of each other. If node $A$ has $z$ neighbors, there are at most $\frac{z(z-1)}{2}$ links between them. $C_{A}$ is the fraction of these links that actually exist. The clustering coefficient $C$ is the average value of $C_{A}$ over all nodes in the network. The second important measure is the average length $L$, which is the average distance of the shortest path between any two nodes in the network.

Figure 2.1 shows networks classified according to the two parameters. A regular network typically has high values of $C$ and $L$, indicating a high connectivity between neighboring nodes but large average separation between nodes. In contrast, a random network has low $C$ and $L$ 


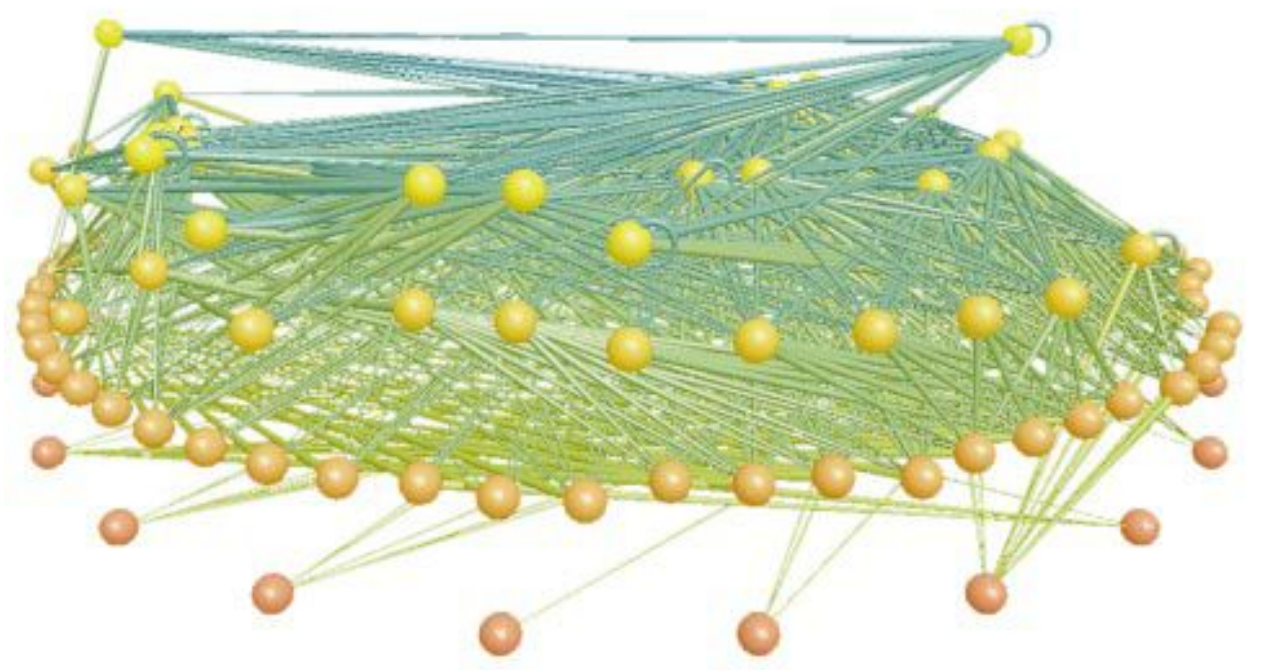

Figure 2.2. The food web in Little Rock Lake, Wisconsin. Nodes are functionally distinct 'trophic species' containing all taxa that share the same set of predators and prey. Height indicates trophic level with mostly phytoplankton at the bottom and fishes at the top. Figure from reference [7].

values, indicating a low connectivity between neighboring nodes and small average separation between nodes [5]. A small-world network has the important property of both a high clustering coefficient $C$ and a low average distance $L[6,7]$.

\subsubsection{Natural Networks}

There are many examples of networks in nature. Most natural networks display substantial nontrivial topological features, with patterns of connection between their elements that are neither purely regular nor purely random. Therefore, they can not be simply classified as one of the networks in Fig. 2.1 and are often referred to as complex networks [4].

The study of such complex networks has received great attention in recent years [8]. Empirical 
studies have shed light on the topology of food webs, electrical power grids, cellular and metabolic networks, the World-Wide Web (WWW), the Internet backbone, and the neural network of the nematode worm Caenorhabditis elegans [7]. Examples of various food webs have provided clues into ecosystem organization and its relationship with different types of ecological stability [9]. Figure 2.2 shows the currently largest food web in Little Rock Lake, Wisconsin [7, 10]. The wiring network structure is an intricate tangle; cannibalism is shown with self-loops and omnivory (feeding on more than one trophic level) is shown by different coloured links to the consumers.

Another example is the WWW, where nodes are web pages connected by the Internet, made up of millions of hosts. Loaded pages and links are created and lost every minute, dynamically changing the complexity of the WWW system. Since modern society depends strongly on this infrastructure, it is very important to have detailed information about it in order to be able to predict its behavior or take the correct actions during a crisis [11, 12].

\subsection{Biological Neural Networks}

\subsubsection{Neurons and Spikes}

Neural networks are a class of biological networks of considerable importance [4]. Neurons are the individual elements in neural networks. In the human brain, there exists about 10 billion neurons, each connected to many other neurons to form a complex network structure with various functions including pattern reorganization and memory storage [14].

A neuron is generally covered with a semi-permeable membrane, $5 \mathrm{~nm}$ in thickness, which can selectively absorb and reject ions (e.g., $\mathrm{Na}^{+}$and $\mathrm{K}^{+}$) found in the intracellular fluid, which maintains an equilibrium polarized condition with a certain resting potential. A diagram of a typical motor neuron is shown in Fig. 2.3. It has a roughly spherical cell body called the soma 


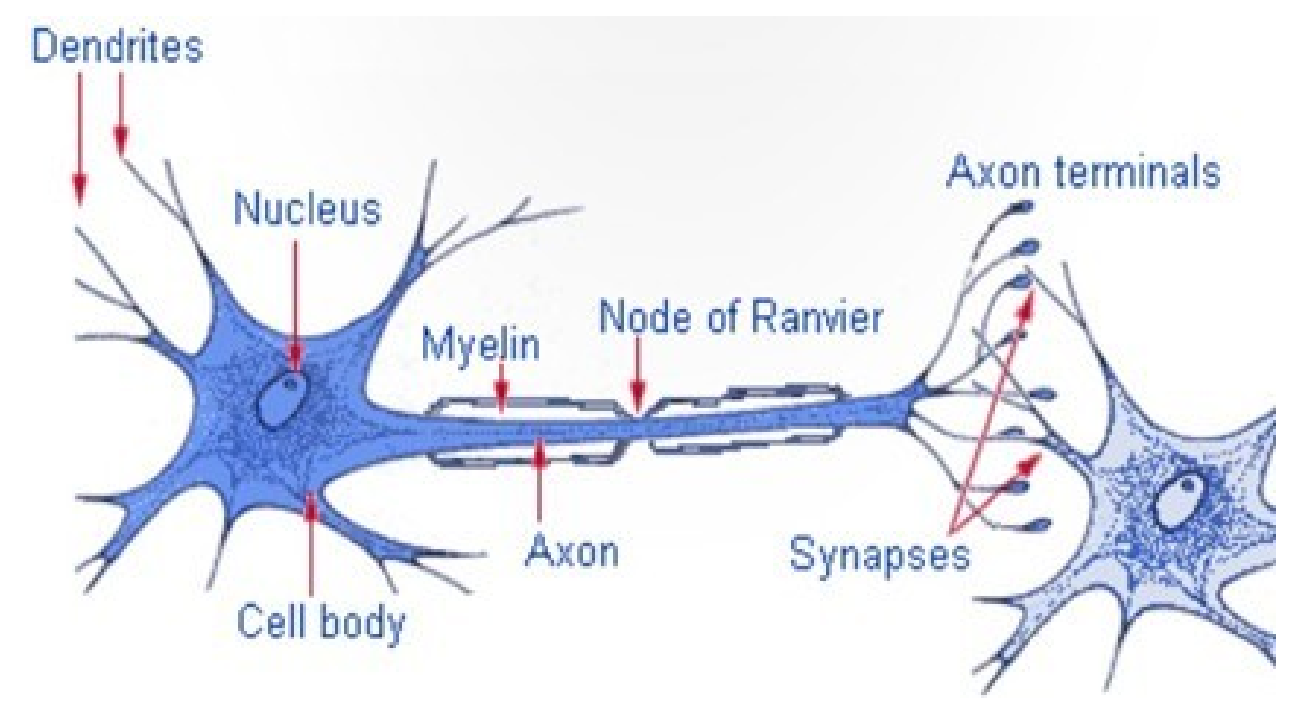

Figure 2.3. Diagram of a typical motor neuron. Figure from reference [13].

and extensions called dendrites. A specific extension called the axon or nerve fibre, with a variant length from a fraction of a millimeter to a meter, connects a neuron to other neurons. Myelin with periodic gap, node of ranvier, is a dielectric (electrically insulating) material that forms a layer around the axon. At the other end, an axon is separated into several branches to form the synapses, responsible for transmitting signals from one neuron to another one [15].

The soma may generate signals (nerve impulses) and transmit them to other neurons. A neuron receives signals from a large number of other neurons via its synaptic connections, causing either a decrease or an increase in its soma potential. If the synapses cause depolarization, they are called excitatory synapses; otherwise, they are called inhibitory synapses. If nerve impulses through other synapses arrive at the same time, the neuron may fire, where the internal potential becomes positive and a reversal in the potential occurs in the axon, as shown in Fig. 2.4. This is called the action potential or spike [17], which results in an electric current flowing from the region at the action potential to an adjacent region on the axon with the resting potential. Because the depolarized parts of the neuron can not immediately become active again, it is incapable of being 


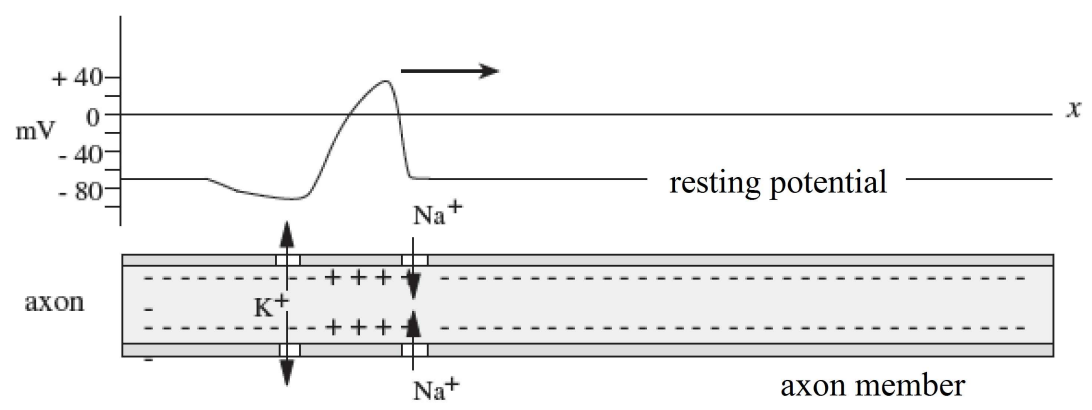

(a)

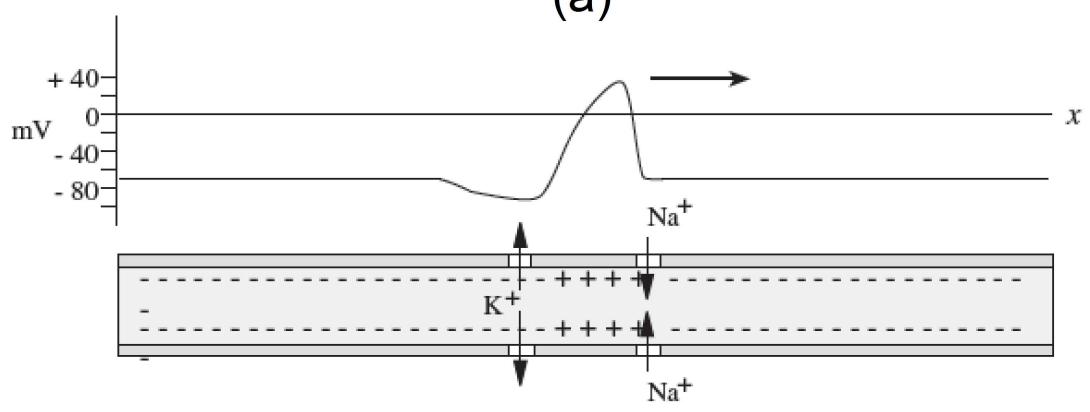

(b)

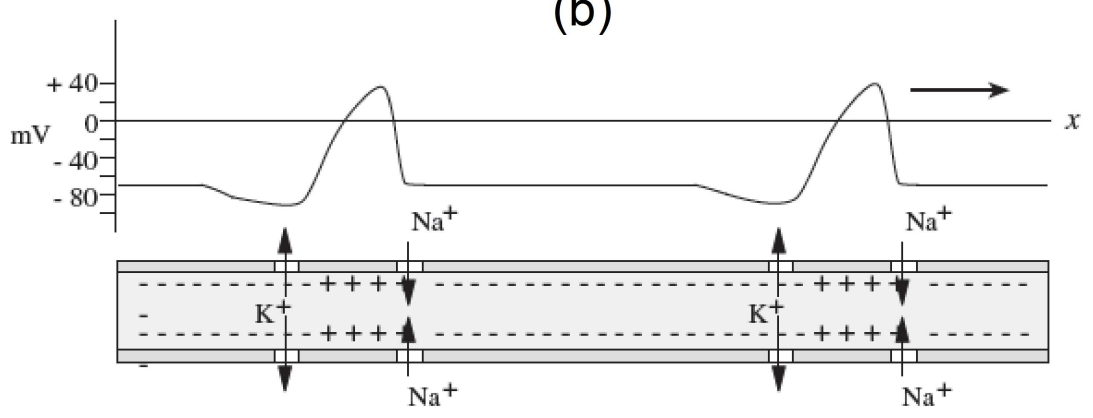

(c)

Figure 2.4. A schematic diagram of typical neuron spikes. (a) The action potential on the axon;

(b) transmission of an action potential along the axon; (c) the action potential trains with the refractory period. Figure from reference [16]. 


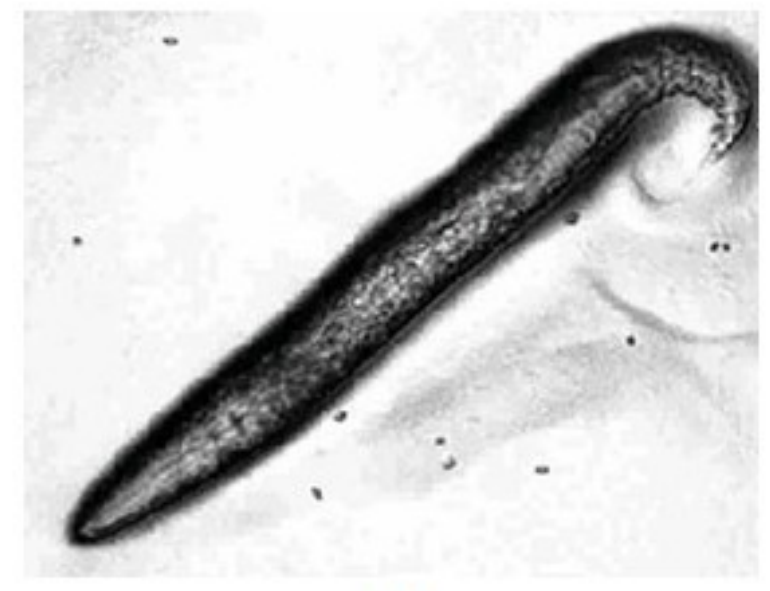

(a)

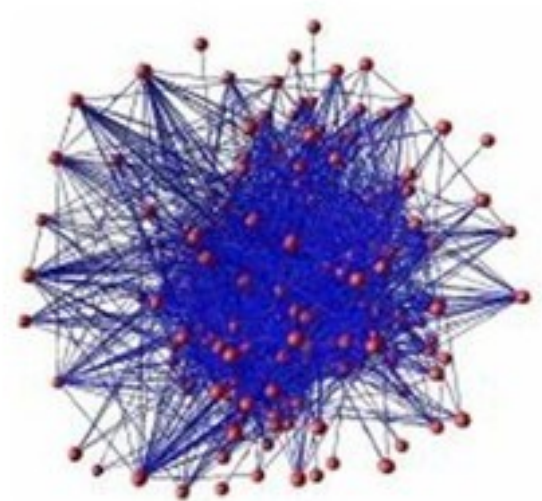

(b)

Figure 2.5. (a) A magnified picture of $C$. elegans (actual length is approximately $1 \mathrm{~mm}$ ). Figure from reference [19]. (b) A simple model to illustrate the C. elegans neural network. Red nodes represent neurons and links show the connections between them. Spatial positions are randomly placed. Figure from reference [20].

reexcited for a period of time called the refractory period. The refractory period is about 1 ms; therefore, a neuron can typically fire and generate action potentials at a rate of up to 1000 pulses per second with approximately constant amplitudes. Each neural spike is an all-or-none, selfpropagating regenerative event, as each signal has the same form and amplitude. However, the number of pulses and the time interval between pulses are controlled by the statistics associated with the arrival of excitatory inputs at the neuron's synaptic junctions [18]. At any time, many neurons are firing; even when asleep, $5 \times 10^{7}$ nerve impulses per second are being relayed between the brain and other parts of the body. This rate significantly increases when awake [14].

The study of the structure and function of biological neural networks is still in its infancy $[21,22]$. Biological neurons self-assemble to form complicated neural networks. Although the network structure of the brain at larger scales has been extensively investigated [4, 23, 24], the 
detailed topology of real neural networks is extremely difficult to measure. One example where reconstruction has been achieved is the neural network of a small worm called Caenorhabditis elegans by White et al., shown in Fig. 2.5 [19, 25]. Owing to its simple nervous system, all the connections of this network have been elucidated [26]. Treating each neuron as a node and each connection as a link, the nervous system of $C$. elegans consists of 282 nodes and 2,335 unique links. Panel (b) of Fig. 2.5 shows the actual neural network of C. elegans [20].

\subsubsection{Hebbian Learning}

In a neural network system, each neuron is connected to many other neurons by synapses, which act as links. The synaptic inputs may be excitatory or inhibitory to the neuron. Excitatory links increase the activity of the neuron, while inhibitory links suppress the activity of the neuron. The synaptic weight (usually denoted by $w$ ) is used to describe the strength and type of connection between two neurons. If $w>0$, the synaptic connection is an excitatory link; if $w<0$, the synaptic link is inhibitory.

In 1949, Donald Hebb first proposed the idea of connecting neural activity with synaptic plasticity, which is now called Hebbian learning or Hebb's rule [2]: "When an axon of cell A is near enough to excite a cell B and repeatedly or persistently takes part in firing it, some growth process or metabolic change takes place in one or both cells such that A's efficiency, as one of the cells firing B, is increased."

Hebb's rule provides a simple physiology-based model to mimic the activity dependent features of synaptic plasticity. Hebbian learning can be summarized in a simple statement: if neurons A and $\mathrm{B}$ are activated simultaneously or within a narrow time window of each other, the synapse between them is strengthened. An algorithm in Hebb's rule is used to update the weight of the neuronal connections within the neural network. In the classical Hebb's rule, there is no algorithm 
for synapses to become weaker and there is no upper bound that limits how strong the connectivity can become [27]. Therefore, the rule gives rise to dynamical behavior that is intrinsically unstable.

Evidence for the Hebbian rule was discovered in 1973 with the observation of persistent enhancement of synaptic transmission by tetanic stimulation in the hippo-campus, a phenomenon now generally referred to as long-term potentiation (LTP) [28]. Two features of LTP, the associativity and input specificity, match the properties of some forms of learning and memory, suggesting that LTP may underlie these cognitive functions [29]. However, both of synaptic strengthening or LTP and synaptic weakening, or long term depression (LTD), have been observed in experiments $[28,30]$, demonstrating deficiencies in Hebb's rule. Different versions of the Hebbian-like rules have been proposed to give more realistic behavior.

\subsubsection{Effects of Synaptic Connections on Memory Storage}

The changes of the synaptic connections between neurons are widely believed to be important for both memory storage and the activity dependent development of neuronal networks [3]. The Hebbian rule is often summarized as "Cells that fire together, wire together" to explain "associative learning" [2]. However, one key requirement of such changes in synaptic connections is that the inputs of a neuron can compete with the activity-dependent development, so that strengthening some inputs weakens others, and they should finally generate a stable distribution of synaptic weights [3].

An implementation of an unconstrained Hebbian rule does not produce a stable weight distribution. The initially strengthened synaptic weights will grow to infinitely large values because their correlation with the postsynaptic activity continues increasing. Some constraints can be introduced to remedy this problem, including a constant sum of all weights, regulating neuronal excitability by adjusting the threshold for potentiation, and scaling all of a neuron's synaptic 
weights. However, the choice of constraint strongly influences the neuron behavior of the Hebbian model [3].

\subsection{Artificial Neural Networks}

\subsubsection{Theoretical Models of Neurons}

Numerous models have been proposed to simulate the dynamics of individual idealized pointlike neurons at various levels of detail, including the Hodgkin-Huxley, Morris-LeCar, FitzHughNagumo, quadratic integrate-and-fire, and integrate-and-fire models [17]. Typically, neurons are seen as simple leaky capacitors with an internal battery to maintain them in a negative holding potential relative to the exterior. Both the excitatory and inhibitory synaptic inputs act to temporarily connect the capacitor to depolarizing or hyperpolarizing batteries. Neurons display approximately linear charging behavior as capacitors. Other models are simplified to resemble nonlinear oscillators, allowing rigorous phase-space analysis of the dynamics [12].

Generally, the depolarization from only a single synapse is not sufficient to fire a neuron in a biological neural network. In the integrate-and-fire model, the action potentials are generated by the following simple rule [31]: "An action potential occurs whenever the membrane potential reaches a threshold value $V_{t h}$. Immediately after that, the membrane potential is reset to a value $V_{\text {reset. }}$ " This means that when the combination of all the input signals is above a certain threshold for a neuron, the neuron fires and then relaxes to its rest state. Much effort has been spent on trying to experimentally determine the firing thresholds of neurons. The simple concept of spike threshold was shown in Fig. 2.6, although a clear-cut voltage value, above which the neuron fires but below which no spikes occur, may not exist in the biological neural system [16]. 


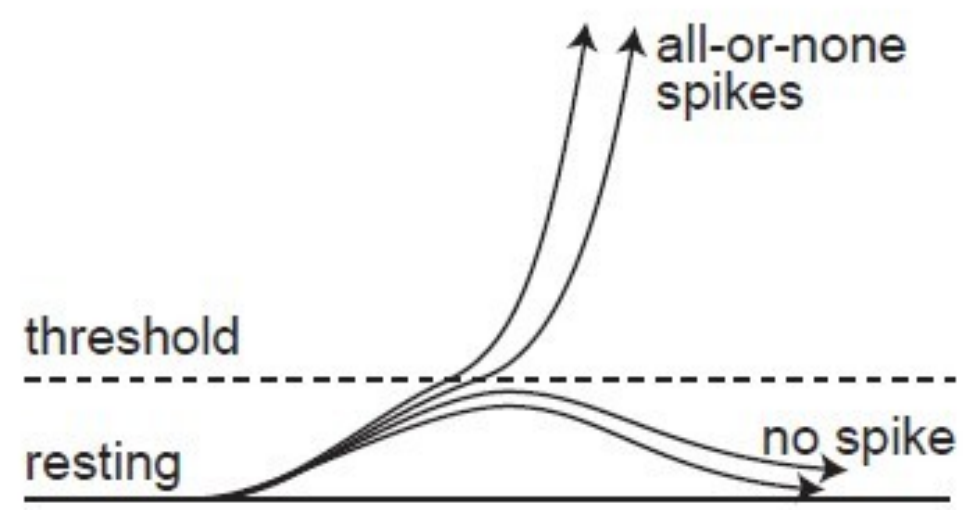

Figure 2.6. The concept of a firing threshold. Figure from reference [17].

\subsubsection{Network Models Inspired by Biological Neural Networks}

Artificial neural networks have been developed based on the structure of actual biological neural networks [32]. A network is created from a large number of simple processing units, each performing a weighted sum of its inputs and then firing a signal if the total input exceeds a certain threshold [33]. One approach to study such artificial neural networks is as follows: construct a general 'trainable' network with specific learning rules so that the weights of links between neurons can be changed to enable the network to perform goal-directed tasks.

Hebb's rule has been widely used in the area of artificial neural networks. For example, the Hopfield model is an associative memory model using Hebb's rule for all possible pairs of weights with binary units in the network. The state variable $x_{i}$ of the neuron $i$ takes on either one of the two possible values 1 or -1 , which corresponds to the firing state or not firing state, respectively. Another important artificial neural network is the back-propagation algorithm, as shown in Fig. 2.7, which is a method for training multilayer, feedforward networks with non-linear units to perform gradient descent on a suitably defined error function. However, these artificial neural networks ignore many fundamental details of biological neural networks. For example, the neurons 


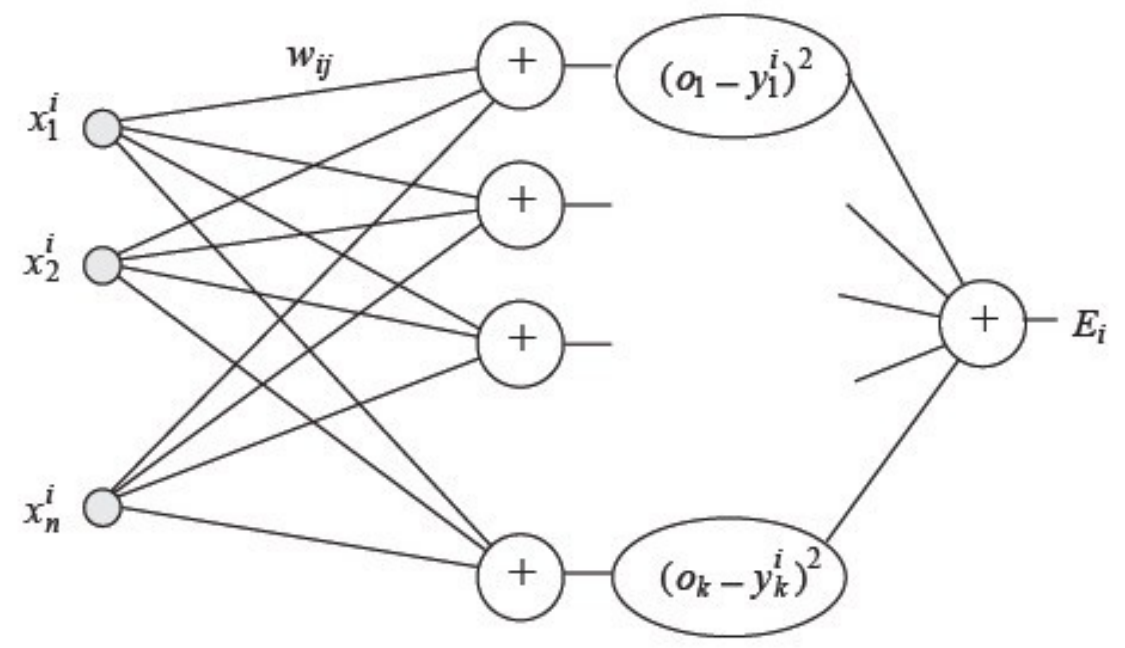

Figure 2.7. The back-propagation network for a linear associative memory. The units in the first layer are linear associators. The learning task consists in finding the weight matrix $\mathrm{W}$ with elements $w_{i j}$ that produces the best mapping of $o_{1}, o_{2}, \ldots, o_{m}$ from the input vectors $x_{1}, x_{2}, \ldots$ . , $x_{m}$ by comparing to the target vectors $y_{1}, y_{2}, \ldots, y_{m}$ with the computed quadratic error $E_{i}$. Figure from reference [16]. 
in the brain are extremely nonlinear with complex temporal dynamics and communicate with spikes rather than rates of link weights.

\subsubsection{Spike-Timing-Dependent Plasticity (STDP)}

It has been experimentally shown that the change in synaptic plasticity depends upon the relative spike timing between presynaptic and postsynaptic neurons. Spike timing dependence has become an important experimental tool for elucidating change in synaptic plasticity since its discovery [28]. Although the role of temporal order was suggested in the original statement of Hebb's rule, the time window requirement was not incorporated into the rule.

A variant of Hebbian learning, called spike-timing-dependent plasticity (STDP), has been proposed to explain such experimental phenomena. STDP is sensitive not only to the spatial but also to the temporal correlations between pre- and postsynaptic neurons. In STDP, a synaptic weight changes only if the time of the pre- and postsynaptic spikes occur sufficiently close in time [21]. We treat the time ranges of pre- and postsynaptic spikes as synaptic windows. In a synaptic window, the synapse will be potentiated if a presynaptic potential occurs before the postsynaptic spike, while the synapse will be depressed if a presynaptic potential occurs after the postsynaptic spike.

STDP has been extensively studied during the latest decade both experimentally and theoretically $[3,29,34]$. One line of computational research takes a given STDP rule and analyzes the evolution of synaptic efficacies $[30,35,36]$. But the functional consequences of these differences are largely unknown, as the exact time course of the STDP function varies between different types of neurons.

Various versions of STDP rules have been implemented in modeling studies to determine the effect on memory storage. Compared to the classical Hebb's rule, they have the advantage that 
there is strong competition between inputs, and the postsynaptic firing rate is independent of synaptic input rate. However, they are still unstable and finally result in a bimodal distribution of the synaptic weights [3]. Such a distribution is unlikely to occur in a biological neural network.

A mathematical model of STDP was proposed by Rossum, Bi and Turrigiano in 2000 [3]. The weight changes of the synapses are calculated according to the STDP rule in the model. We use $W$ to represent the weight of the synaptic connection, $W \rightarrow W+W_{p}$ for potentiation and $W \rightarrow W+W_{d}$ for depression, where

$$
\begin{gathered}
W_{p}=C_{p} \times e^{-\Delta t / \tau_{S T D P}}, \\
W_{d}=-C_{d} \times W \times e^{\Delta t / \tau_{S T D P}} .
\end{gathered}
$$

Here, $\Delta t$ is the time difference between the synaptic action and the postsynaptic spike, $\tau_{S T D P}$ is the time constant for depression and potentiation, $C_{d}$ is the average amount of the depression after one pairing connection, and $C_{p}$ is the average amount of the potentiation after one pairing connection. In the neuron system, the relative synaptic change is shown by the conductance change and it decreases exponentially within the time difference $\Delta t[3]$. Changing the synaptic weight of neurons can cause growth or decrease of activity in the neural network [31]. Balancing the extent of the inhibitory and excitatory links serves to maintain the network at an optimal level of activity $[37]$.

A stable distribution of the excitable link weights has been observed in Rossum's STDP model. In their calculations, each neuron receives input from 25 inhibitory and 100 excitatory synapses, and only the excitatory synapses change according to the spike timing. The inhibitory synapses have constant weight. Equations 2.1 and 2.2 are used for the potentiation and depression of the excitable links, respectively. 


\section{Reference}

[1] J. Horgan, "Can science explain consciousness?" Sci Am. 271, 88-94 (1994).

Online Version 23

[2] D. O. Hebb, The Organization of Behaviour: A Neuropsychological Theory (John Wiley \& Sons, New York, 1949).

Online Version 23, 30, 31

[3] M. C. W. van Rossum, G. Q. Bi, and G. G. Turrigiano, "Stable Hebbian learning from spiketiming-dependent plasticity," J. Neurosci. 20, 8812-8821 (2000).

Online Version 23, 31, 32, 35, 36

[4] M. E. J. Newman, "The structure and function of complex networks," SIAM Rev. 45, 167-256 (2003).

Online Version 24, 25, 26, 29

[5] M. E. J. Newman, "Models of the small world: A review," J. Stat. Phys. 101, 819-841 (2000). Online Version 24, 25

[6] D. Watts and S. Strogatz, "Collective dynamics of 'small-world' networks," Nature 393, 440442 (1998).

Online Version 24, 25

[7] S. Strogatz, "Exploring complex networks," Nature 410, 268-276 (2001).

Online Version 25, 26

[8] G. Palla, I. Derenyi, I. Farkas, and T. Vicsek, "Uncovering the overlapping community struc- 
ture of complex networks in Nature and society," Nature 435, 814-818 (2005).

Online Version 25

[9] J. M. Montoya and R. V. Sole, "Topological properties of food webs: From real data to community assembly models," Oikos 102, 614-622 (2003).

Online Version 26

[10] R. J. Williams and N. D. Martinez, "Simple rules yield complex food webs," Nature 404, 180-183 (2000).

Online Version 26

[11] A. Broder, R. Kumar, F. Maghoul, P. Raghavan, S. Rajagopalan, R. Stata, A. Tomkins, and J. Wiener, "Graph structure in the web," Computer Networks 33, 309-320 (2000).

Online Version 26

[12] I. R. Fiete, Learning and coding in biological neural networks, Ph.D. thesis, Harvard University (2003).

Online Version 26, 32

[13] M. Wilson, "Neuropsychology: Structure and function of a neuron," Technical report, http://issuu.com/neuropsychology/docs/lecture-3-pdf (2010).

Online Version 27

[14] K. W. Fischer, "A theory of cognitive development: The control and construction of hierarchies of skills," Psychol. Rev. 87, 477-531 (1980).

Online Version 26, 29

[15] N. Spruston, "Pyramidal neurons: dendritic structure and synaptic integration," Nat. Rev. 
Neurosci. 9, 206-221 (2008).

Online Version 27

[16] R. Rojas, Neural Networks: A Systematic Introduction (Springer, 1996).

Online Version 28, 32, 34

[17] E. M. Izhikevich, Dynamical Systems in Neuroscience: The Geometry of Excitability and Bursting (The MIT Press, 2006). 27, 32, 33

[18] B. Müller, J. Reinhardt, and M. T. Strickland, Neural Networks: An Introduction (Physics of Neural Networks) (Springer, 2002).

Online Version 29

[19] S. N. Williams, C. J. Locke, A. Braden, K. A. Caldwell, and G. A. Caldwell, "Epileptic-like convulsions associated with LIS-1 in the cytoskeletal control of neurotransmitter signaling in Caenorhabditis elegans," Hum. Mol. Genet. 13, 2043-2059 (2004).

Online Version 29, 30

[20] H. Berry and O. Temam, "Characterizing self-developing biological neural networks: A first step towards their application to computing systems," in "Lecture Notes in Computer Science," Vol. 3512, 306-317 (2005).

Online Version 29, 30

[21] W. Gerstner and W. M. Kistler, Spiking Neuron Models (Single Neurons, Populations, Plasticity), 10.3 Spike-Time Dependent Plasticity (Cambridge University Press, 2002).

Online Version 29, 35

[22] C. Koch and G. Laurent, "Complexity and the nervous system,," Science 284, 96-98 (1999). Online Version 29 
[23] O. Sporns, G. Tononi, and G. Edelman, "Theoretical neuroanatomy: Relating anatomical and functional connectivity in graphs and cortical connection matrices," Cereb. Cortex 10, $127-141(2000)$.

Online Version 29

[24] O. Sporns, "Network analysis, complexity, and brain function," Complexity 8, 56-60 (2002). Online Version 29

[25] J. G. White, E. Southgate, J. N. Thomson, and S. Brenner, "The structure of the nervous system of the nematode Caenorhabditis elegans," Philosophical Transactions of the Royal Society B: Biological Sciences 314, 1-340 (1986).

Online Version 30

[26] K. Oshio, Y. Iwasaki, S. Morita, Y. Osana, S. Gomi, E. Akiyama, K. Omata, K. Oka, and K. Kawamura, "Database of synaptic connectivity of $C$. elegans for computation," Technical Report of CCeP, Keio Future No. 3, Keio University, Japan (2003).

Online Version 30

[27] L. N. Cooper, "Memories and memory: A physicist's approach to the brain," Int. J. Mod. Phys. A 15, 4069-4082 (2000).

Online Version 31

[28] T. V. Bliss and T. Lomo, "Long-lasting potentiation of synaptic transmission in the dentate area of the anaesthetized rabbit following stimulation of the perforant path," J. Physiol. (Lond.) 232, 331-356 (1973).

Online Version 31, 35

[29] Y. Dan and M. M. Poo, "Spike timing-dependent plasticity of neural circuits," Neuron 44, 
23-30 (2004).

Online Version 31, 35

[30] L. J. Bindman, K. P. Murphy, and S. Pockett, "Postsynaptic control of the induction of long-term changes in efficacy of transmission at neocortical synapses in slices of rat brain," J. Neurophysiol. 60, 1053-1065 (1988).

Online Version 31, 35

[31] T. P. Vogels, K. Rajan, and L. Abbott, "Neural network dynamics," Annu. Rev. Neurosci. 28, 357-376 (2005).

Online Version 32, 36

[32] W. McCulloch and W. Pitts, "A logical calculus of the ideas immanent in nervous activity," Bull. Math. Biophys. 5, 115-133 (1943).

Online Version 33

[33] L. Qian, E. Winfree, and J. Bruck, "Neural network computation with DNA strand displacement cascades," Nature 475, 368-372 (2011).

Online Version 33

[34] S. Song, K. D. Miller, and L. F. Abbott, "Competitive Hebbian learning through spike-timingdependent synaptic plasticity," Nat. Neurosci. 3, 919-926 (2000).

Online Version 35

[35] H. Markram, J. Lbke, M. Frotscher, and B. Sakmann, "Regulation of synaptic efficacy by coincidence of postsynaptic APs and EPSPs," Science 275, 213-215 (1997).

Online Version 35 
[36] L. I. Zhang, H. W. Tao, C. E. Holt, W. A. Harris, and M. M. Poo, "A critical window for cooperation and competition among developing retinotectal synapses," Nature 395, 37-44 (1998).

Online Version 35

[37] R. Segev, M. Benveniste, Y. Shapira, and E. Ben-Jacob, "Formation of electrically active clusterized neural networks," Phys. Rev. Lett. 90, 168101-1-4 (2003).

Online Version 36 


\section{Chapter 3}

\section{A Trainable Chemical Neural Network: Experiments and Simulations}

Fundamentally, neurons are autonomous relaxation oscillators, and connected groups of neurons in separated brain regions act as autonomous phase oscillators. Systems of differential equations and cellular automata have been proposed to simulate the behavior of neurons in brain. Very popular are Fitzhugh-Nagumo dynamics and Hodgkin-Huxley [1, 2] systems of differential equations, originally suggested to analyze a quantitative description of the membrane current in neurons. Excitable media was first investigated in a biological context by Wiener and Rosenblueth [3], who modeled waves rotating around obstacles in cellular automata models of excitable cardiac muscle. Early computer simulations were performed by Farley [4], who laid out a virtual neural network on a rectangular grid.

In these models, a number of cells in the excited state induce a resting cell to the excited state. A weighting function of a cell's relative contribution to the excitation decreases as a function of distance. Gerhardt and colleagues [5, 6] used an excitation threshold that was a decreasing function of time. Marcus and Hess [7] and Kurrer and Schulten [8] randomized the grid in circular 
or square neighborhoods. To diminish the difference between differential equation and cellular automata approaches, continuous differential equation models began to be used in each element of the cellular automaton grid [9-11].

However, experimental observations in excitable systems on the behavior of biological neural networks were rarely reported. The excitable features of BZ systems suggest that a network of BZ nodes can model a neuronal network. We have successfully developed a network in a BZ discrete reaction system, where excitable particles are the nodes. The experimental system can mimic synapse dynamics, and evolve (learn) by a STDP rule. In this chapter, the framework of the network in the BZ reaction is defined, and the dynamics of the overall network is studied. Although the behavior of an individual excitable particle in the BZ system is simple, we are interested at how a population of particles in a network can behave together through different types of links.

\subsection{Experimental Setup}

A schematic representation of the experimental setup is shown in Fig. 3.1. Basically, there are three parts: the chemical system where the BZ reaction takes place; the instrumentation setup to control the illumination and record the dynamics of the particles in the photosensitive BZ reaction, and the network setup.

\subsubsection{Preparation of the Chemical System}

The BZ reaction takes place in a glass vessel, containing the catalyst-free BZ solution: $0.166 \mathrm{M}$ $\mathrm{NaBrO}_{3}, 0.054 \mathrm{M}$ malonic acid, $0.162 \mathrm{M}$ bromomalonic acid, and $0.366 \mathrm{M} \mathrm{H}_{2} \mathrm{SO}_{4}$. The particles

are loaded with the catalyst $\left[\mathrm{Ru}(\mathrm{bpy})_{3}^{2+}\right]$. The catalyst-loaded particles are prepared according to the recipe in Ref. [12]. Cation exchange particles, Bio-Rad analytical grade 50W-X (50 - 100 


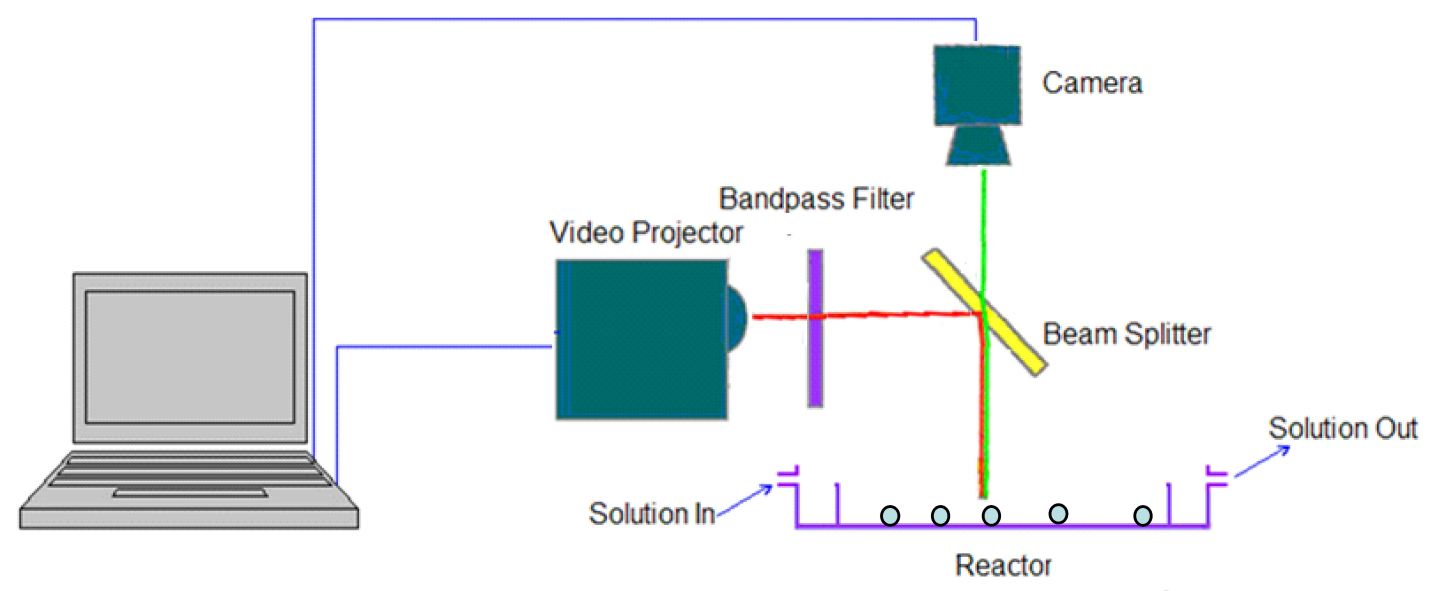

Figure 3.1. Experimental setup for the BZ network.

mesh), were purchased from Fisher Scientific. A $3 \mathrm{~g}$ quantity of particles are stirred in a $4 \mathrm{ml}$ solution of $25 \mathrm{mM}\left[\mathrm{Ru}(\mathrm{bpy})_{3}^{2+}\right]$. After $7-8$ hours, particles are removed and left to dry on filter paper. The catalyst concentration was calculated to be $1.7 \times 10^{-5} \mathrm{~mol} \mathrm{~g}^{-1}$.

In the experiment, particles loaded with the catalyst $\left[\mathrm{Ru}(\mathrm{bpy})_{3}^{2+}\right]$ are randomly placed in the bottom of the vessel with sufficient distance between them to avoid any interactions. The stock catalyst-free BZ solution, maintained in an ice bath, was continuously pumped into the reactor vessel at a flow rate of $0.8 \mathrm{mlmin}^{-1}$ using a peristaltic pump (Ismatec). The reaction mixture was also pumped out at the same rate, so the solution depth in the reactor vessel was maintained constant during an experiment. Two manifolds are located at the inlet and the outlet to avoid motion of particles during the experiments. The temperature of the reaction solution is controlled at $35^{\circ} \mathrm{C}$ by a water bath. 


\subsubsection{The Instrumentation Setup}

As shown in Fig. 3.1, an illumination profile in the experiment is produced by a modified video projector, which is controlled by a computer coupled to a CCD video camera fitted with a macro lens.

The light from the projector is scattered from a white membrane filter (Pall Gelman Laboratory, Super-450, $0.45 \mathrm{~m}$ ) positioned underneath the reactor. This also serves as the background for observing the chemical oscillators. Light at $440-460 \mathrm{~nm}$, corresponding to the absorption band of ruthenium(II), is selected by a bandpass filter. Particles can be illuminated independently by the projector according to a computed illumination intensity. The beam splitter reflects the light onto the particles, while allowing observation with the camera. Images of the chemical state of the beads are collected by the camera and sent to the computer for calculation of the real-time feedback of the illumination.

Concentrations are selected so that under bright-light conditions of $\phi_{\text {high }}=1.38 \mathrm{~mW} \mathrm{~cm}^{-2}$,

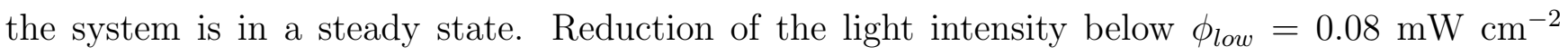
leads to an excitable excursion. A CCD camera records the position and intensity of the particles [13]. With the coupling turned off, images of the reaction domain are obtained using a projected background light intensity onto each particle of $\phi_{b g d}=0.76 \mathrm{~mW} \mathrm{~cm}^{-2}$. Images are captured every 2.0 seconds. Any oscillations are accompanied by changes in the oxidized state of the catalyst $\left[\mathrm{Ru}(\mathrm{bpy})_{3}^{2+}\right]$ and are monitored by the changes in the measured light intensity of a particle.

\subsubsection{The Network Setup}

From Chapter 2, we know that the biological neural network has three primary components or functions: independent neurons as the nodes of the network, synapses as connections between 


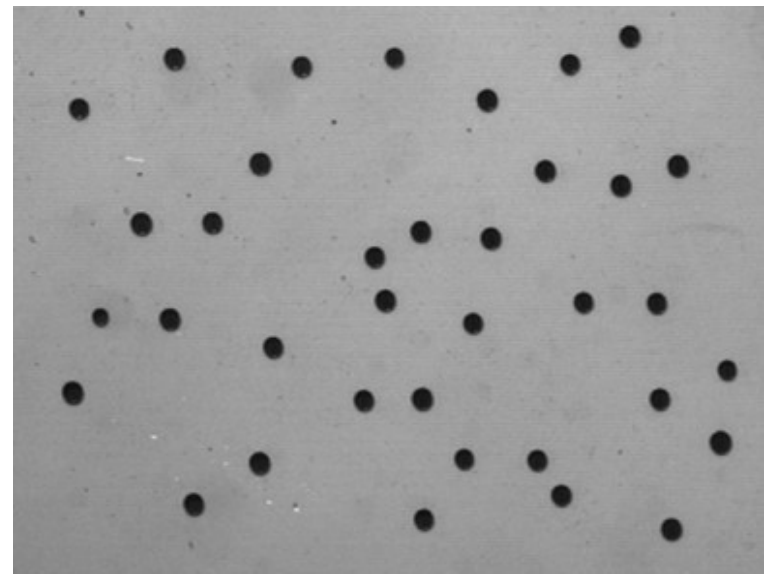

Figure 3.2. Particles reacting in BZ solutions as the nodes in the chemical network. The ionexchange resin particles, with size of $50-100$ mesh, are loaded with the catalyst $\left[\mathrm{Ru}(\mathrm{bpy})_{3}^{2+}\right]$.

neurons to transmit the signals, and spikes generated from neurons as the signals [14]. Thus, in order to create a chemical model system, we need these components in our network: nodes, weighted links behavioring like synapse, and some form of firing events as spikes.

Here, as shown in Fig. 3.2, the nodes in our network are defined as the independent ion-exchange particles $\left(50-100\right.$ mesh), loaded with $\left[\mathrm{Ru}(\mathrm{bpy})_{3}^{2+}\right]$ catalyst and reacting in a catalyst-free BZ solution. The particles are positioned so the diffusion of active species from one particle to the next is negligible. Each reacting particle represents a neuron in a biological neural network. The particles can be in the excitable or oscillatory states depending on their illumination. The firing event is defined as an oscillation of a particle in the oscillatory state.

In a biological neural network, each neuron is connected with more than 1000 other neurons. To decrease the complexity of the analysis in our network, we set the average number of links from a node into $\sim 20$. There are also two types of links in our network. One is the excitatory link, with a weight of 0.04 , and the other one is the inhibitory link, a the weight of -0.08 . The ratio of the number of excitatory links to the number of inhibitory links is $4: 1$, which has been used in other 
neural network models $[15,16]$.

In the experiment, the locations of the particles are first determined through analyzing the image from the camera by the computer. As shown in Fig. 3.3, typical experiments involve 49 particles, which are mapped onto virtual network, with 980 links between the particles. The excitatory links are $80.0 \%$, with weight of 0.04 , and the inhibitory links are $20.0 \%$, with weight of -0.08. The probability of a connection between node $i$ and $j$, given by $P\left(r_{i, j}\right)$, decreases exponentially with distance from the source node:

$$
P\left(r_{i, j}\right)=\frac{1}{\sigma \sqrt{2 \pi}} e^{\frac{-r_{i, j}^{2}}{2 \sigma^{2}}}
$$

Different values of the standard deviation were used for the excitatory and inhibitory links: $\sigma_{e}=3$, $\sigma_{i}=5$. The information of the nodes and the links is stored in the computer.

In the logical array set up from the network, the distance between two immediate horizontal or vertical neighbors is set as 1 . The links are then arranged according to the probability of the distances between any two nodes according to a normal distribution. The detailed process of arranging a link is shown in Fig. 3.3. First, a node is randomly selected as the source of the link. Second, a value, determined by the distance of the link, is selected according to the normal distribution function. Third, the distance is projected in both the horizontal and vertical directions with a randomly selected angle. The destination node of the link is chosen as the one that lies closest to this vector. The distance value is generated by the normal distribution function, so smaller values have higher probability to be selected [17].

In our network, a computer stores the link weights of all nodes and counts the triggered firing events from the nodes. Thus, the STDP algorithm can be implemented, with the processes of potentiation and depression. After firing events occur, the computer updates the matrix of the link weights according to the relationship between the pre- and post-node by the STDP rule. 


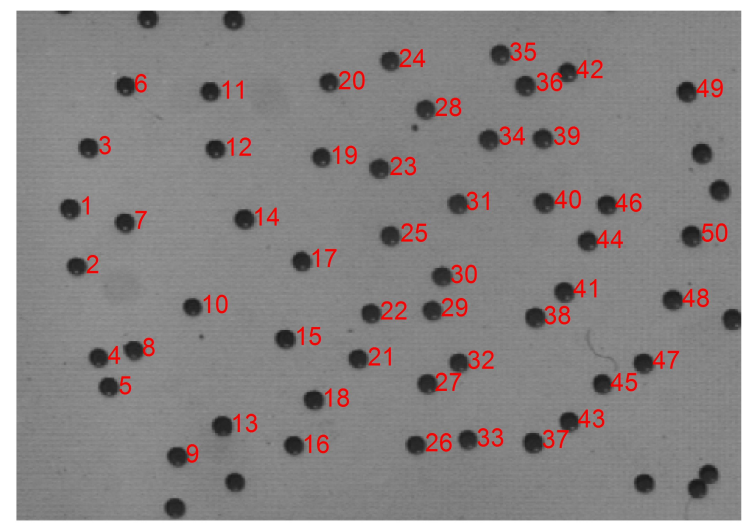

(a)

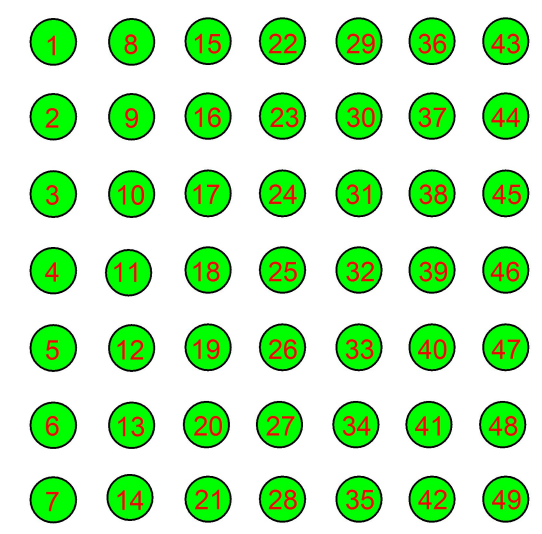

(b)

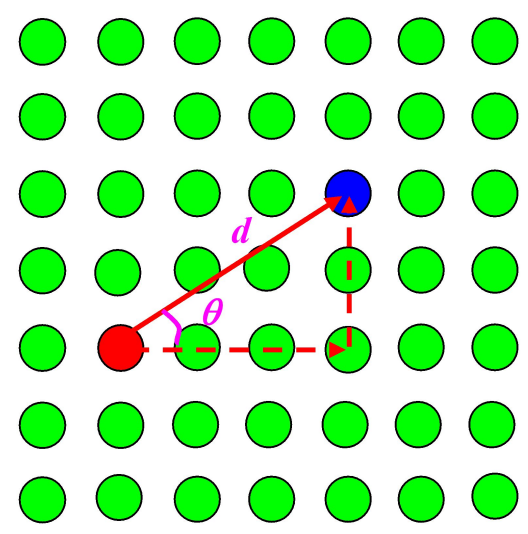

(c)

Figure 3.3. Arrangement of links in the chemical system. (a) The numbered particles in the experiment. (b) A virtual array composed of the numbered particles as nodes in a network. (c) The process of assigning a link in the virtual array. Here, the source node is randomly selected and labeled as red, and the destination node is labeled as blue. The value of $d$ is generated from a normal distribution probability function with a random angle $\theta$. 


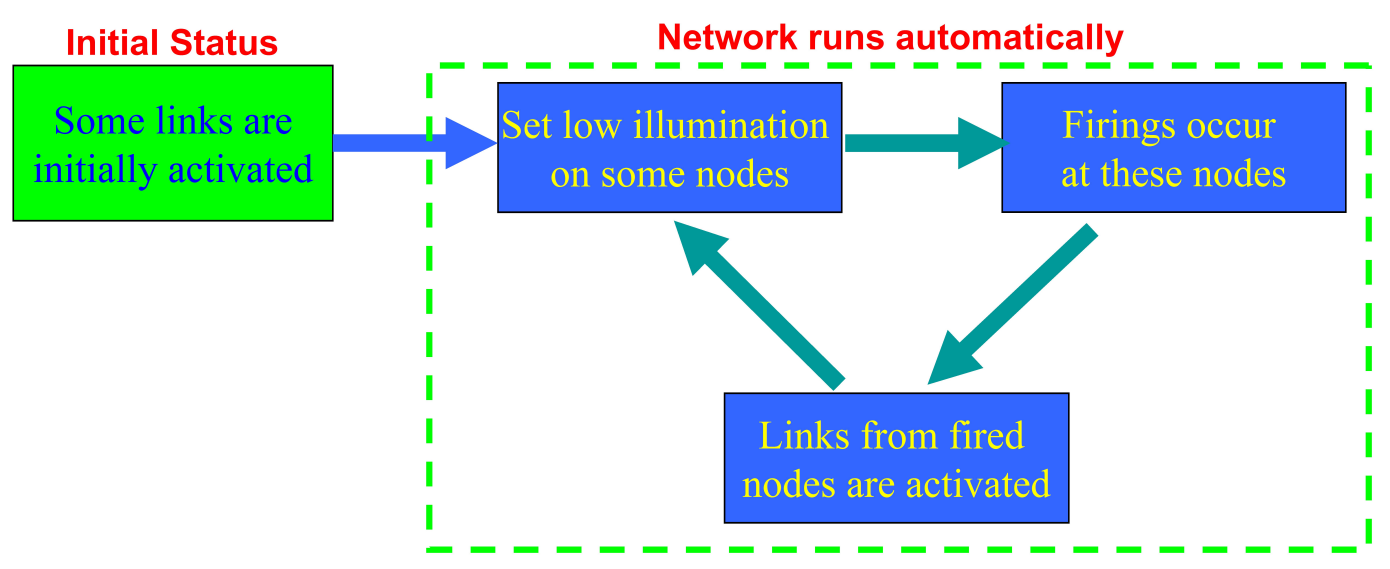

Figure 3.4. Diagram of training process.

\subsection{Experimental Results}

In principal, an artificial neuronal network can be trainable, where the network evolves based on specific learning rules and finally arrives at a certain stable state. The trainable process of our network is illustrated in Fig. 3.4. Initially, some links are activated (i.e., initial activity is induced). The network then continues with the STDP rule. The updated link weight matrix is recorded in the computer in each step. The initial activity sets the links from $10-20 \%$ of randomly selected nodes to be active, and the life time for activated links is set. These settings are garanteed to initially cause some particles to fire. The final status of the network is one of two states: activity exists or has collapsed. Zero activation corresponding to a global excitable steady state, with high illumination on all oscillators. The nature of the sustained activity state is what we investigate in this network.

In a typical artificial neuronal network, the node integrates input and then performs a firing event. The weights of the links on each node will be integrated at any time. As soon as the sum of links of a node reaches a threshold value, a firing event occurs. However, no more firing events can be activated during the refractory period. 


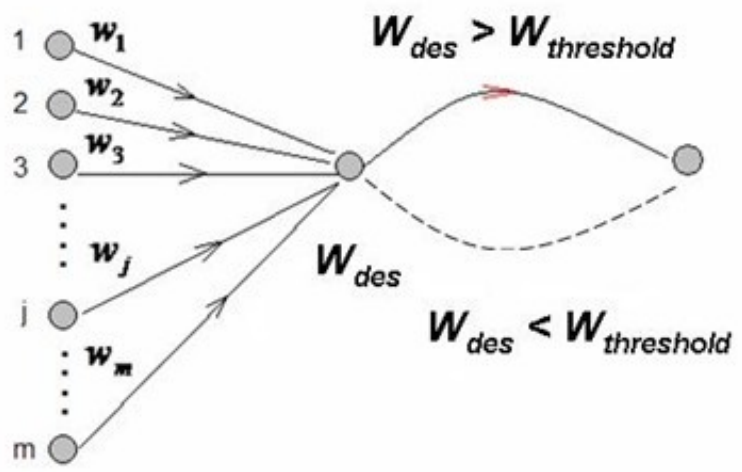

(a)

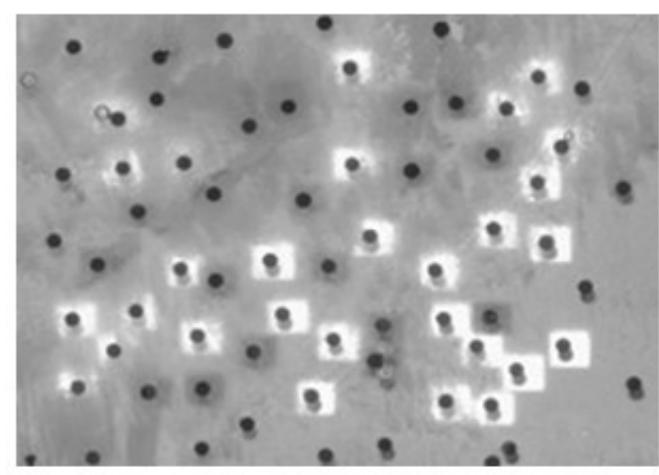

(b)

Figure 3.5. Effect of links on the oscillator illumination. (a) Function of Links: integrate illumination. If the sum of active links on a destination node, $W_{d e s}$, is greater than the threshold, $W_{\text {threshold }}$, the illumination of the node is set at the high light intensity; otherwise, its illumination is set as the low value. (b) Illuminations of the nodes. The light dots are the particles illuminated with high light intensity and the dark dots are the particles illuminated with low light intensity.

In our network, links are represented in two states, active or inactive. At any time, only active links affect the system behavior, while inactive links can be activated by firing events. As soon as a node triggers a firing event, all of the links originating from the node are activated. The newly activated links will become inactive again after a preset life time.

The firing events, the oscillations of the perturbed particles reacting with the BZ solution, can not be triggered by simply counting the sum of the active link weights at any time. Thus, the function of the links in our network is used to determine the illumination intensity on each particle, as shown in Fig. 3.5. As expected, a firing event can occur only when a node have been exposed to low illumination intensity for sufficient time. The occurrences of firing events in four different conditions are described in the photosensitive Oregonator model in Fig. 3.6. Figure 3.6a shows that there are no firing events when a node is always illuminated at high intensity (illumination 


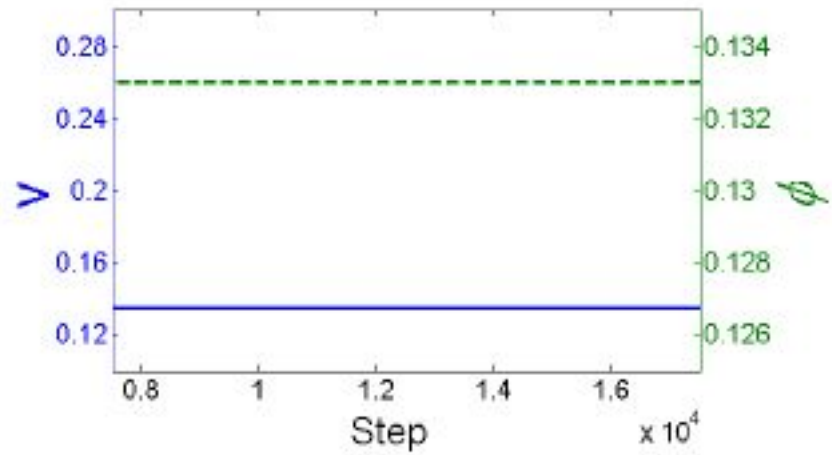

(a)

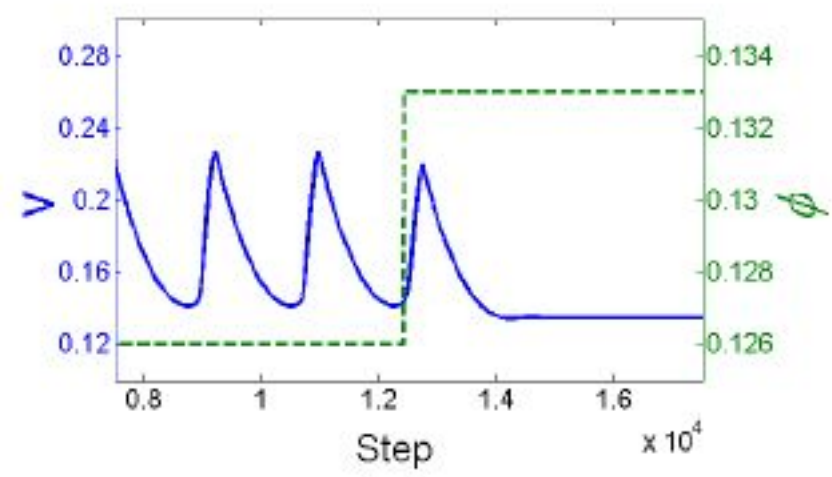

(c)

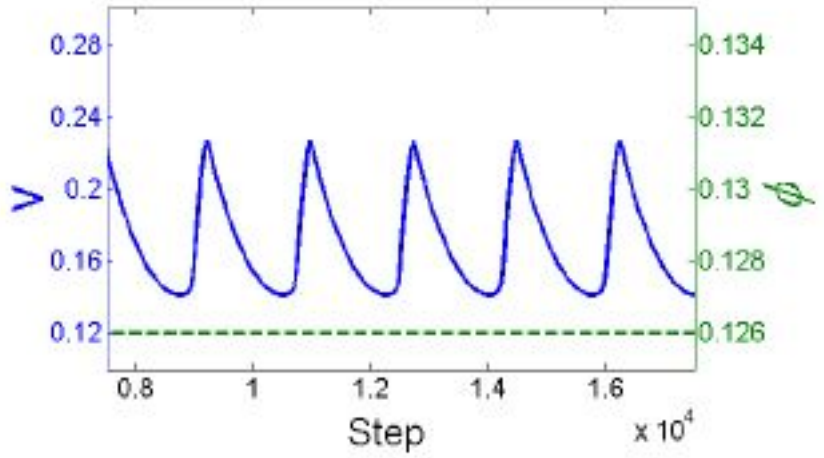

(b)

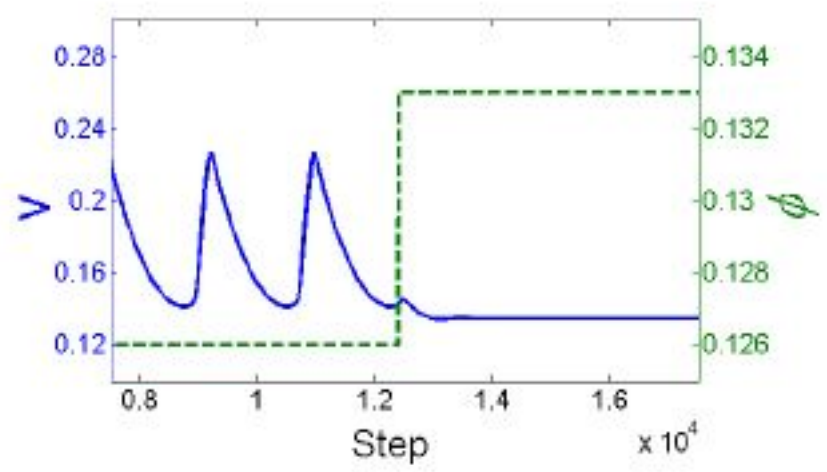

(d)

Figure 3.6. Firing events in the photosensitive Oregonator model. (a) High illumination $=$ 0.0133; (b) low illumination $=0.0126$; (c) illumination is switched from low to high at step $=$ 12400; (d) illumination is switched from low to high at step $=12380$. Simulation conditions: $d x$ $=0.15, d t=0.001, q=0.05, \epsilon=0.005, f=0.9, D_{u}=1.0$, and $D_{v}=0$. 


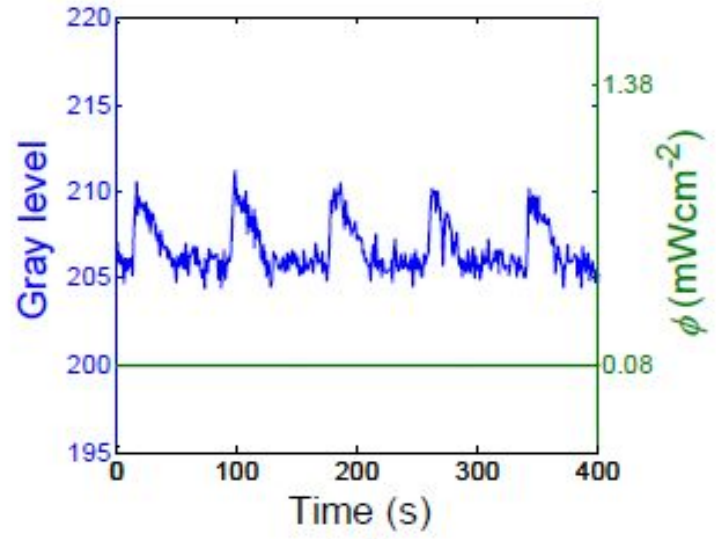

(a)

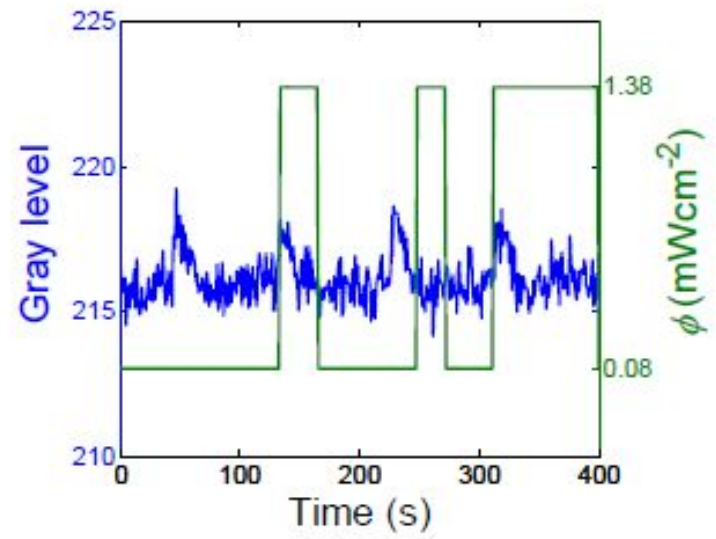

(c)

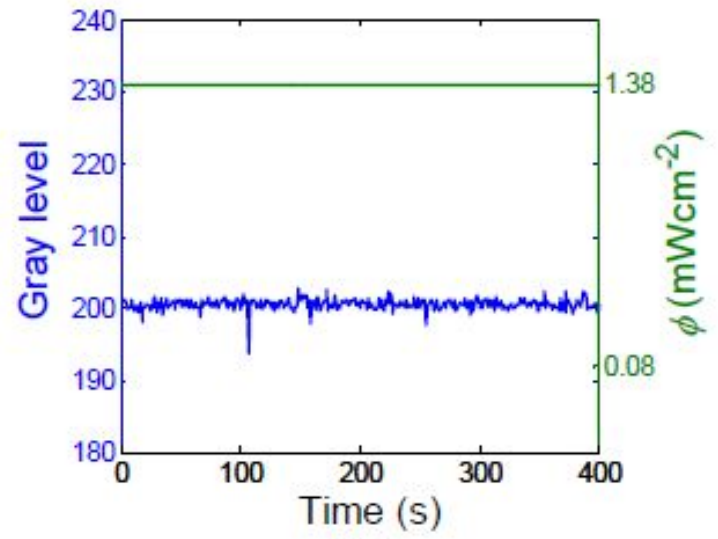

(b)

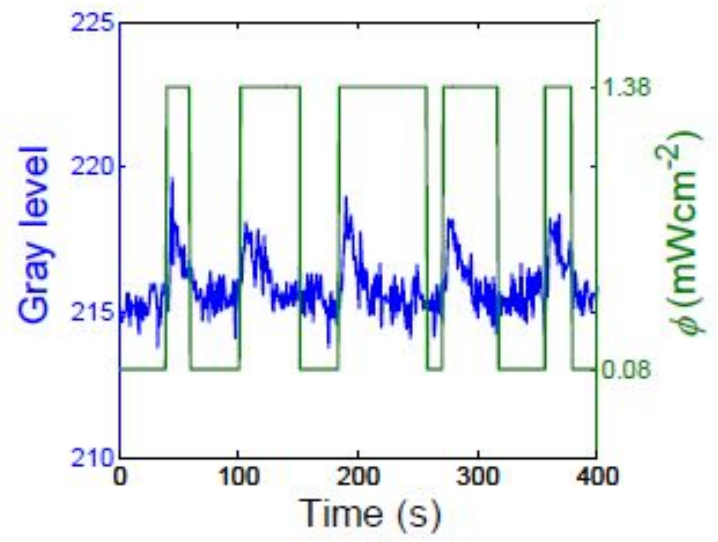

(d)

Figure 3.7. The behaviors of nodes with different illumination patterns.

$=0.0133)$. An array of firing events is monitored as shown in Fig. 3.6b, during a brief period of background intensity illumination.

Figure 3.7 shows the behavior of nodes subject to different illumination patterns. Figure 3.7a shows the behavior of a node with low illumination intensity. A series of continuous oscillations are observed on this node, indicating that the node is in the oscillatory state. Figure 3.7b shows the behavior of a node with high illumination intensity. The collected signals indicate that the node is in the steady state without oscillations. During an actual experiment, the active links are updated based on the occurrence of new firing events; the illumination array of a node is 
switched between the low value and the high value. Some illumination sequences will favor the occurrence of firing oscillations. Figure 3.7c and 3.7d show two such illumination arrays for nodes, where some firing events have been observed. Most of firing events require some low illumination intensity. However, the illumination arrays with low light intensity are rarely observed and the illumination arrays are often separated by different segments of low intensity and high intensity. The switch between low illumination and high illumination is favorable for producing oscillations. Specifically, a perturbation that is a short-time low illumination intensity, as in Fig. 3.7d, also triggers a firing event. In 2001, Wang found that an oscillation can be triggered in the BZ system by pulse-light perturbation [18]. The situation in our work is more complicated because different segments of low illumination intensity and high illumination intensity are combined. The combined illumination sequences can trigger firing events but the time intervals between the oscillations may be different. For example, the interval between the first oscillation and the second oscillation is shorter compared with other intervals in Fig. 3.7d.

The period of oscillation in the STDP system is shown in Fig. 3.8 compared with the period of oscillation in the system without STDP. Figure 3.8a shows the distribution of the period of oscillation for all nodes with constant low illumination. Figure 3.8b shows the distribution of the period of oscillation in the STDP network, which is much broader. The reason may lie in the occurrence of firing events in the two system due to the induction of low and high illumination intensity.

In Figure 3.9, we characterize the behavior of the oscillators during sustained firing in the experimental system. Figure 3.9a shows typical firing patterns of the individual oscillators. The firing events are irregular with intermittent quiescent periods and periods of regular firings. This results in periodic oscillations in the global activity of the system. Figure 3.9b illustrates this global behavior with the total system activity plotted as a function of time. Fourier analysis reveals the 


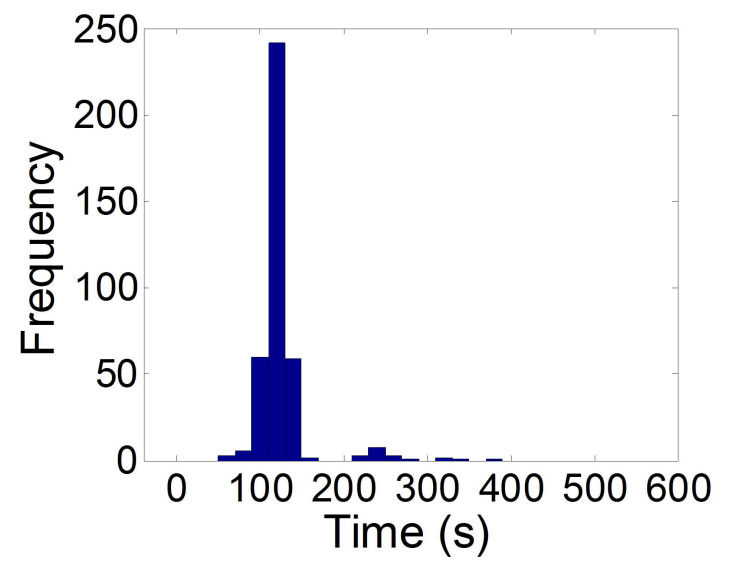

(a)

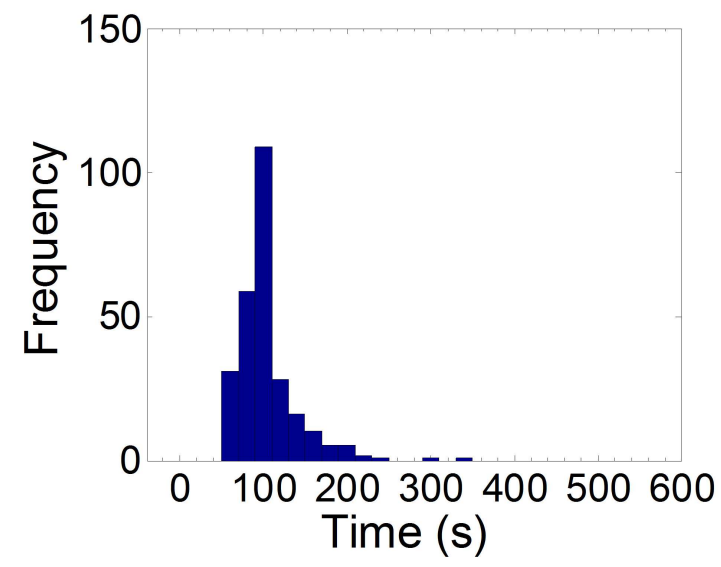

(b)

Figure 3.8. The period of oscillation in a network illuminated with $\phi_{\text {low }}=0.08 \mathrm{~mW} \mathrm{~cm}^{-2}$ (a) and in an STDP network (b).

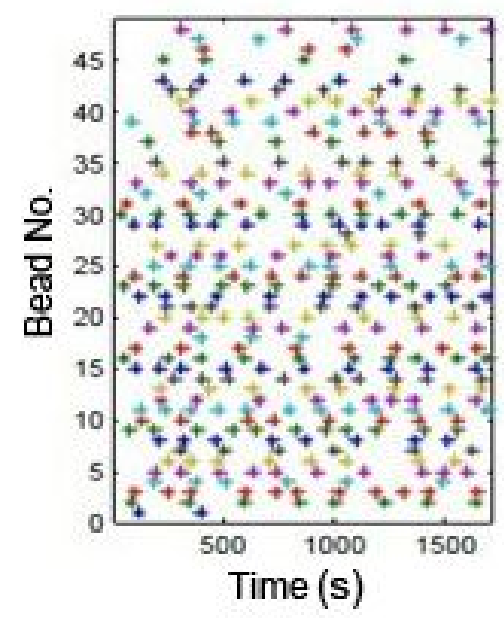

(a)

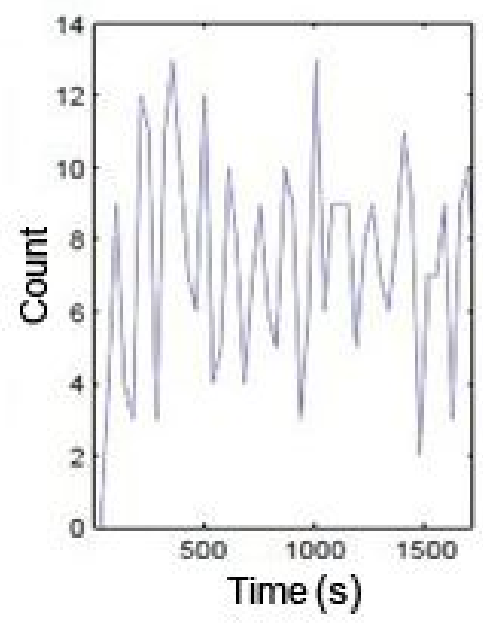

(b)

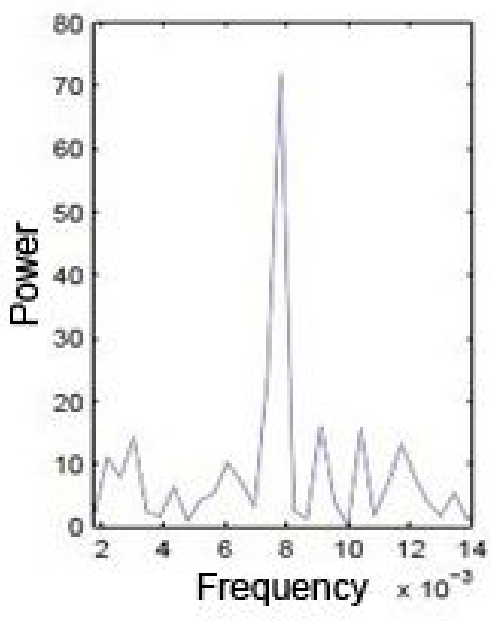

(c)

Figure 3.9. Experimental system firing pattern. (a) The firing patterns of the 49 oscillators.

Each cross indicates the time at which an individual oscillator fires. (b) Time series showing the number of oscillators that fire in the given interval of time. The count is constructed by splitting the entire time range into bin intervals of 20 s and then determining how many oscillators fire in each interval. (c) Power spectrum of the Fourier transform of the time series. 


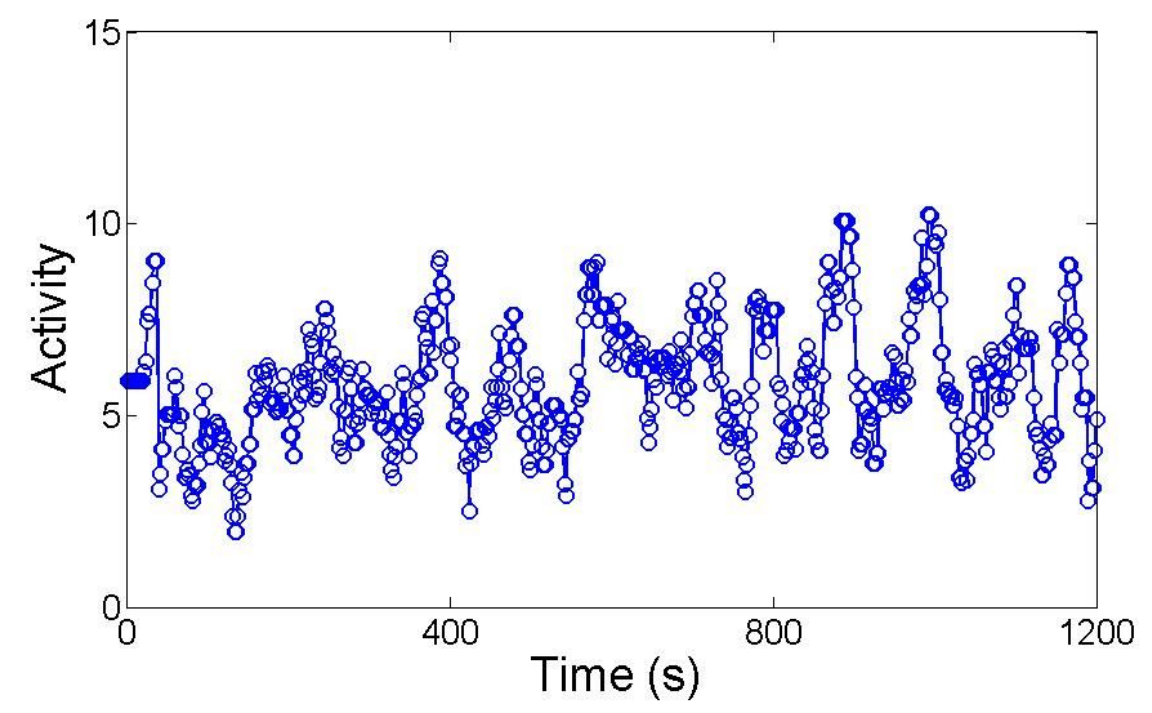

Figure 3.10. The activity of the STDP system as a function of time.

presence of a dominant frequency in the time series shown in Fig. 3.9c. Each of the dominant frequencies is associated with the value of $t_{\text {link }}=80 \mathrm{~s}$. The reciprocal of the dominant frequency, determined from the power spectrum, is $126 \mathrm{~s}$.

The activity of the STDP network, defined as the sum of all activated links, is also analyzed. Figure 3.10 shows the activity of the STDP system as a function of time. At the beginning of an experiment, activity results from the activation of $20.0 \%$ randomly selected nodes used to initiate the STDP system. A decreasing of the activity occurs after a short time from the initiation. This is because the life time of most links has expired and the links become non-active. A small peak of activity is then observed, indicating more firing events have occurred to activate more links. The total activity of the network roughly oscillates, indicating an approximately global periodcity.

Only the weights of excitatory links are adjusted by the STDP rule in this study. We are interested in the distribution of the excitatory links. In our experiments of training the STDP network, various of distributions of link weights can be obtained by adjusting the experimental conditions and STDP parameters. 


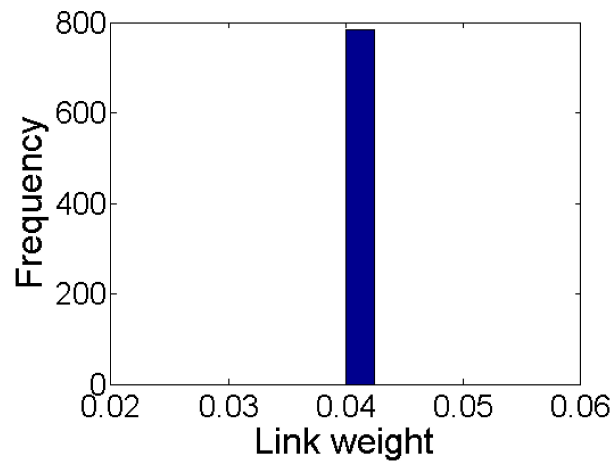

(a)

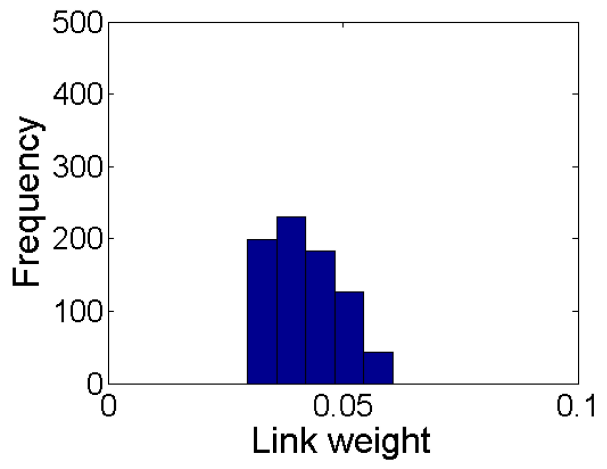

(c)

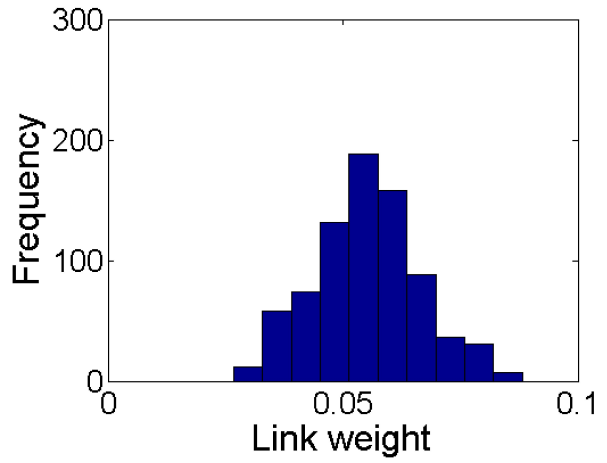

(e)

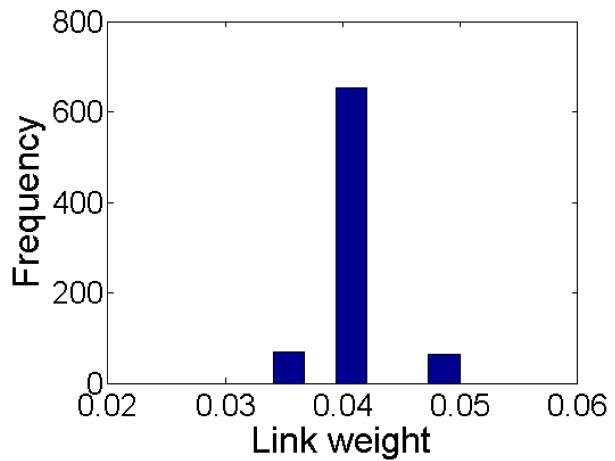

(b)

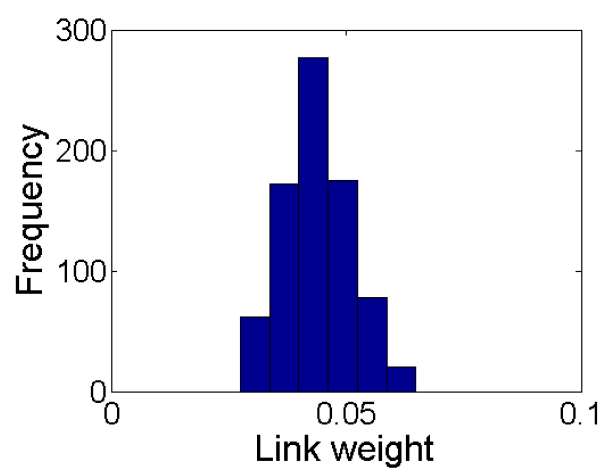

(d)

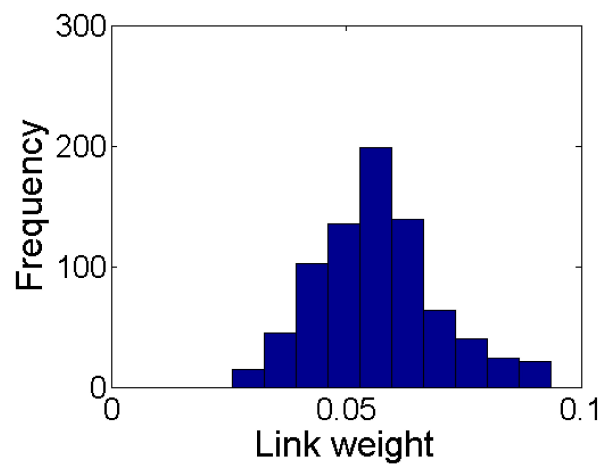

(f)

Figure 3.11. The distribution of weights of the excitatory links at different experimental times. 
A stable normal distribution of the weights of the excitatory links is acquired by adjusting the experimental parameters. Figure 3.11 shows the distributions of the weights of the excitatory links in different times. As shown, the distribution eventually reaches a stable normal distribution from the initial value of 0.04 .

\subsection{Simulations}

Simulations of the behavior are carried out using a two-variable Oregonator model for the photosensitive BZ reaction to represent the chemistry on each particle:

$$
\begin{aligned}
& \frac{\partial u}{\partial t}=D_{u} \nabla^{2} u+\frac{1}{\epsilon}\left\{u-u^{2}-[\phi+f v] \frac{u-q}{u+q}\right\}, \\
& \frac{\partial v}{\partial t}=D_{v} \nabla^{2} v+u-v
\end{aligned}
$$

where $u$ and $v$ are the dimensionless concentrations of $\mathrm{HBrO}_{2}$ and $\mathrm{Ru}\left(\mathrm{bpy}_{3}\right)^{3+}$ on a particle i. The rate of bromide production is $\phi$ due to the irradiation [19].

The Euler method is used for the numerical investigation, with $d t=0.001$. Typical simulations involved virtual grids of particles up to $30 \times 30$ in size. The network of links is established using the same distance dependent algorithm as for the experimental system. Model parameters are chosen so that each oscillator is in a steady state for $\phi_{b g d}=0.15$. Reduction of the light intensity below $\phi=0.02$ leads to an excitation excursion. Activity is initiated in the network by reducing $\phi$ to zero on the central particle for 250 time steps.

At each time step, we determine if an oscillator has recently fired by examining the value of the variable $v$. If an oscillator has fired, then all links originating at that particle are turned on for a set period of time $t_{\text {link }}$. The illumination to each particle is updated at each time step based on the sum of currently active links. Two illumination algorithms have been investigated: a discrete 


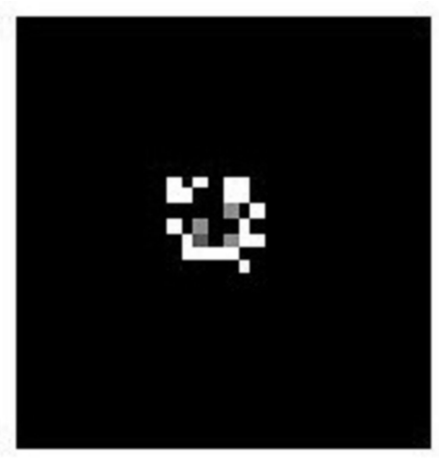

(a)

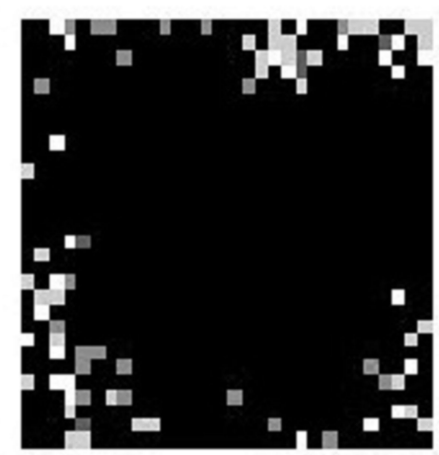

(c)

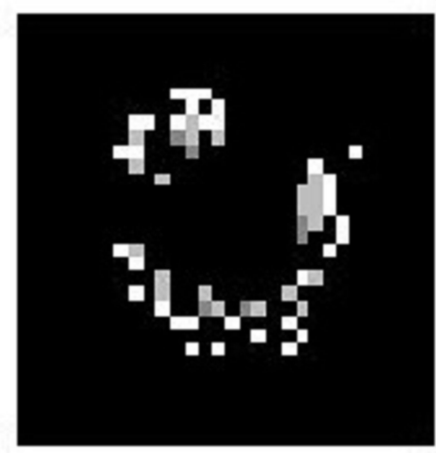

(b)

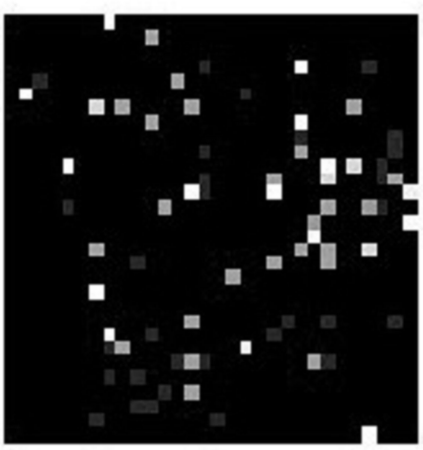

(d)

Figure 3.12. A patchy wave of activity propagating in the BZ network with STDP. After an initial perturbation at the center (a), activity spreads outward through the network (b) and finally reaches the edge of the network (c). Oppositely directed links initiate activity on particles that did not fire in the initial pass. These particles then reinitiate other particles, leading to a complex aperiodic activation pattern. System parameters: $\epsilon=0.01, f=1.4, q=0.002$, particles $=30 \times 30$, links $=22500, t_{\text {link }}=2, \sigma_{e}=2, \sigma_{i}=4, l_{\text {excitatory }}=0.04$ (excitatory), $l_{\text {inhibitory }}=-0.08$ (inhibitory), $\phi_{b g d}=0.15$, period of oscillation $=4$. 


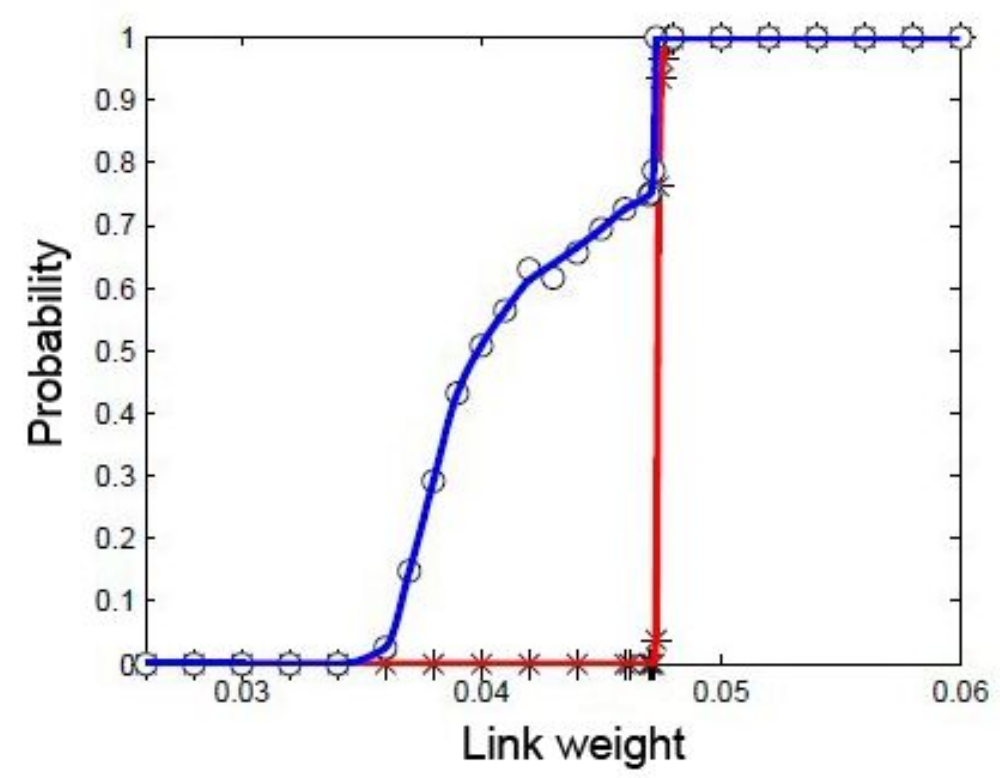

Figure 3.13. Blue crosses indicate constant link weight. Red circles indicate that the STDP algorithm is applied. All parameters as in Fig. 3.12. The probabilities are based on 500 simulations, each with a different link distribution for each link weight. The activity is determined to be sustained if it survives to $t=1000$.

state algorithm, as in the experiment system, and a continuous illumination algorithm given by

$$
\phi=\phi_{b g d}+\sum_{\text {active links }} l,
$$

with $\phi$ prohibited from becoming negative. Both techniques produce qualitatively similar results. Here we illustrate the behavior using the update algorithm given above.

System parameters in the simulation are initially tuned to obtain sustained behavior in the network following a perturbation at the center. In Fig. 3.12, a patchy wave of activity propagates from the center of the medium, reflects from the boundaries and eventually fills the network with incoherent spatiotemporal structures, Fig. 3.12d. The possibility of the initial perturbation propagating and then persisting is a complex function of a number of parameters associated with 


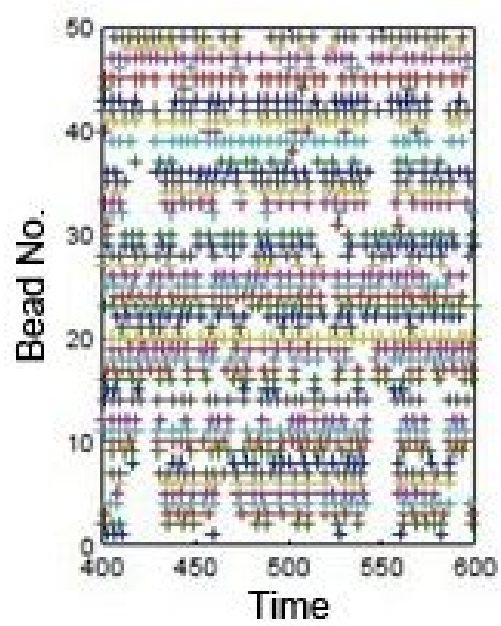

(a)

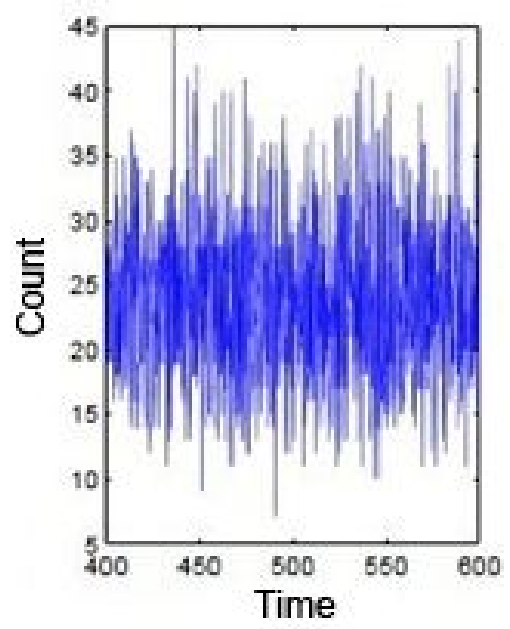

(b)

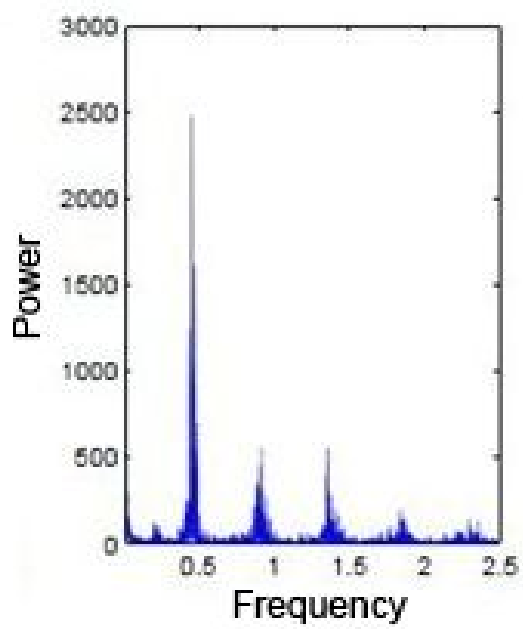

(c)

Figure 3.14. Numerical system firing pattern. (a) The firing patterns of 50 of the 900 oscillators.

(b) Time series showing the number of oscillators that fire in a given interval of time (bin interval $=0.2$. (c) Power spectrum of the Fourier transform of the time series.

the network. These include the intrinsic chemical timescale, the number of links, the lifetime of a link, the weight of a link and the magnitudes of $\sigma_{e}$ and $\sigma_{i}$. At low values of link weight and link number, the initial perturbation to the system dies out quickly with the system returning to its quiescent state. For large values of $\sigma_{e}$ and number of links, the system becomes effectively globally coupled, leading to a single collective oscillation. At intermediate values, activity spreads from the center of the system, as shown in Fig. 3.12. The presence of the inhibitory links leads to breaks in the initial spread of activity. Under appropriate choice of other system parameters, such as $t_{\text {link }}$, reinjection into the region where activity has recently passed can occur. The possibility of the activity then becoming sustained is also sensitive to the choices of parameters. For example, a sharp transition to sustained behavior with increasing link weight is shown in Fig. 3.13. On the left of this figure, the quiescent state is globally stable, whereas on the right, there is bistability between the quiescent state and the sustained state. 
As shown in Fig. 3.14, the behavior of the oscillators during sustained behavior in the computational system is similar to that in the experiments. Here, the firing is also irregular with intermittent quiescent periods and periods of regular firings. The noisy oscillation in the global behavior is approximately twice the frequency of the component individual oscillators.

The longer term behavior associated with this algorithm can be explored further using the numerical system. Starting with a system in which $l=0.04$ for all excitatory links, the application of the STDP algorithm leads to a gradual increase in the mean value of the link weights. The resulting stable skewed distribution is shown in Fig. 3.15a. For the same set of parameters, but with a higher initial excitatory link weight, $l=0.12$, the mean link weight now slowly decreases to eventually produce a stable skewed distribution, Fig. 3.15b. These two final distributions are overlaid in Fig. 3.15b, and they are seen to have approximately the same mean value and overall shape. This remarkable agreement between these distributions indicates that the distribution behaves as an attractor of the system with the STDP algorithm.

A necessary condition for the STDP algorithm to produce a stable distribution of link weights is that the activity on the network must be sustained. In Fig. 3.13, the probability of sustained behavior under constant excitatory link weight conditions was shown as a function of this link weight. A sharp transition in the probability of sustained behavior occurs on increasing link weight. The impact of allowing link weight evolution, using the STDP algorithm, on these results is shown in Fig. 3.13. In this case, the link weight on the $x$-axis now corresponds to the initial excitatory link weight. It can be clearly seen from this graph that the STDP algorithm softens the steepness of transition and expands the region in which sustained behavior is observed. A significant proportion of the simulations with an initial link weights below 0.047 (the critical transition link weight for the constant link weight simulations) have sustained behavior. The expansion of the region in which sustained behavior is seen can be understood with the help of Fig. 3.15. We consider a situation 


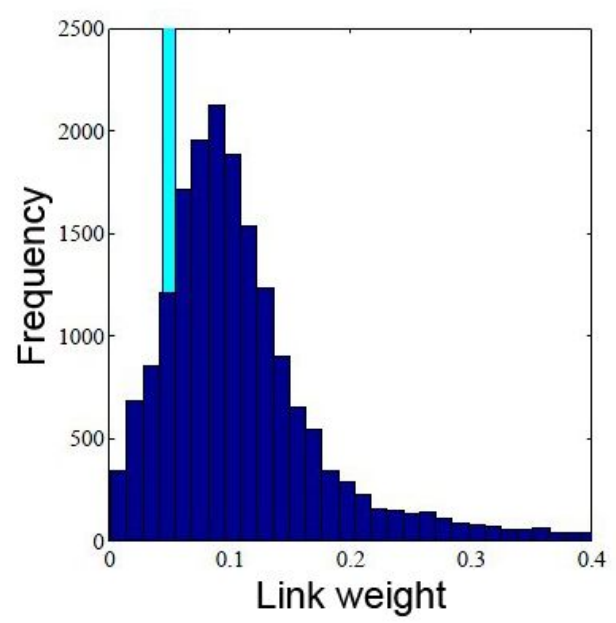

(a)

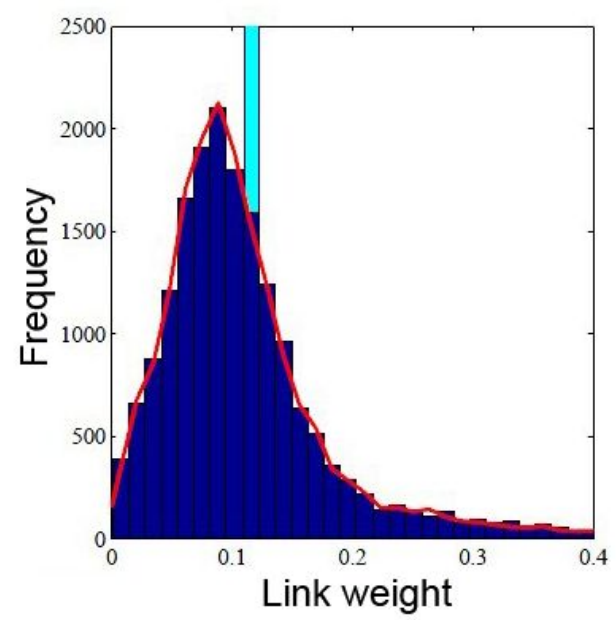

(b)

Figure 3.15. The STDP rule applied to the numerical system. (a) Initial link weight $=0.05$, light blue, and link weight distribution at time 5000. (b) Initial link weight $=0.05$ and link weight distribution at time 5000. System parameters: $t_{l i n k}=1.2, c_{d}=0.019, c_{p}=0.2$.

in which the initial link weight is below the critical threshold for sustained behavior. Provided the system has sufficient time to apply the STDP algorithm, the mean link weight distribution will increase above the critical threshold value needed to sustain behavior. We demonstrate in Fig. 3.15 that this higher mean link weight distribution is an attractor of the system. The simulations that fail to sustain activity correspond to those that collapse prior to the mean link weight exceeding this critical value.

\subsection{Summary}

A chemical network is designed by using the discrete BZ oscillator system. Catalyst-loaded, ion-exchange resin particles are used as the individual elements in the network. The interactions between elements consist of two types of links, similar to the synapse connections in biological 
neural networks. The existence of a steady state and an oscillatory state on each particle offers a model for the spiking process of a neuron. There are many differences between a biological neural network and a BZ-based chemical neural network; however, the fundamental features of a neural network, such as integrate and fire, can be captured by the excitable properties of the BZ oscillators.

In both experiments and numerical simulations, we analyze the network behavior with different numbers of links and develop an analysis of the activity. A learning rule, called STDP, is applied to the training process. The excitable links are updated according the STDP rule and the inhibitory links are maintained at a constant value. Our results show that if the STDP network exhibits sustained activity, a stable distribution of link weights is observed. The stable distribution of link weights is interesting because it is similar to the behavior of a biological network.

The firing events of nodes in the network are complex. The statistics of all firing events show that there is a natural time interval, similar to the refractory period of neuron spike. 


\section{Reference}

[1] C. Henriquez and A. Papazoglou, "Using computer models to understand the roles of tissue structure and membrane dynamics in arrhythmogenesis," Proc. IEEE 84, 334 -354 (1996). Online Version 43

[2] A. L. Hodgkin and A. F. Huxley, "A quantitative description of membrane current and its application to conduction and excitation in nerve," J. Physiol. (Lond.) 117, 500-544 (1952). Online Version 43

[3] N. Wiener and A. Rosenblueth, "The mathematical formulation of the problem of conduction of impulses in a network of connected excitable elements, specifically in cardiac muscle," Arch. Ins. Cardiol. Mex. 16, 205-265 (1946).

Online Version 43

[4] B. G. Farley, "A neural network model and the "slow potentials" of electrophysiology," Computers in Biomedical Res. 1, 265-294 (1965). 43

[5] M. Gerhardt, H. Schuster, and J. Tyson, "A cellular automation model of excitable media including curvature and dispersion," Science 247, 1563-1566 (1990).

Online Version 43

[6] M. Gerhardt, H. Schuster, and J. J. Tyson, "A cellular automaton model of excitable media: II. curvature, dispersion, rotating waves and meandering waves," Physica D 46, 392-415 (1990).

Online Version 43

[7] M. Markus and B. Hess, "Isotropic cellular automaton for modelling excitable media," Nature 
347, 56-58 (1990).

Online Version 43

[8] C. Kurrer and K. Schulten, "Propagation of chemical waves in discrete excitable media: Anisotropic and isotropic wave fronts," in "Nonlinear Wave Processes in Excitable Media, Vol. 244 of NATO Science Series B: Physics," (1990).

Online Version 43

[9] M. Gerhardt, H. Schuster, and J. J. Tyson, "A cellular automaton model of excitable media: III. fitting the Belousov-Zhabotinskii reaction," Physica D 46, 416-426 (1990). Online Version 44

[10] A. Nishiyama, H. Tanaka, and T. Tokihiro, "An isotropic cellular automaton for excitable media," Physica A 387, 3129-3136 (2008).

Online Version

[11] J. R. Weimar, J. J. Tyson, and L. T. Watson, "Diffusion and wave propagation in cellular automaton models of excitable media," Physica D 55, 309-327 (1992).

Online Version 44

[12] R. Toth, A. F. Taylor, and M. R. Tinsley, "Collective behavior of a population of chemically coupled oscillators," J. Phys. Chem. B 110, 10170-10176 (2006).

Online Version 44

[13] A. F. Taylor, P. Kapetanopoulos, B. J. Whitaker, R. Toth, L. Bull, and M. R. Tinsley, "Clusters and switchers in globally coupled photochemical oscillators," Phys. Rev. Lett. 100, 214101-1-4 (2008).

Online Version 46 
[14] R. Rojas, Neural Networks: A Systematic Introduction (Springer, 1996).

Online Version 47

[15] M. C. W. van Rossum, G. Q. Bi, and G. G. Turrigiano, "Stable Hebbian learning from spiketiming-dependent plasticity," J. Neurosci. 20, 8812-8821 (2000).

Online Version 48

[16] C. Zhou, L. Zemanova, G. Zamora, C. C. Hilgetag, and J. Kurths, "Hierarchical organization unveiled by functional connectivity in complex brain networks," Phys. Rev. Lett. 97, 238103$1-4(2006)$.

Online Version 48

[17] H. Berry and O. Temam, "Characterizing self-developing biological neural networks: A first step towards their application to computing systems," in "Lecture Notes in Computer Science," Vol. 3512, 306-317 (2005).

Online Version 48

[18] J. Wang, "Photo-induced excitations in the ruthenium-catalyzed Belousov-Zhabotinsky reaction," Chem. Phys. Lett. 335, 123-126 (2001).

Online Version 54

[19] S. Kádár, T. Amemiya, and K. Showalter, "Reaction mechanism for light sensitivity of the $\mathrm{Ru}(\text { bpy })_{3}^{2+}$-catalyzed Belousov-Zhabotinsky reaction," J. Phys. Chem. A 101, 8200-8206 (1997).

Online Version 58 


\section{Chapter 4}

\section{Phase Kinks in the Belousov-Zhabotinsky}

\section{Reaction}

Synchronization in coupled nonlinear oscillators is a remarkable and ubiquitous phenomenon [1]. Depending on the nature and the strength of the coupling, different synchronization states have been observed, including complete or identical synchronization [2, 3], in-phase [4], out-of-phase $[5,6]$, lag synchronization [7], generalized synchronization [8], intermittent lag synchronization

[9], and mixed synchronization [10]. There has been extensive effort to explore various types of synchronization, including phase synchronization [11].

In phase synchronization, oscillators are correlated in phase but uncorrelated in amplitude. Phase synchronization has been widely studied in recent years, especially in nonlinear chemical reactions, for example, the photosensitive Belousov-Zhabotinsky (BZ) reaction. In this system, the forcing can be introduced through the sensitivity of the reaction to the illumination conditions $[12]$.

In this chapter, the dynamics of a special pattern in the BZ reaction, called a phase kink, is studied under the external forcing. We explore how to trap phase kinks in the oscillatory BZ 


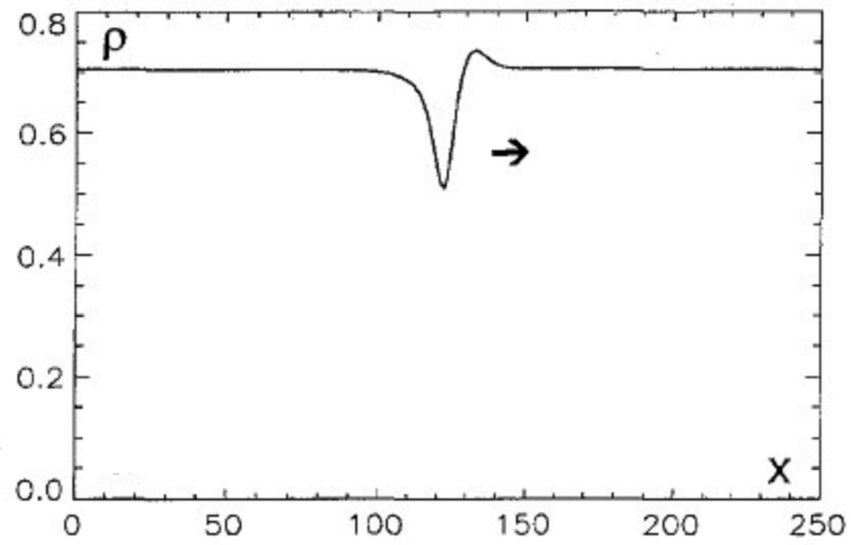

(a)

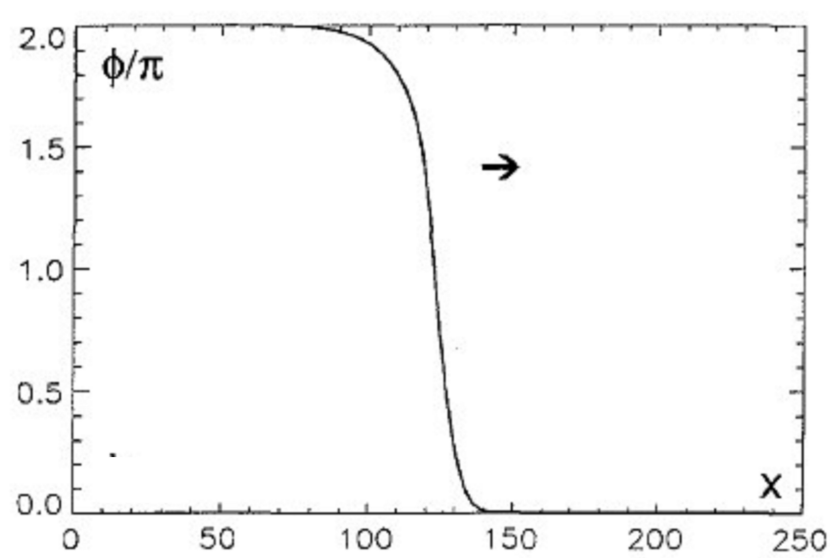

(b)

Figure 4.1. Profiles of the oscillation amplitude modulus (a) and of the phase (b) in traveling phase kinks with global feedback. Figure from reference [14].

reaction.

\subsection{Introduction}

Application of periodic forcing and global feedback to oscillatory media allows us to create new kinds of wave patterns and to control them by variation of the forcing or feedback parameters [12]. Oscillating systems often respond to periodic forcing by adjusting their oscillation frequencies to rational fractions of the forcing frequency. This so-called frequency-locking phenomenon (also called resonance) is accompanied by another significant outcome of the periodic forcing: multiplicity of stable phase states. Each phase state has spatially uniform, frequency-locked oscillations with a fixed oscillation phase [13].

Phase kinks (also called phase flips [14-16] or phase slips [17]) are phenomena that occur through the coupling of nonlinear oscillators via periodic global forcing. The relative phase of phase kinks between the coupled oscillators may jump from zero to $\pi$ or $2 \pi$. However, traveling $\pi$ 


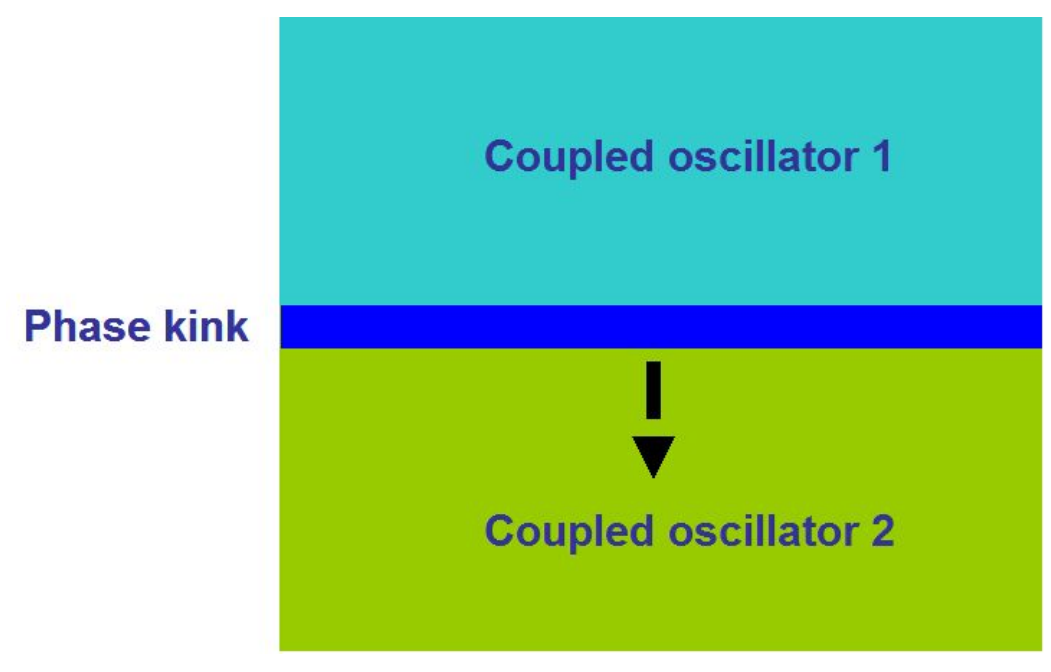

Figure 4.2. In 2D media, phase kink can be created by coupling two oscillators via a third oscillator, such as the periodic global forcing of the media. A phase difference exists between the two coupled oscillators.

fronts under 2:1 forcing are sometimes called Bloch walls [17]. Figure 4.1 shows the phase profile of a steadily traveling $2 \pi$ phase kink.

In $2 \mathrm{D}$ media, kinks correspond to extended traveling patterns where the oscillation phase changes by $\pi$ or $2 \pi$ at a certain line. A schematic diagram of a phase kink in $2 \mathrm{D}$ media is shown in Fig. 4.2. Because the states of the medium are physically identical on both sides of such a pattern, it can be viewed as an analog of traveling waves in 2D excitable media. The difference with respect to traveling excitation waves is that kink patterns may be stationary and can reverse their propagation direction [17].

Kinks in externally forced oscillatory media were first described and investigated by Coullet and Emilsson [18, 19]. When periodic forcing acts on any dynamical system, the effect strongly depends on the relationship between the forcing frequency $f_{1}$ and the natural oscillation frequency $f_{0}$ of the system. When the condition $m f_{1}=n f_{0}$, with integers of $n, m=1,2,3, \ldots$, is realized, the system becomes particularly sensitive to external forcing and resonances are expected. Such 
resonances are conventionally classified through their respective frequency ratios n:m. When the forcing frequency $f_{1}$ coincides with the frequency of natural oscillations $f_{0}(n=m=1)$, a $1: 1$ resonance takes place. Another important parameter, affecting the dynamics of a system with periodic external forcing is the forcing amplitude $A$. Sufficiently strong periodic forcing can entrain a dynamical system even if its frequency does not exactly match a resonant condition [12]. Here, we focus on the resonance with weak forcing. In the entrained state, the system oscillates not at its natural frequency $f_{0}$, but at the resonant frequency imposed by the external forcing. For any fixed amplitude $A$, the entrainment with respect to a particular n:m resonance is only possible if the detuning $\nu$, defined as $\nu=f_{1}-(n / m) f_{0}$, is not too large. This means that, for a particular resonance and a given forcing amplitude $A$, an entrainment window $\nu_{-}(A)<\nu<\nu_{+}(A)$ can be identified, where $\nu_{-}(A)$ and $\nu_{+}(A)$ are the minimum and maximum value of the existing resonance. The entrainment windows grow with the forcing amplitude and, in the parameter plane $(f, A)$ or $(\nu, A)$, they form characteristic structures known as Arnold tongues [12, 20, 21]. Phase kinks have been observed in Arnold tongues with a 1:1 resonance in the chemical reaction of the catalytic reduction of $\mathrm{NO}$ with $\mathrm{CO}$ on a $\mathrm{Pt}(100)$ catalyst [12].

The complex dynamics of phase kinks has been theoretically studied in detail [23]. Three different regimes of kink dynamics depending on the forcing parameters were observed, including regimes for stable stationary kinks, no kink solution and kink-breeding. Figure 4.3 shows the kink dynamics in the three regimes. The regions corresponding to these three regimes are not symmetric inside the Arnold tongue. In regime I, the initial profile evolves toward a stationary wave propagating with a constant velocity. The $2 \pi$ phase kink disappears via the creation of a defect in regime II. In regime III, the initial kink disappears via the occurrence of a defect, but the residual perturbation grows and leads to two new kinks. The process then repeats itself: each of the new kinks disappears, which leads to the creation of two new kinks, etc. The asymptotic 


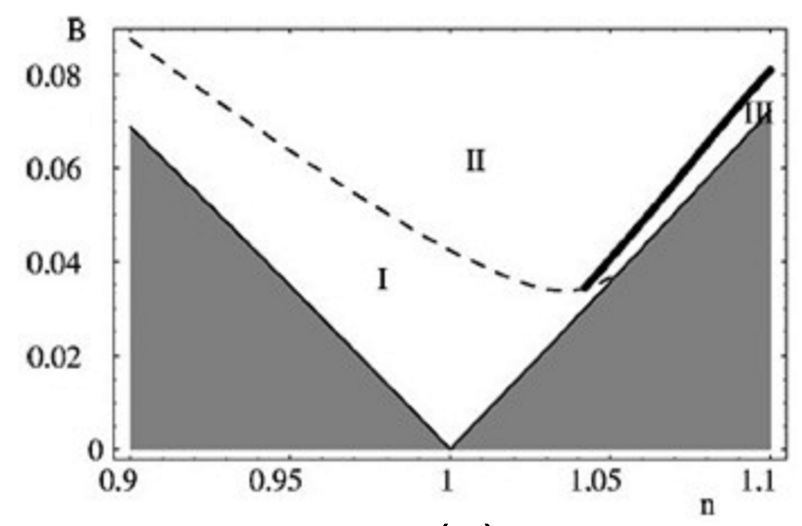

(a)

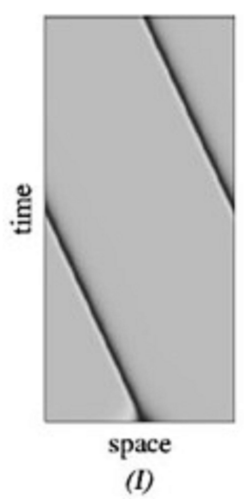

(I)

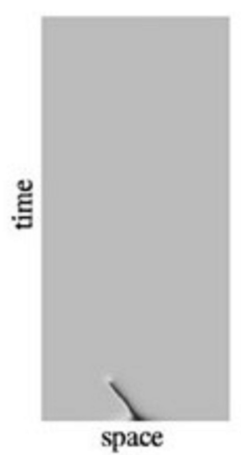

(II)

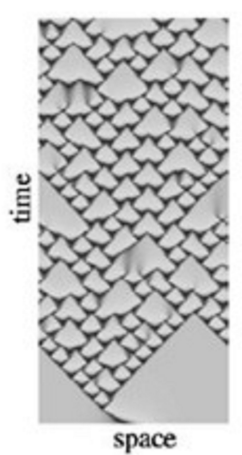

(III)

(b)

Figure 4.3. The complex dynamics of phase kinks in different regions of the Arnold tongue. (a) $B$ is the forcing amplitude and $n$ is the ratio of the forcing frequency to the natural oscillation frequency. The white region represents the Arnold tongue. (b) Space-time plots showing stable stationary kink behavior in Region I, no kink solution in Region II, and kink-breeding behavior in Region III. Figure from reference [22].

spatiotemporal evolution is a disordered state, which is called a back-firing-like instability [22].

The phenomenon of phase kinks occurs in a wide class of systems, such as in chemistry, physics and engineering. The dynamics of phase kinks has been seen in experiments of the catalytic reduction of NO with $\mathrm{CO}$ on a $\mathrm{Pt}(100)$ catalyst [15] and in low frequency fluctuations in a FabryPerot semiconductor laser with optical feedback from an external mirror [15]. More interestingly, a phenomenon called wave traps was observed in the catalytic CO oxidation on platinum [17]. Spatial regions with modified local properties may act as traps where traveling kinks can be stored and released in a controlled way under the 1:1 resonance. In addition, phase synchronized oscillations in neuronal ensembles are also a topic of considerable current interest in neurobiology. For instance, in a recent study of induced focal epilepsy, Li et al. observed both in- and out-of-phase oscillations in spatially separate regions of a rat brain. Since the time-delay in the information transmission 
between different brain regions will depend on their spatial separation, the phase-flip bifurcation may prove useful in understanding the detailed mechanism of dynamical diseases such as epilepsy, or other neurological disorders that are associated with synchronized regions in the brain such as Parkinson's disease [10].

Periodically forcing in time with spatially uniform light has been extensively explored in the oscillatory photosensitive Belousov-Zhabotinsky (BZ) reaction. Specifically, a 2:1 resonance where the system responds at exactly half the forcing frequency has been studied by Lin et al. [20, 21]. They found that the 2:1 resonance exhibits a finite-wave number instability leading to standingwave Turing-like patterns. Pattern dynamics of the oscillatory photosensitive BZ reaction with 1:1 resonance has not been as well explored.

In this chapter, the motive is to experimentally and computationally study the dynamics of phase kinks in the photosensitive oscillatory BZ system. Specially, we are interested in trapping traveling phase kinks in the photosensitive BZ reaction by setting up a region with appropriate amplitude or frequency of external forcing.

\subsection{Experimental Design}

\subsubsection{Experimental Setup}

The experimental setup is shown in Fig. 4.4. It is composed of two components: the chemical reaction and the instrumentation to control and record the spatiotemporal patterns in the photosensitive BZ reaction.

A microscope glass slide bearing a gel, in which the catalyst is immobilized, is placed faceup in a thermostated reactor at $25^{\circ} \mathrm{C}$ and covered by the catalyst-free $\mathrm{BZ}$ solution. This is continuously fed to maintain constant, nonequilibrium conditions [24]. In gel systems [25, 26], there are no 


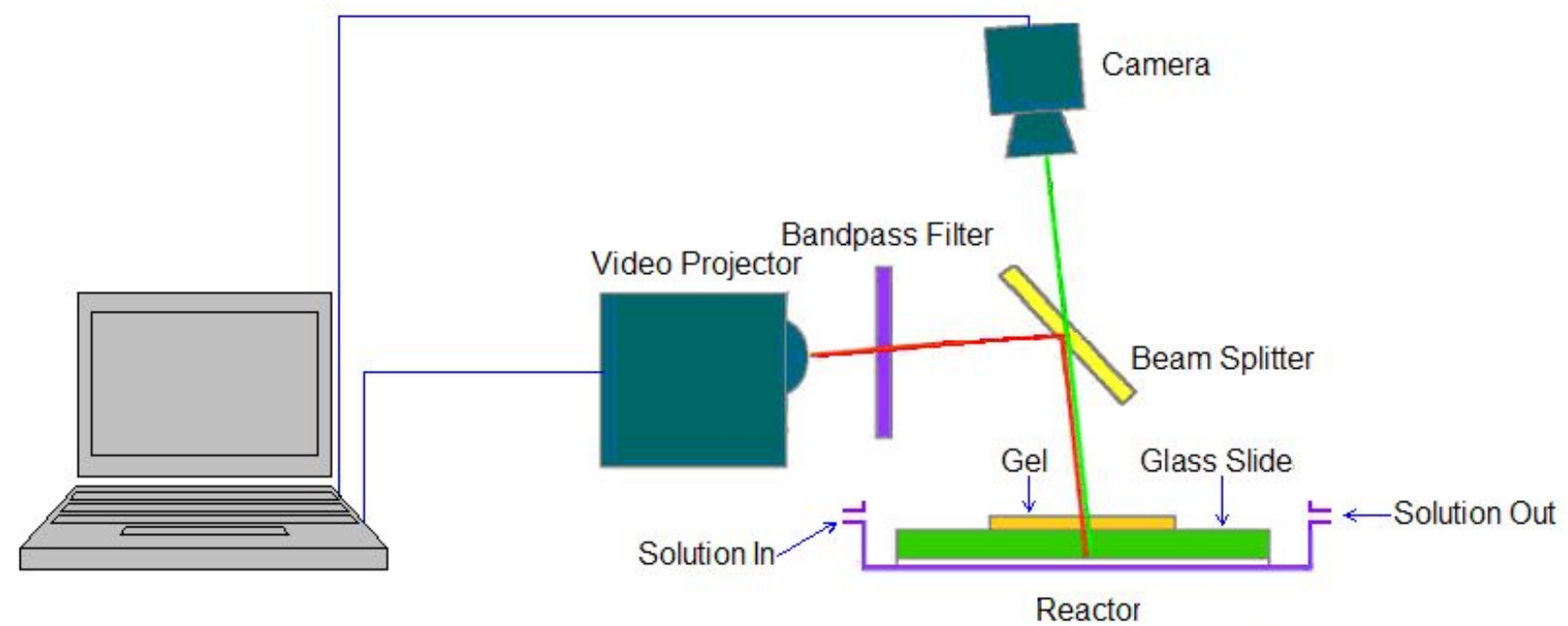

Figure 4.4. The experimental setup for phase kinks in the BZ reaction.

hydrodynamic effects that perturb the spatial pattern, which is essential for the systematic study of chemical waves and patterns. The catalyst-free BZ solution, maintained in an ice bath, is pumped continuously using a peristaltic pump with a fixed flow rate into the reactor through a degasser. The reaction mixture is also pumped out at the same rate so that the depth of the layer of solution in the reactor is kept constant during the experiment.

An illumination profile produced by a computer-controlled, modified video projector is projected onto the gel medium through a $460 \mathrm{~nm}$ bandpass filter and a half-mirror beam splitter. The light from the projector is scattered from a white membrane filter positioned between the glass slide and the bottom of the reactor, and serves as background for observing the chemical patterns. The beam splitter combines the illumination and observation optical paths by matching the illumination and observation angles. The beam from the video projector is mainly reflected down onto the gel. A CCD video camera fitted with a macro lens is used to record the images of the chemical patterns, which are analyzed in real time by the computer for performing the feedback 


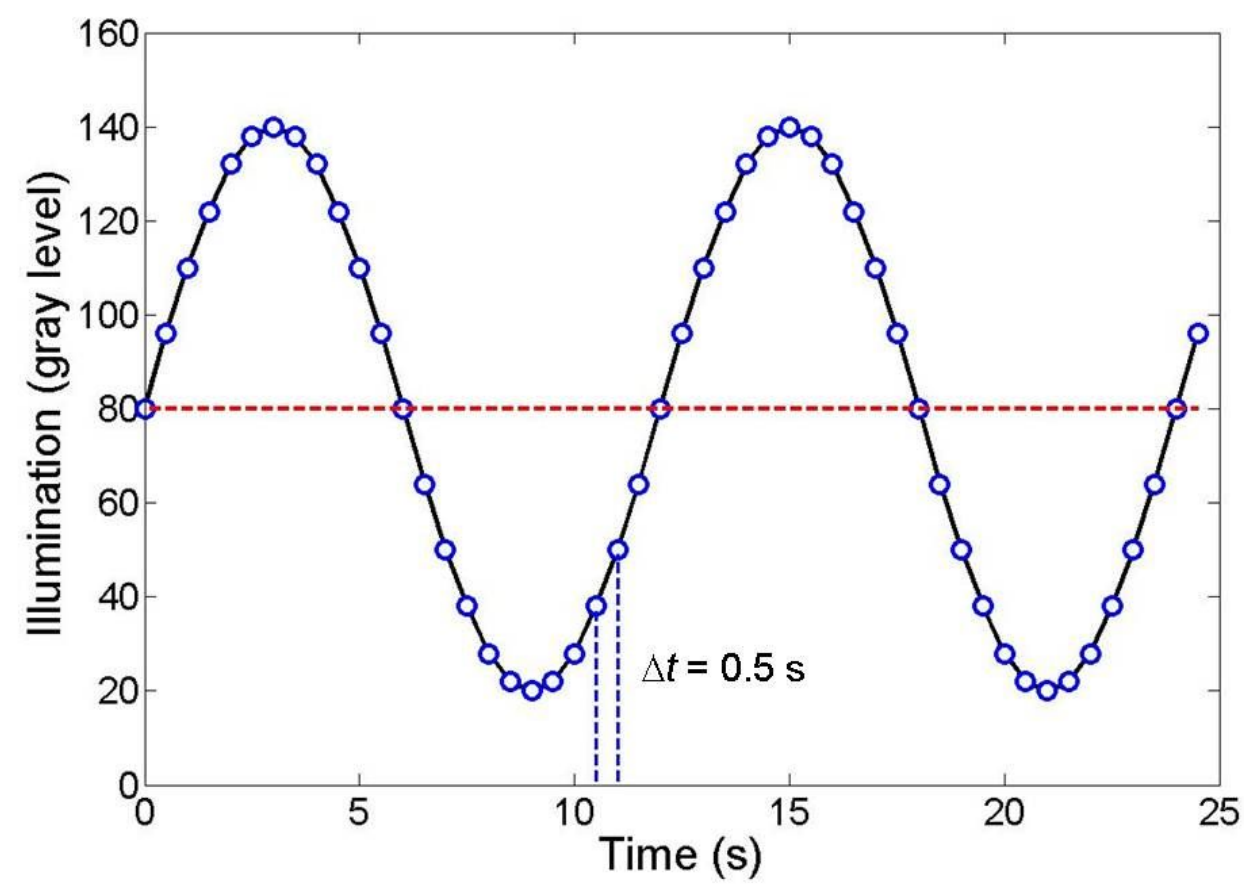

Figure 4.5. A sketch of periodic forcing illumination as a function of time. The illumination $\phi(t)$ is updated according to $\phi(t)=\phi_{0}+\operatorname{Asin}\left(2 \pi f_{1} t\right)$, where $\phi_{0}$ is the background illumination, $A$ is the forcing amplitude, $f_{1}$ is the periodic forcing frequency, and $t=n \Delta t, n=0,1,2 \ldots$ The illumination is updated by modifying the gray level of the projector. In this case, the background illumination with gray level $\phi_{0}=80$, shown as the straight dashed line, is assumed to trigger uniform oscillations in the photosensitive BZ reaction. The amplitude of the forcing illumination $A$ is 60 , and the relative frequency $f_{1}$ is $0.0667 \mathrm{~s}^{-1}$. Every point labeled by (o) represents one forcing illumination event on the reaction medium. In the experiment, light intensities at gray levels of 20,80 and 140 were $0.0705,0.0987$ and $0.2683 \mathrm{~mW} \mathrm{~cm}^{-2}$, respectively. 
profile. The video camera is located above the beam splitter so that its optical axis coincides with the path of the light between the beam splitter and the gel.

When the catalyst $\mathrm{Ru}(\mathrm{bpy})_{3}{ }^{2+}$ is oxidized to $\mathrm{Ru}(\mathrm{bpy})_{3}{ }^{3+}$, its color changes from orange-red to blue-green. The oxidized state, $\mathrm{Ru}(\mathrm{bpy})_{3}{ }^{3+}$, absorbs much less light than the reduced state. The illumination beam passes through the gel to the white membrane filter, scatters, and travels back, to be observed by the camera. Since the distance between the gel and the diffuser is relatively small $(1.0 \mathrm{~mm})$, the profile of the wave in the gel may be seen on the membrane.

Figure 4.5 shows a sketch of periodic forcing by controlling the light illumination with a sine function. The update of periodic forcing is based on varying the gray level of the projector. The forcing of a sine function has been theoretically studied in the reaction-diffusion system [12].

\subsubsection{Preparation of the Chemical System}

Stock solutions of sodium bromide $(\mathrm{NaBr})$, sodium bromate $\left(\mathrm{NaBrO}_{3}\right)$, malonic acid (MA), and sulfuric acid $\left(\mathrm{H}_{2} \mathrm{SO}_{4}\right)$ are prepared with doubly distilled water and kept in the refrigerator at a temperature of $3{ }^{\circ} \mathrm{C}$. Initial concentrations of $\mathrm{NaBr}$ and $\mathrm{MA}$ are $1.0 \mathrm{M}$; initial concentration of $\mathrm{NaBrO}_{3}$ is $2.0 \mathrm{M}$; and initial concentration of $\mathrm{H}_{2} \mathrm{SO}_{4}$ is $3.0 \mathrm{M}$. In experiments, the stock solutions were mixed with appropriate volumes to prepare reaction solutions, where $[\mathrm{MA}]=0.188 \mathrm{M},[\mathrm{NaBr}]$ $=0.108 \mathrm{M},\left[\mathrm{H}_{2} \mathrm{SO}_{4}\right]=0.732 \mathrm{M}$, and $\left[\mathrm{NaBrO}_{3}\right]=0.606 \mathrm{M}$.

The stock solution of $\mathrm{Ru}(\mathrm{bpy})_{3}^{2+}$ is prepared by dissolving the sulfate salt of $\mathrm{Ru}(\mathrm{bpy})_{3}^{2+}$, Tris(2,2'-bipyridyl)dichlororuthenium(II) hexahydrate (Sigma-Aldrich, USA) [27]. An amount of $1.0 \mathrm{~g}(1.34 \mathrm{mmol})$ of $\left[\mathrm{Ru}(\mathrm{bpy})_{3}\right] \mathrm{Cl}_{2} \cdot 6 \mathrm{H}_{2} \mathrm{O}$ is dissolved in $16 \mathrm{~mL}$ of water. Then $40.0 \mathrm{~mL}$ of $5.0 \mathrm{M}$ sulfuric acid is added and the sulfate precipitated. After $24 \mathrm{~h}$ the precipitate is filtered and shed with $96 \%$ ethanol and ether. After drying at $50{ }^{\circ} \mathrm{C}$ for 2 days, the product $\left[\mathrm{Ru}(\mathrm{bpy})_{3}\right] \mathrm{SO}_{4} \cdot \mathrm{H}_{2} \mathrm{O}$ is dissolved in $100.0 \mathrm{~mL}$ of water. The concentration of this stock solution is determined by the 
absorption at 452 and $460 \mathrm{~nm}$ with a UV-VIS spectrophotometer, using the extinction coefficients $\epsilon_{452 \mathrm{~nm}}=11900 \mathrm{~cm}^{-1}$ and $\epsilon_{460 \mathrm{~nm}}=11350 \mathrm{~cm}^{-1}$ at $\mathrm{T}=23.5^{\circ} \mathrm{C}$.

The stock waterglass solution of $\mathrm{SiO}_{2}$ is prepared with solid sodium silicate, which contained $60 \% \mathrm{SiO}_{2}$. The initial concentration of $15 \%(\mathrm{w} / \mathrm{w})$ sodium silicate is prepared by dissolving the appropriate amount of sodium silicate in boiling stirred doubly distilled water. It is boiled for a few minutes and then kept hot while vigorously stirring for about 30 minutes until it is transparent. After cooling to room temperature, water is added to make up for evaporation and the solution is filtered. The catalyst-free BZ solution is freshly prepared before each experiment and is kept in an ice bath from the time of mixing until the end of the experiment [28]. Solutions of $\mathrm{NaBrO}_{3}$, $\mathrm{H}_{2} \mathrm{SO}_{4}$, and $\mathrm{MA}$ are mixed together in the given sequence in a volumetric flask. A computercontrolled syringe pump is used to maintain the desired rate for the addition of $\mathrm{NaBr}$ solution. The solution is homogenized by vigorous mechanical stirring until both the color and the smell of bromine disappears.

An appropriate amount $(2.0-5.0 \mathrm{ml})$ of $15 \%(\mathrm{w} / \mathrm{w})$ sodium silicate solution, $\mathrm{Ru}(\mathrm{bpy})_{3}{ }^{2+}$ so- $^{-}$ lution, and doubly distilled water are well mixed in a clean vial, and $\sim 0.5 \mathrm{ml}$ of $1.2 \mathrm{M} \mathrm{H}_{2} \mathrm{SO}_{4}$ is added while shaking the solution. After mixing the solution vigorously for 2-3 seconds, the mixture is rapidly removed and injected under a glass slide which is supported by two pieces of $0.3 \mathrm{~mm}$ pencil lead on a piece of Plexiglas, where it is allowed to cure. The gel with the glass is removed from the Plexiglas and trimmed to the designed size. The gel is transparent, without any inhomogeneities visible to the naked eye. This preparation of silica gel is an efficient and convenient procedure. The amounts of water and $\mathrm{H}_{2} \mathrm{SO}_{4}$ control the gelation rate, and the amounts of $\mathrm{Na}_{2} \mathrm{SiO}_{3}$ solution and $\mathrm{Ru}(\mathrm{bpy})_{3}{ }^{2+}$ correspond to their concentrations in the experiments. The concentration of ruthenium complex in the gel was $1.80 \pm 0.01 \mathrm{mM}$. The gel size is about $20 \times 20$ $\mathrm{mm}^{2}$ for the observation of oscillations in the experiment. 


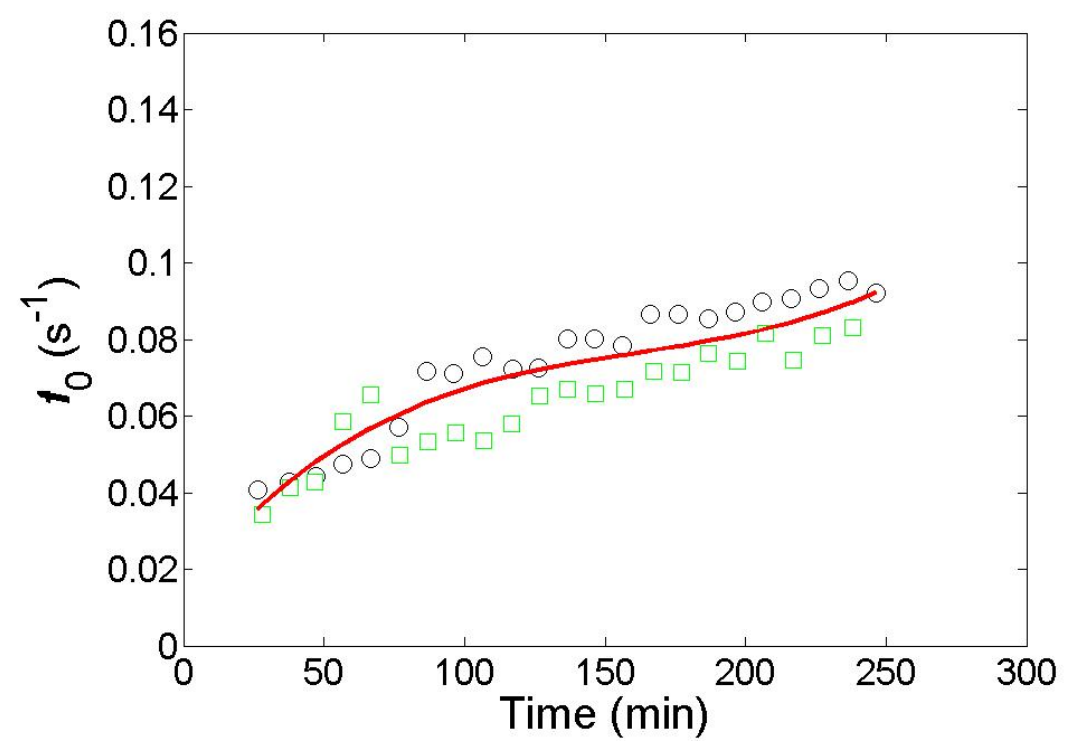

Figure 4.6. The frequency of the background oscillator in the oscillatory BZ reaction as a function of reaction time. Here, the frequency of the background oscillator is calculated every $\sim 10$ minutes. Data were collected from two experiments labeled as $(\mathrm{O})$ and $(\square)$, respectively. The background light intensity is $\phi_{0}=0.0987 \mathrm{~mW} \mathrm{~cm}{ }^{-2}$.

\subsection{Experimental Results}

\subsubsection{The Background Oscillation}

In the experiments, the gel was illuminated with the appropriate intensity so the irradiated gel was in an oscillatory state. After been placed in the reaction solution, the gel began to periodically oscillate after a couple of minutes. In order to obtain a 1:1 frequency-locked resonance, the frequency of external forcing must be reasonably selected according to the natural frequency the oscillators. Thus, the frequency of the background oscillations in the system was monitored first.

Figure 4.6 shows the frequencies of the background oscillations as a function of reaction time. In 
the experiments, the data of oscillations in the gel were collected approximately every ten minutes. The frequency in each time segment was calculated as the reciprocal of the average time interval between sequential oscillations. As shown in Fig. 4.6, the frequency of the oscillations was not constant and increased with time. In order to obtain a frequency-locking forcing, the entrainment of the oscillatory BZ reaction is generally implemented between 100 and 200 minutes, where the frequency change is relatively smooth. As described previously, both frequency and amplitude of external forcing affect the 1:1 frequency-locked entrainment, but we primarily obtained the resonance by adjusting the frequency of the external forcing. Because the frequency of the natural background oscillator changed with time, it was monitored before each forcing and the forcing frequency $f_{1}$ was adjusted according to the newest natural frequency $f_{0}$.

Figure 4.7 shows examples of 1:1 frequency-locked and frequency-unlocked entrainment, where the green dashed lines are the forcing curves and the blue lines are the gray level monitored on the gel surface. In Figure 4.7a, 1:1 frequency-locked behavior is observed. The external forcing entrained the reaction to oscillate the same as its frequency with anti-phase. As shown in Fig. 4.7b, the oscillations on the gel surface became irregular, indicating resonance did not occur. The results show that 1:1 frequency-locked entrainment is feasible by applying an external forcing of a sine function in the oscillatory BZ reaction.

\subsubsection{Creation of Phase Kinks}

Phase kinks can occur with coupled oscillators. Except for the background oscillations, we need at least one more oscillator to create phase kinks in the oscillatory BZ reaction. A pacemaker can serve as the extra oscillator in the reaction. If a medium includes a modified small region where the local oscillation frequency is higher than that of the uniform oscillations, this region can act as

a pacemaker which emits waves forming a target pattern $[29,30]$. In the oscillatory BZ reaction, 


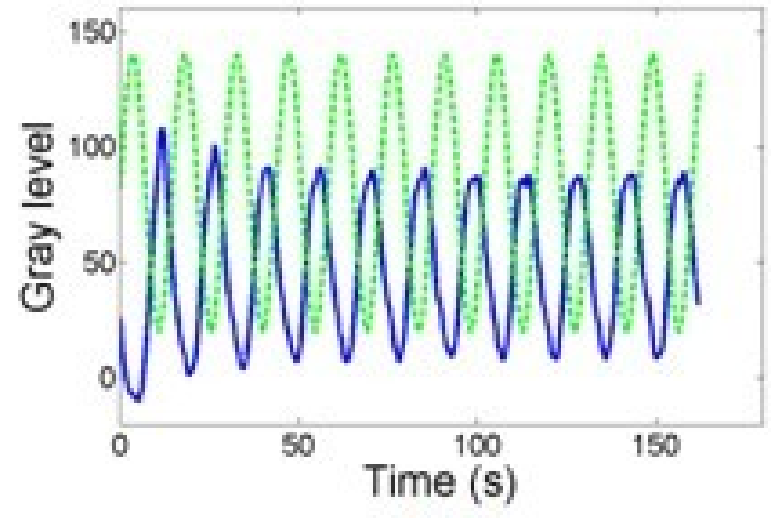

(a)

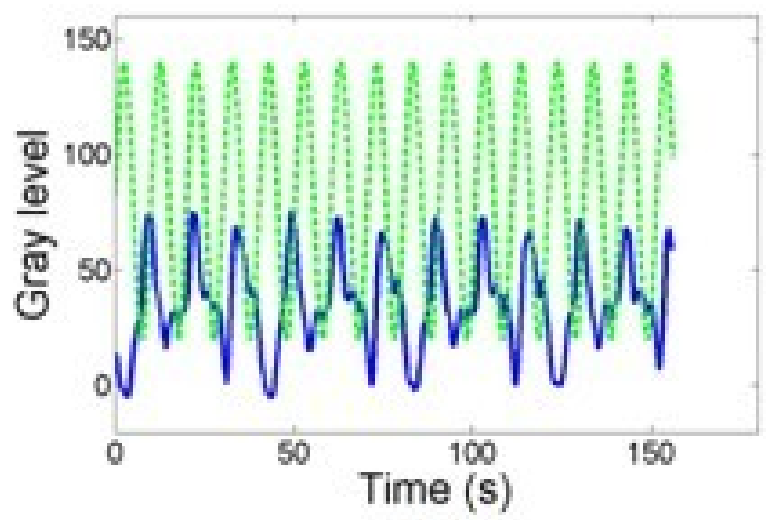

(b)

Figure 4.7. The entrainment of the background oscillations. (a) A 1:1 frequency-locked entrainment. The natural frequency $f_{0}=0.0625 \mathrm{~s}^{-1}$ and the forcing frequency $f_{1}=1.10 f_{0}=0.0687 \mathrm{~s}^{-1}$. (b) Forcing without 1:1 frequency-locked entrainment. The natural frequency $f_{0}=0.0792 s^{-1}$ and the forcing frequency $f_{1}=1.25 f_{0}=0.0990 \mathrm{~s}^{-1}$. The background light intensity $\phi_{0}=0.0987 \mathrm{~mW}$ $\mathrm{cm}^{-2}$; and the periodic amplitude from the projector was 60 gray levels. 
pacemakers arise from heterogeneities, such as dust or gas bubbles. They can also be created by illuminating a specific region with low light intensity.

Pacemakers play an important role in the creation of phase kinks with external forcing. Because the waves emitted by a pacemaker, propagating in the excitable medium, also have the $2 \pi$ phase difference between the front and back, they are the analog of phase kinks. However, phase kinks, represent the existence of a phase difference between coupled oscillators, and their velocity can be affected by adjusting the forcing amplitude and frequency.

In our experiment, one way to create pacemakers in the oscillatory BZ system in a 1:1 frequencylocked resonance is to shield the illumination from the projector so a region (i.e., a corner of the gel) has a lower illumination intensity. In the photosensitive oscillatory BZ reaction, lower illumination intensity creates oscillations with higher frequency. In this way, we can create one more oscillator in the oscillatory BZ reaction. The new oscillator has a higher frequency than the background oscillator. Finally, a wave with $2 \pi$ phase can be observed between these two oscillators. The shielding in the gel is then removed and the region of the pacemaker and the background oscillator are coupled by the external forcing. A single phase kink is thus created as an analog of a propagating wave.

If the shielding is not removed, a series of phase kinks could be created by the pacemakers. For simplicity, only a single kink was studied in our experiments. Thus, as soon as a phase kink occurred, the shielding was removed. Figure 4.8 shows a single phase kink in the 1:1 frequencylocked entrainment in the oscillatory BZ reaction. The phase kink is the whole wave emitted by the pacemaker so the difference between the two sides of the phase kink is $2 \pi$. As shown in Fig. 4.9 , the medium on the two sides of the phase kink oscillates with the same frequencies. The observed speed of the phase kinks in the experiments was $0.06-0.18 \mathrm{~cm} / \mathrm{min}$. 


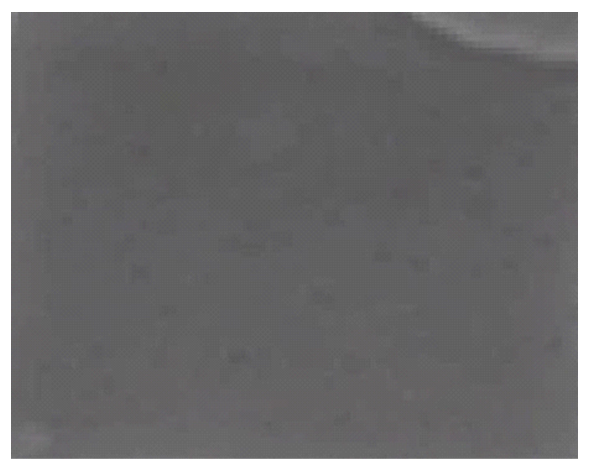

(a)

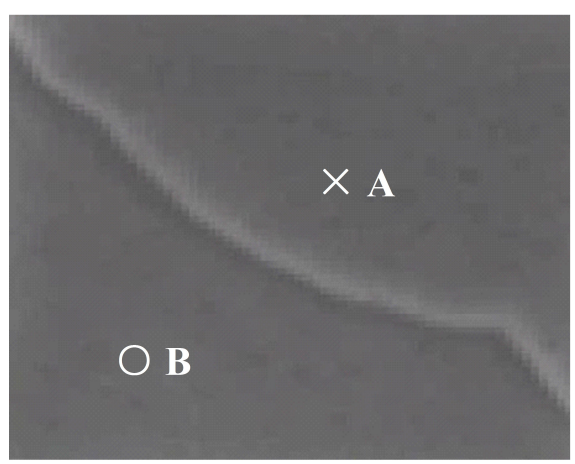

(c)

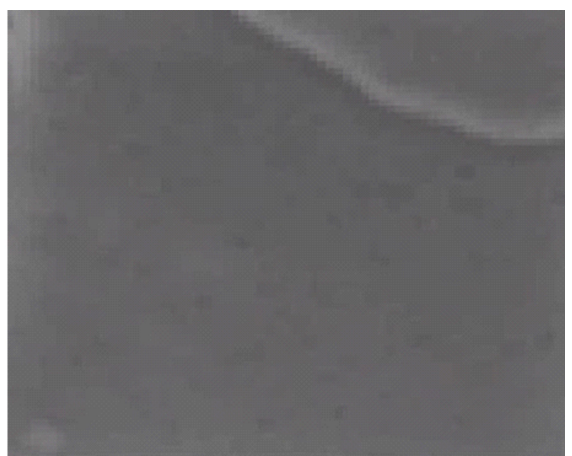

(b)

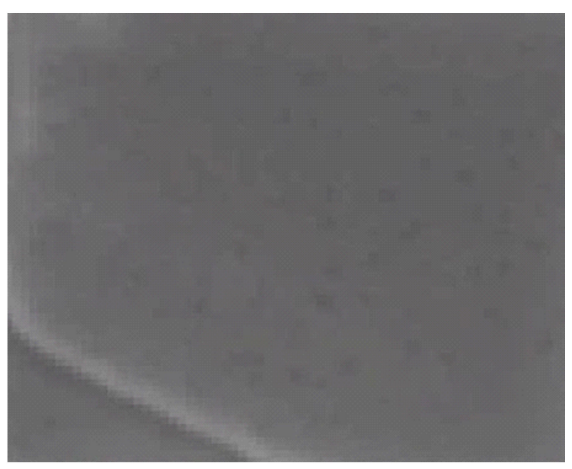

(d)

Figure 4.8. A phase kink propagating in the oscillatory BZ reaction. The phase kink represents the existence of a $2 \pi$ phase difference between two coupled oscillators. In the 1:1 frequency-locked entrainment, the phase kink propagated from the original pacemaker region. The speed of the phase kink was $\sim 0.085 \mathrm{~cm} / \mathrm{min}$. The natural frequency of the background oscillations was $f_{0}=$ $0.0868 \mathrm{~s}^{-1}$. The background light intensity $\phi_{0}=0.0987 \mathrm{~mW} \mathrm{~cm}^{-2}$; the periodic amplitude from the projector was 60 gray levels; the forcing frequency $f_{1}$ is equal to the natural frequency $f_{0}$. 


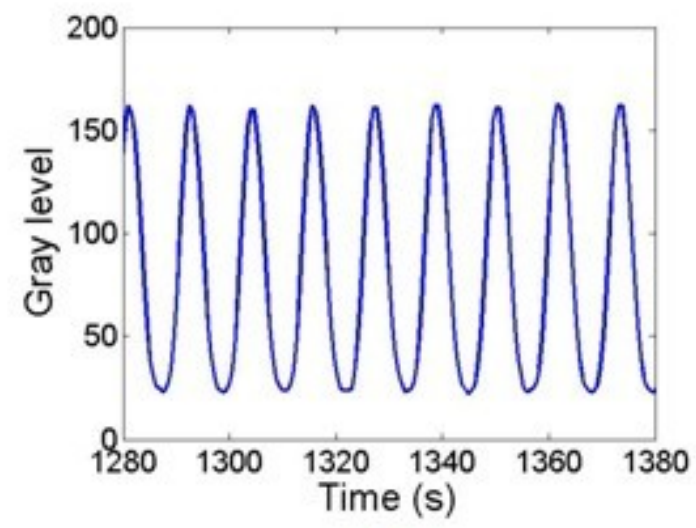

(a)

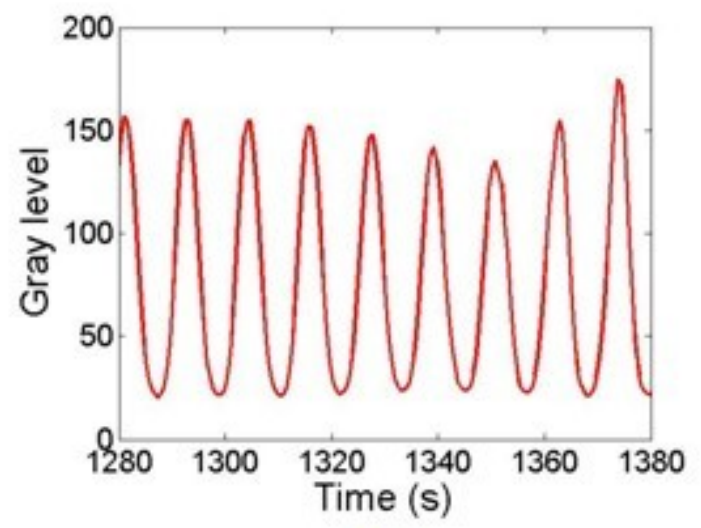

(b)

Figure 4.9. Plots (a) and (b) show the gray level at points A and B in Fig. 4.8, respectively, as a function of time.

\subsubsection{Trap of Phase Kinks}

It has been theoretically verified that the velocity of a phase kink can be changed by controlling the forcing conditions. The propagation direction of a phase kink can even be reversed for specific parameter values. In coupled oscillatory media, kinks propagate from their origin to the periphery of the medium, with a speed that is independent of the generation frequency, if the kink velocity $V$ is positive. This yields a sparse pattern of kinks, running away from a pacemaker. The situation is different if the kink propagation is reversed with $V<0$. In this case, the supercritical inhomogeneity forming the core of the pacemaker generates $2 \pi$ phase kinks which, instead of propagating away as single kinks, form a cluster surrounding the core. Since further phase kinks are continuously produced and added to this cluster, it slowly grows with time. As a result, a pattern that can be viewed as an aggregation of kinks is formed. This principle allows the design of a phase kink trap in oscillatory systems. Recently, Rudzick et al. [17] have successfully trapped traveling $2 \pi$ phase kinks in the reaction of the catalytic reduction of $\mathrm{NO}$ with $\mathrm{CO}$ on a $\mathrm{Pt}(100)$ catalyst. Inspired by 


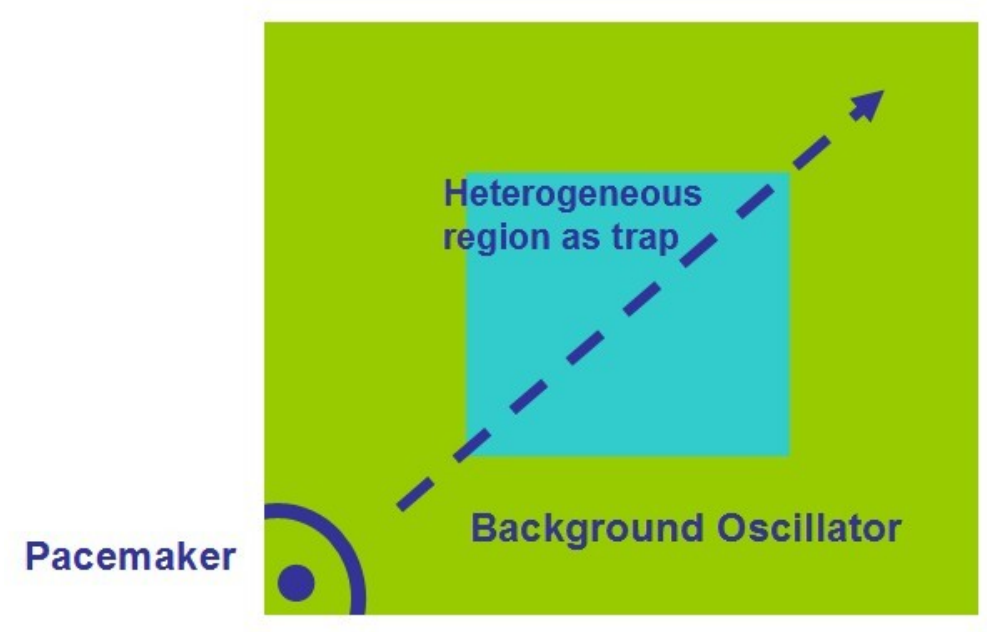

Figure 4.10. Schematic diagram for trapping a phase kink in the oscillatory BZ reaction. The background oscillator and a pacemaker are coupled with the 1:1 frequency-locked entrainment. The heterogeneous region for the trap is entrained with a different external periodic forcing.

their work, we have studied trapping phase kinks in the oscillatory BZ reaction.

Figure 4.10 shows a schematic diagram for trapping a phase kink in the oscillatory BZ reaction. In the 1:1 frequency-locked resonance, a phase kink is created in a corner of the gel and propagates in the medium. A specific trap region with different forcing conditions is designed in the middle of the gel. In this design, a phase kink has the longest propagating distance before it passes out of the medium. We expect that a trap region with appropriate settings can decrease the speed of a phase kink and finally form a closed pattern with a constant phase difference.

Figure 4.11 shows the process of trapping a single phase kink in the oscillatory BZ reaction. The phase kink was generated in the the top-left corner by the coupling of a pacemaker and the background oscillator with 1:1 frequency-locked entrainment. A specific trapping region had been induced in the middle by adjusting the forcing amplitude and frequency. Figure 4.11a-c shows that the phase kink travels with constant speed outside the trap region. Figure 4.11d shows that 


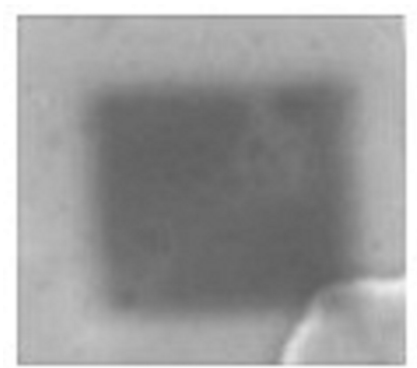

(a)

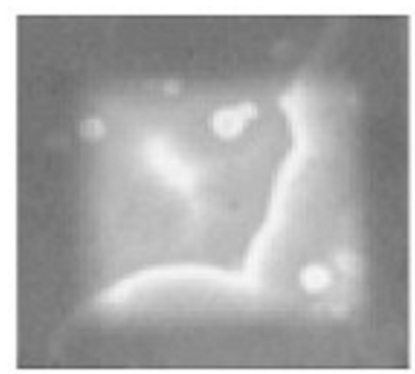

(d)

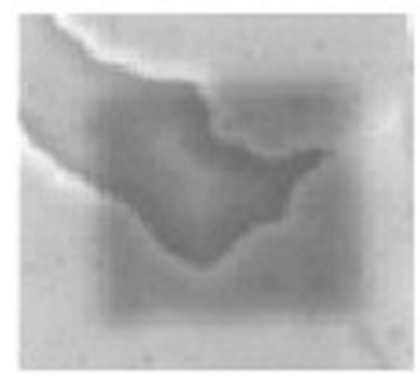

(g)

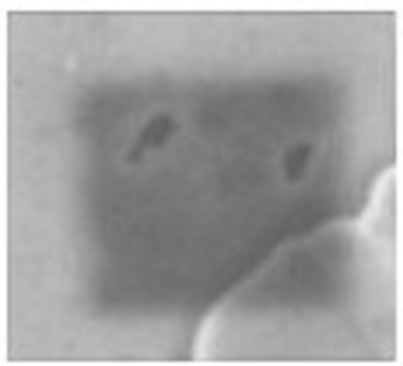

(b)

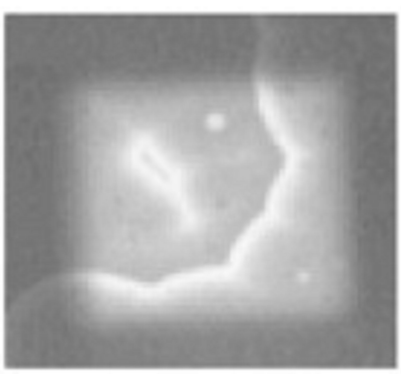

(e)

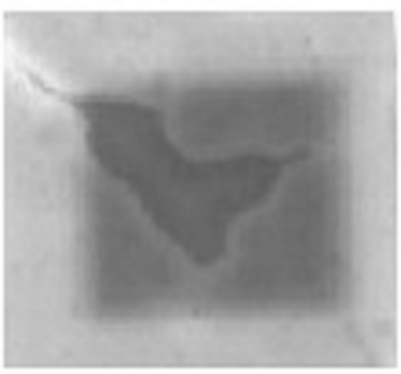

(h)

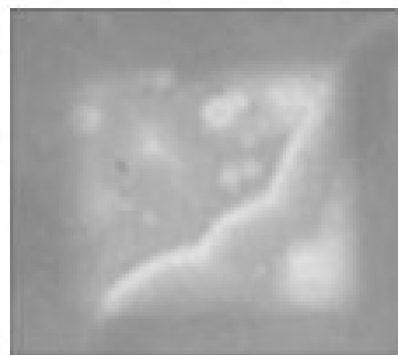

(c)

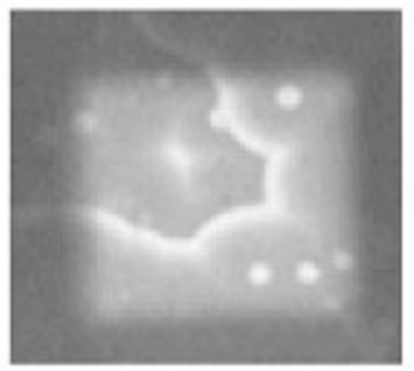

(f)

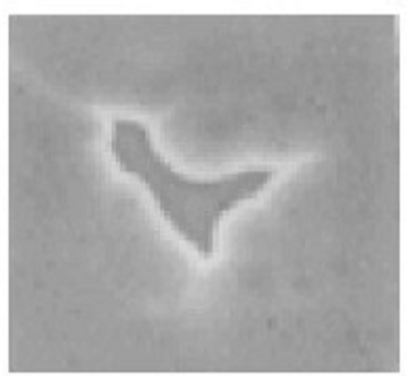

(i)

Figure 4.11. Snapshots of trapping a phase kink in the oscillatory BZ reaction. In the 1:1 frequency-locked entrainment, a phase kink was generated in the top-left corner, and propagated along the diagonal. The middle region was induced as a trap with the different forcing. The natural frequency of the background oscillations was $0.0645 \mathrm{~s}^{-1}$. Experimental conditions: the background light intensity $\phi_{0}=0.0987 \mathrm{~mW} \mathrm{~cm}^{-2}$ (80 gray levels); outside the trap region, the periodic amplitude from projector was 60 gray levels and the forcing frequency $f_{1}=1.03 f_{0}=$ $0.0665 \mathrm{~s}^{-1}$; inside the trap region, the periodic amplitude from projector was 20 gray levels and the forcing frequency $f_{1}=1.13 f_{0}=0.0729 \mathrm{~s}^{-1}$. 
the shape of the phase kink becomes curved as soon as it enters the trapping region. In Figure 4.11e-g, the speed of different segments in the phase kink are clearly different. Because the middle segment first enters the trap region, the effect of trap region on its speed is the largest and it has the lowest propagating speed. The two sides propagate faster than the middle so the phase kink keeps bending. In Figure 4.11h-i, the phase kink finally forms a closed irregular rectangle. During this process, a reversal in direction of the phase kink should occur, although reversal was very short before forming the closed pattern with a constant phase difference. The final closed pattern is irregular because the nonuniform medium results in the segments of the phase kink propagating with different speeds inside and outside the trap region.

The trapping process is affected by both of the resonance parameters and the size of the trap region. Figure 4.12 shows an unsuccessful trapping process regarding the size of the trap region. In this setting, the decrease of speed for the segment of the phase kink in the trap region is so small that the two sides of the phase kink can not bend completely during the process of propagating through the trap region. Theoretical investigations show that the dynamics of phase kinks are different depending on the position in the Arnold tongue. This means that some experiments may favor the occurrence of trapping phenomena and others do not. Because the frequency of the background oscillation is varying, it is difficult to entrain the gel in exactly the same dynamic state with respect to the Arnold tongue.

Pacemakers, generated due to the natural heterogeneities in the medium, present experimental difficulties. In fact, since such heterogeneities are present in large numbers in real systems, oscillatory media becomes naturally populated with pacemakers and, instead of uniform oscillations, multiple target patterns are observed. Even slight heterogeneities in the oscillatory BZ reaction, such as dust or gas bubbles, lead to the development of pacemakers. The effect of pacemakers is shown in Fig. 4.13. The pacemakers might occur inside or outside the trap region as shown in 


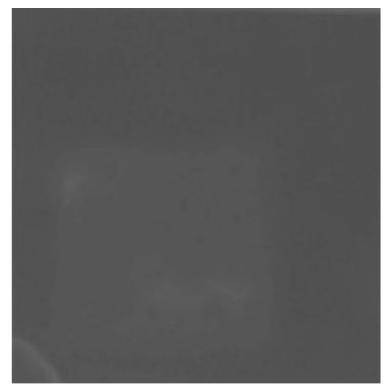

(a)

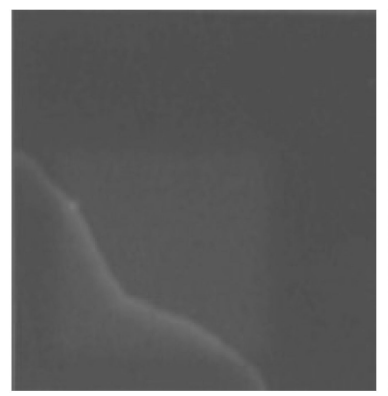

(d)

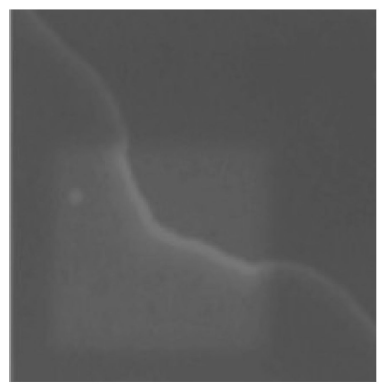

(g)

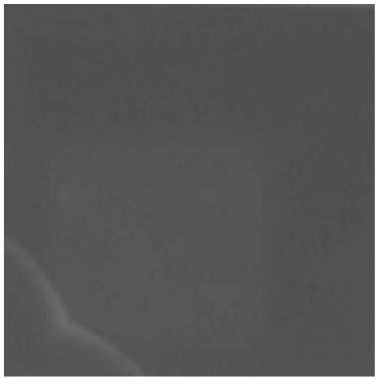

(b)

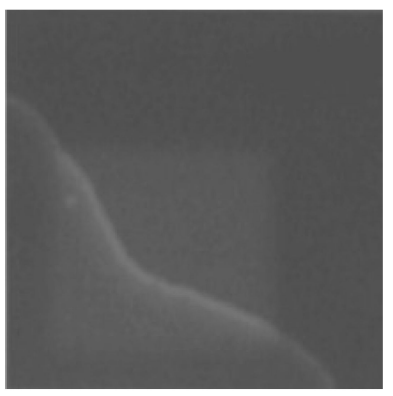

(e)

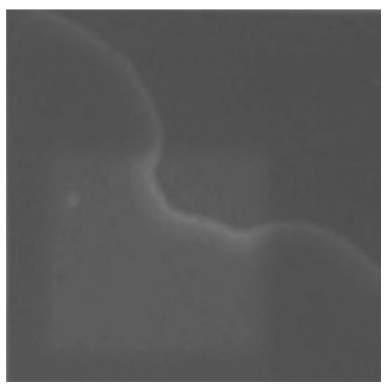

(h)

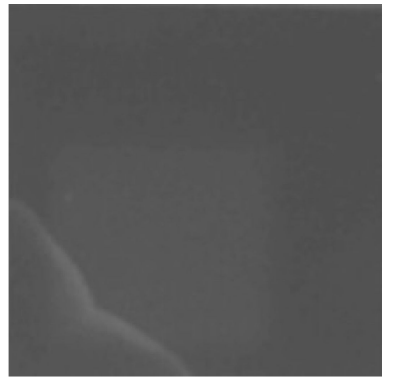

(c)

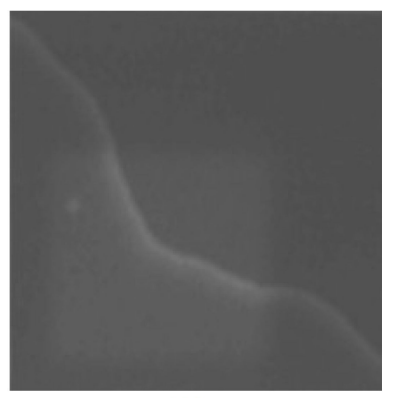

(f)

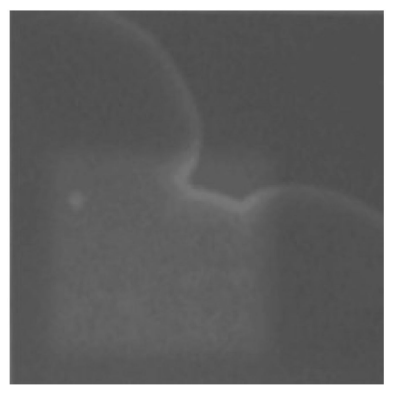

(i)

Figure 4.12. An unsuccessful trapping of a phase kink in the oscillatory BZ reaction. In the 1:1 frequency-locked entrainment, a phase kink was generated in the bottom-left corner, and propagated along the diagonal. The middle region was induced as the trap with different forcing. The natural frequency of the background oscillations was $0.0773 \mathrm{~s}^{-1}$. Experimental conditions: the background light intensity $\phi_{0}=0.0987 \mathrm{~mW} \mathrm{~cm}^{-2}$ (80 gray levels); outside the trap region, the periodic amplitude from the projector was 60 gray levels and the forcing frequency $f_{1}=1.03 f_{0}$ $=0.0797 \mathrm{~s}^{-1}$; inside the trap region, the periodic amplitude from the projector was 20 gray levels and the forcing frequency $f_{1}=1.196 f_{0}=0.0924 \mathrm{~s}^{-1}$. 


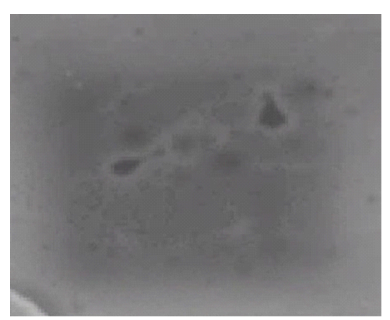

(a)

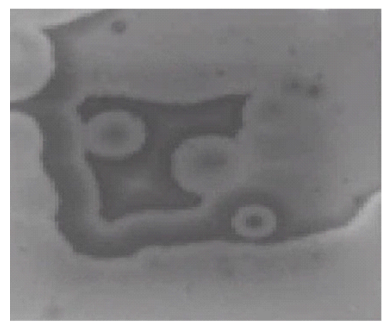

(d)

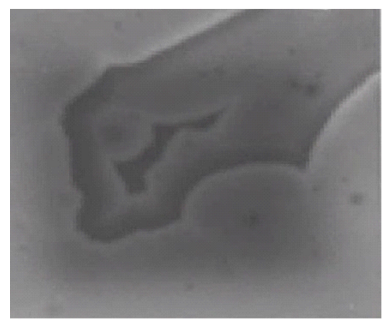

(g)

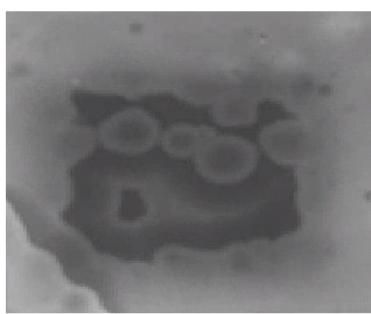

(b)

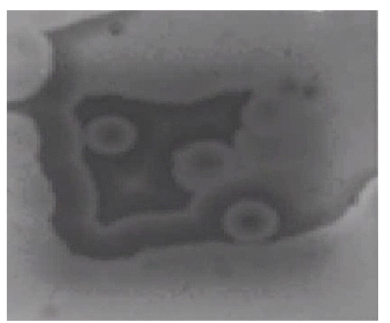

(e)

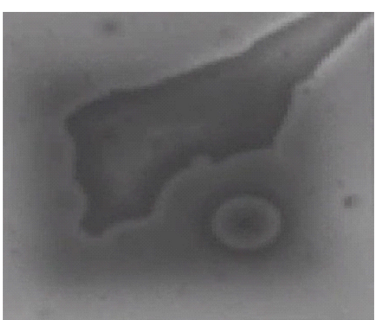

(h)

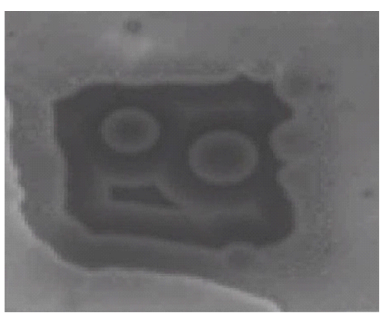

(c)

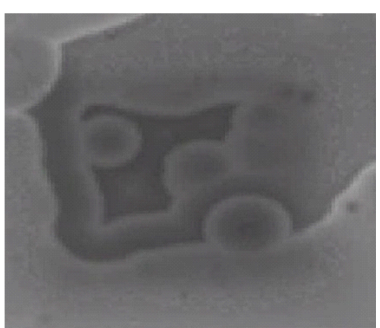

(f)

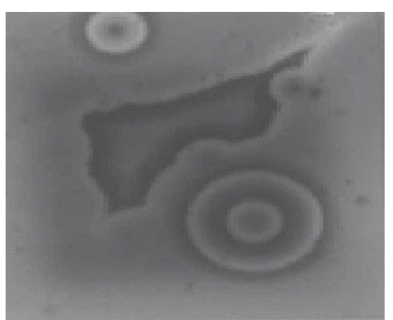

(i)

Figure 4.13. Disturbance of pacemakers in a process of trapping a phase kink in the oscillatory BZ reaction. In the 1:1 frequency-locked entrainment, a phase kink was generated in the bottomleft corner, and propagated along the diagonal. The middle region was induced as a trap with a different forcing frequency and amplitude. The natural frequency of the background oscillations was $0.0773 \mathrm{~s}^{-1}$. Experimental conditions: the background light intensity $\phi_{0}=0.0987 \mathrm{~mW} \mathrm{~cm}^{-2}$ (80 gray levels); outside the trap region, the periodic amplitude from the projector was 60 gray levels and the forcing frequency $f_{1}=1.03 f_{0}=0.0723 \mathrm{~s}^{-1}$; inside the trap region, the periodic amplitude from the projector was 20 gray levels and the forcing frequency $f_{1}=1.204 f_{0}=0.0846$ $\mathrm{S}^{-1}$ 
Fig. 4.13b-e, h and i. The pacemakers might disappear during the forcing process. In addition, different phase kinks can combine to form a pattern when encountering each other, as shown in Fig. 4.13e-f. In Figure 4.13h-i, a closed pattern with phase differences was formed by the existence of newly formed pacemakers.

\subsection{Simulations}

We can study the dynamics of phase kinks in the BZ reaction using the photosensitive Oregonator model below

$$
\begin{aligned}
& \frac{\partial u}{\partial t}=D_{u} \nabla^{2} u+\frac{1}{\epsilon}\left\{u-u^{2}-[\phi+f v] \frac{u-q}{u+q}\right\}, \\
& \frac{\partial v}{\partial t}=u-v .
\end{aligned}
$$

where $u$ and $v$ are the dimensionless concentrations of $\mathrm{HBrO}_{2}$ and $\mathrm{Ru}(\mathrm{bpy})^{3+}$ on the gel, and $\phi$ represents the rate of bromide production due to the irradiation [31].

Figure 4.14 shows a phase kink in the photosensitive Oregonator model. The medium is set in the oscillatory state. A pacemaker is created by lowering the parameter $\phi$. As soon as a wave has been emitted from the pacemaker, periodic forcing is applied to the medium. In the 1:1 frequency-locked entrainment, a phase kink is created and propagates much like a wave emitted from a pacemaker in an excitable medium.

In the 1:1 frequency-locked medium, the region of the original pacemaker is entrained to oscillate with the frequency of the external forcing. Figure 4.15 shows that the evolution of the oscillator in the location of the original pacemaker. Although the local heterogeneities are removed, further phase kinks are continuously produced from the local heterogeneities in the external forcing. As shown in Fig. 4.15, the area of the coupled oscillator from the location of the original pacemaker 


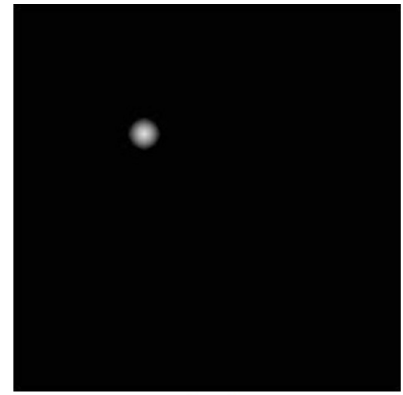

(a)

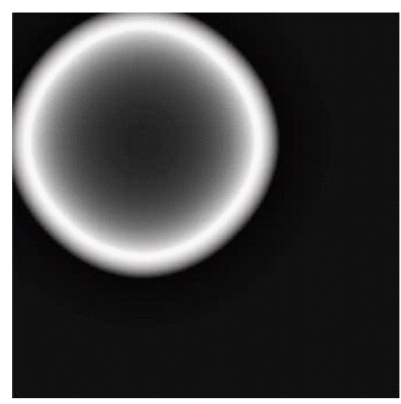

(d)

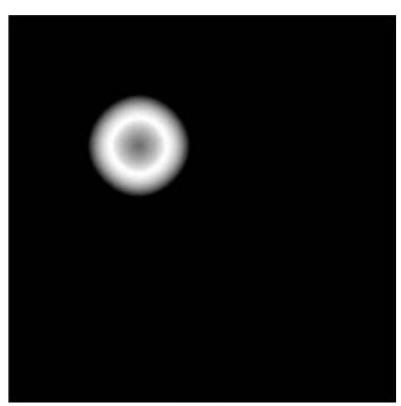

(b)

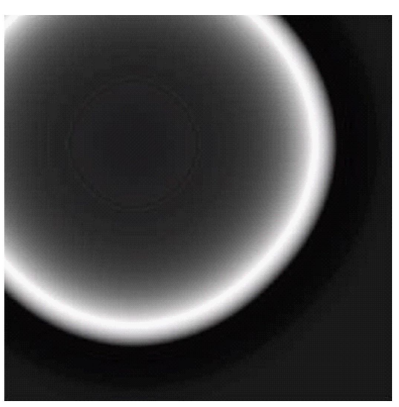

(e)

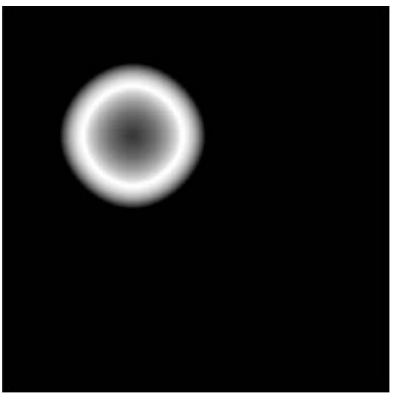

(c)

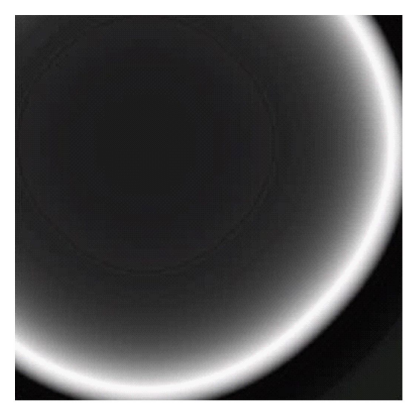

(f)

Figure 4.14. Snapshots of a phase kink calculated using the photosensitive Oregonator model. Simulation conditions: the numerical size $=1000 \times 1000, d x=0.15, d t=0.002, q=0.001, \epsilon=$ 0.005, $f=0.7$, and $D_{u}=1.0$. The step number of the simulation $=1 \times 10^{5}$. The background illuminaition $\phi_{0}=0.003$, the forcing amplitude $\phi_{1}=0.002$, and the forcing frequency $f_{1}$ is equal to the natural frequency $f_{0}=0.1667$. The pacemaker is created by initially using $\phi=0.001$ in a specific small region. 
becomes larger and larger during the propagation of the phase kink.

Figure 4.16 shows the change of a phase kink amplitude during a period. The observed scale is a segment of the diagonal beginning from the original pacemaker. Figure 4.16a corresponds to a status similar to Fig. 4.15f, where a whole wave can be observed. The coupled oscillators then begin to oscillate and the changes in the phase kink are shown in Fig. 4.16b-h. After a period of resonance, a whole wave can again be observed. Figure 4.16a-i suggests that the phase kink has propagated a distance because the location of peak in Fig. 4.16i is greater than that in Fig. 4.16a. As shown in Fig. 4.16, at any time, the phase difference remains $2 \pi$.

An Arnold tongue represents the region in the $f, A$ plane corresponding to the synchronized state of the oscillators. Outside the Arnold tongue, the frequency of the oscillations in a medium can not be entrained at the forcing frequency. Figure 4.17 shows an Arnold tongue for 1:1 frequency-locked entrainment using the photosensitive Oregonator model. As shown in Fig. 4.17, the shape of the Arnold tongue is appropriately symmetric around the natural frequency $f_{0}$ when the amplitude of the external forcing is smaller than 0.00015 . When the amplitude of external forcing increases to 0.0002, the Arnold tongue become asymmetric. A similar asymmetry of the Arnold tongue has been observed in other systems and entrainment phenomena [12, 20, 21]. Figure 4.18 shows the effect of the amplitude and frequency of the periodic external forcing on the speed of a phase kink. As shown in Fig. 4.18a, when the forcing amplitude is small and the forcing frequency $f_{1}$ is the same as the natural frequency $f_{0}$, its effect on the speed of the phase kink is linear. The effect of forcing frequency is also studied. The dynamics of the phase kinks generated in different regime of the Arnold tongue are different. Theoretical investigations have shown that there are at least three regimes with different complex dynamics of phase kinks $[12,22]$. It also suggests that the reversal of the propagation direction of a phase kink only occurs in the regime with forcing frequency $f_{1}$ greater than natural frequency $f_{0}[17]$. Thus, we are more 


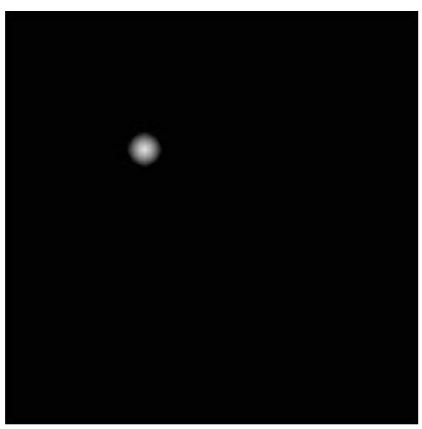

(a)

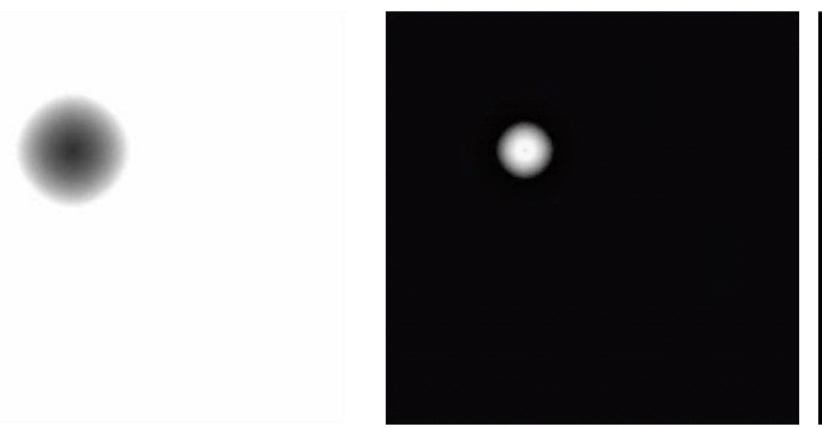

(d)

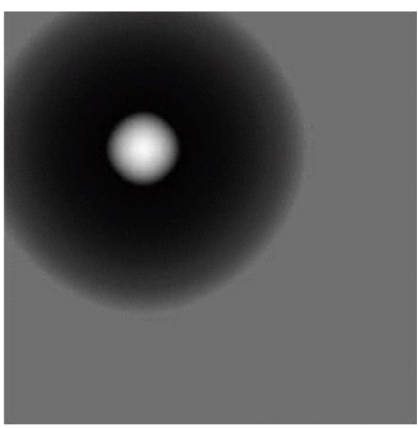

(g)

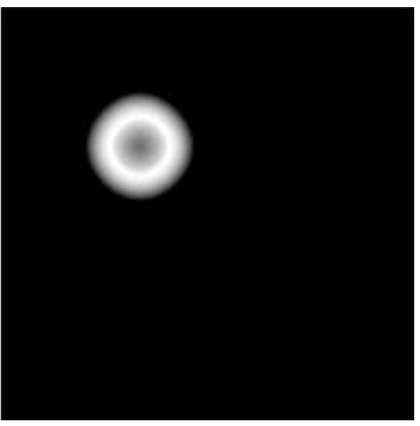

(b)

(e)

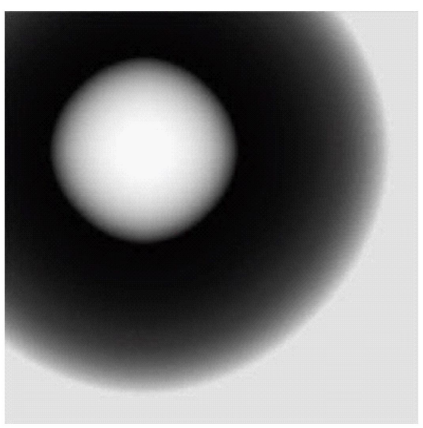

(h)

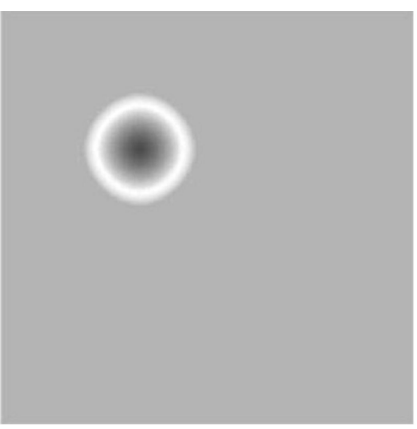

(c)

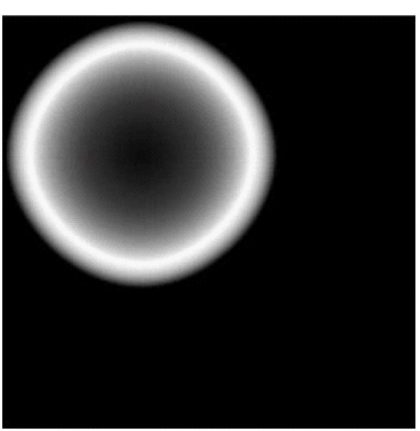

(f)

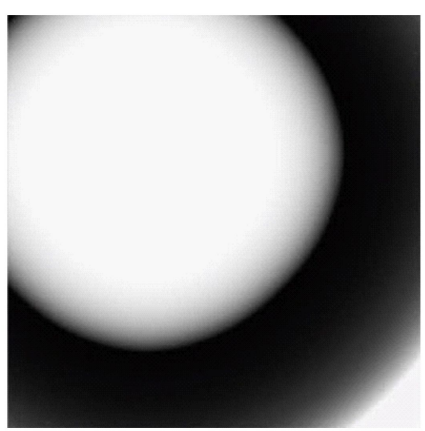

(i)

Figure 4.15. Collapse of the local heterogeneity in the photosensitive Oregonator model with external forcing. Simulation conditions are the same as in Fig. 4.14. 


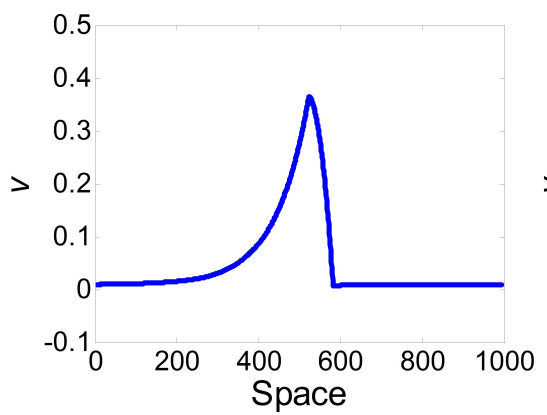

(a)

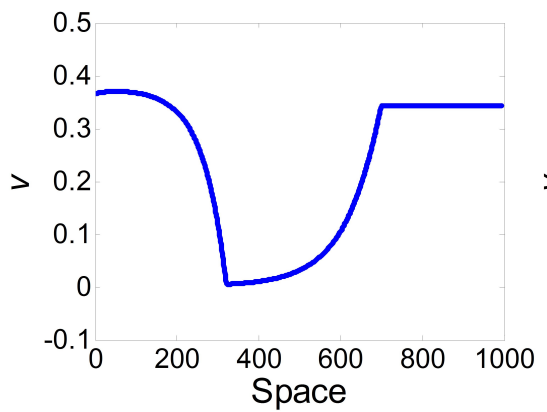

(d)

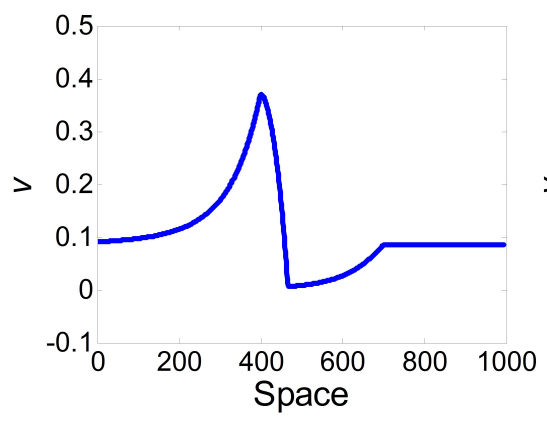

(g)

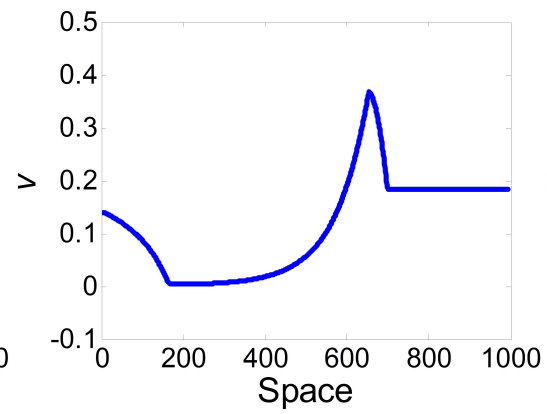

(b)

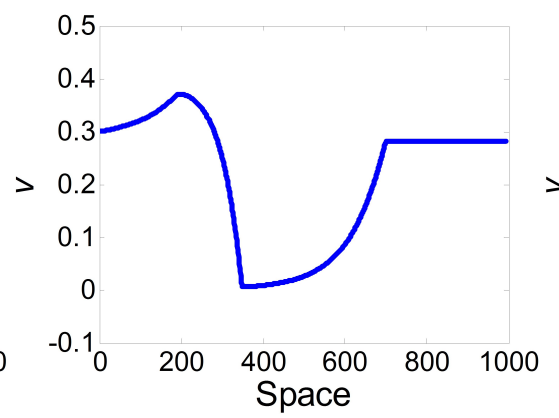

(e)

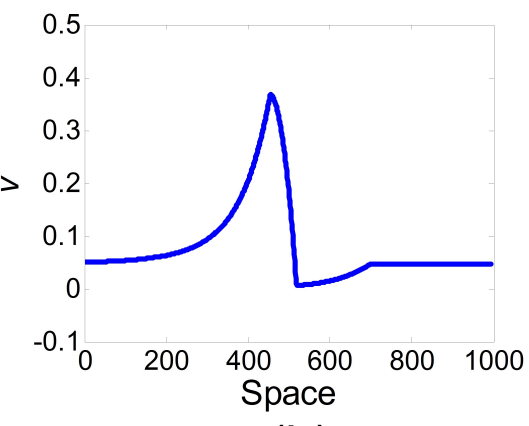

(h)

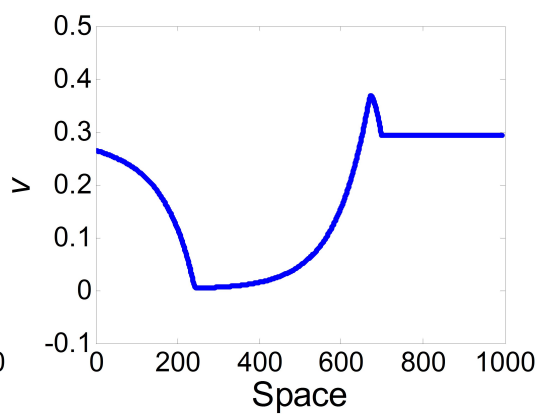

(c)

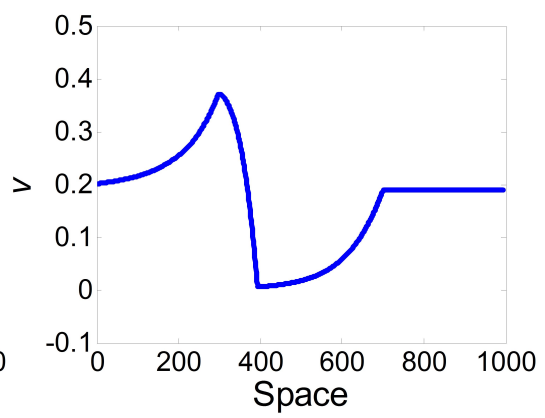

(f)

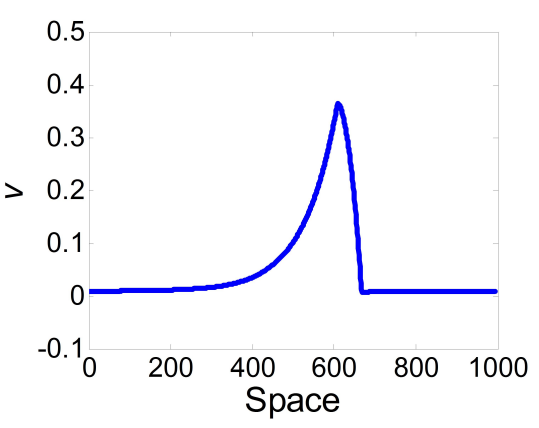

(i)

Figure 4.16. Amplitude change of phase kink during a period. Simulation conditions are the same as in Fig. 4.15 . 


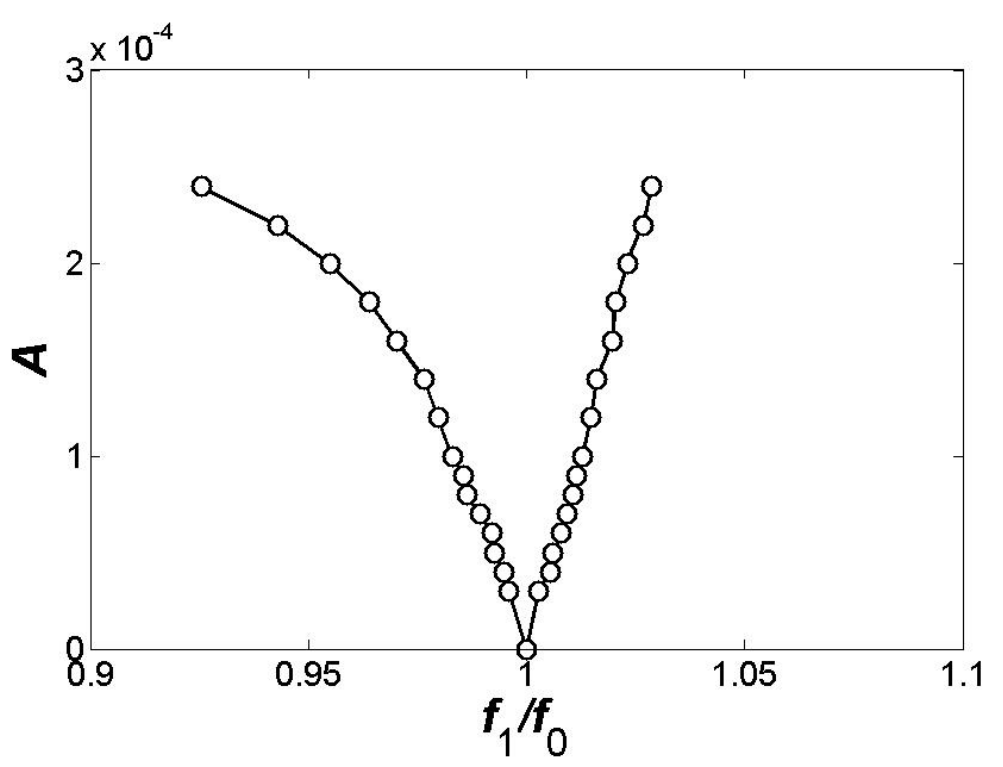

Figure 4.17. Arnold tongue with 1:1 frequency-locked entrainment in the photosensitive Oregonator model. Simulation conditions: $d x=0.15, d t=0.004, q=0.002, \epsilon=0.05, f=2.1$, and $D_{u}$ $=1.0$. The background illuminaition $\phi_{0}=0.0005$, and the natural frequency $f_{0}=0.1662$.

interested in the effect of forcing frequency $f_{1}$ in such regimes, where it is greater than the natural frequency $f_{0}$. Figure $4.18 \mathrm{~b}$ shows the effect of forcing frequency $f_{1}$ on the speed of a phase kink with a forcing amplitude of 0.0002 . The speeds of the phase kink decrease monotonically when the forcing frequencies are a little greater than the natural frequency $f_{0}$. Unfortunately, the negative speed of a phase kink by adjusting the forcing frequency $f_{1}$ corresponding to the amplitude of 0.0002 has been not observed.

However, as shown in Fig. 4.19, a trap simulation using the photosensitive Oregonator model has been observed by adjusting both the amplitude and frequency of periodic forcing in the trap region. In the simulation, a phase kink is created from the top-left corner, and it propagates along the diagonal. As it enters the trap region, the relative segment propagates with a smaller speed, shown in Fig. 4.19b-c. In Figure 4.19d-e, the reversal of the propagation direction of the phase 


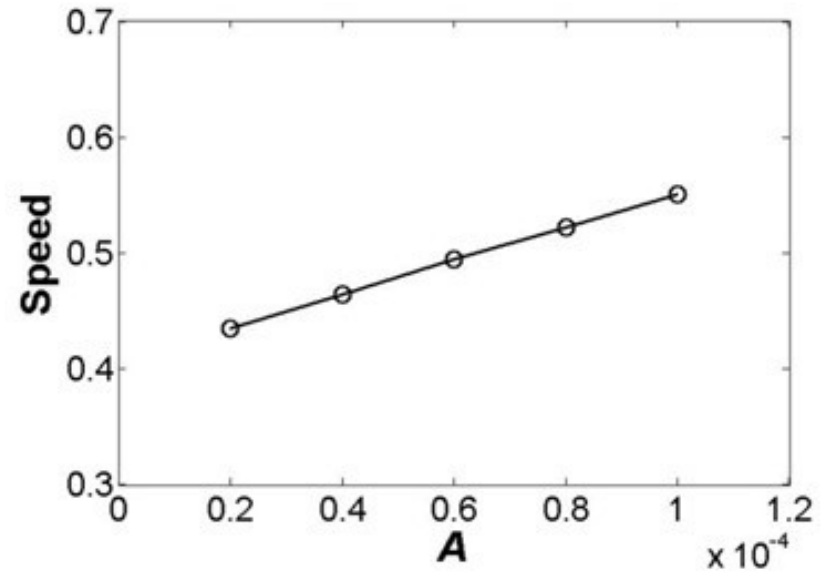

(a)

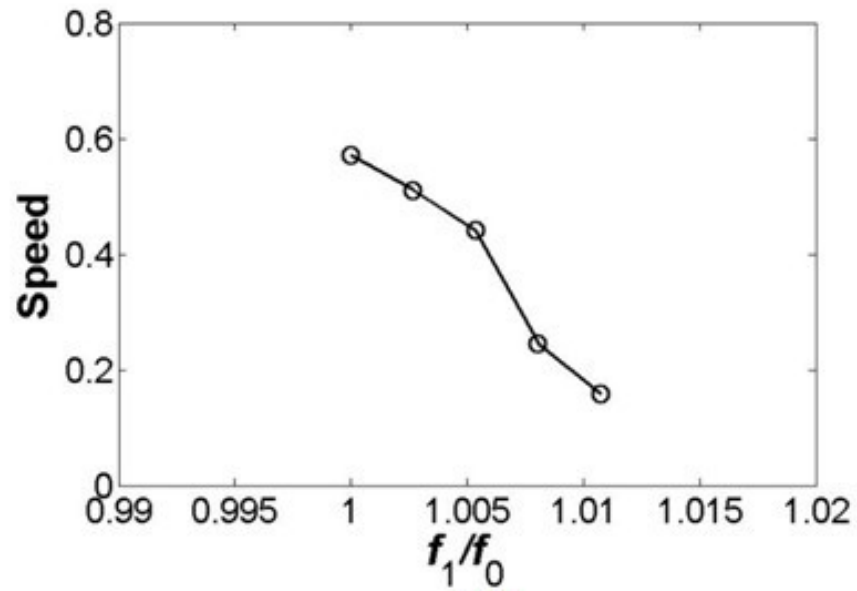

(b)

Figure 4.18. Effect of amplitude and frequency of external forcing on the velocities of phase kinks in the photosensitive Oregonator model. Simulation conditions are the same as Fig. 4.17. In addition, the forcing frequency $f_{1}$ for all simulations in (a) is equal to the natural frequency $f_{0}$ $=0.01662$; the forcing amplitude for all simulations in (b) is 0.0002 . 


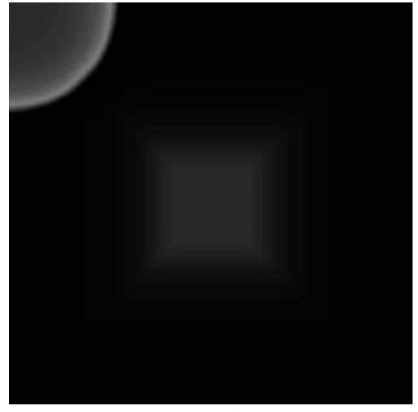

(a)

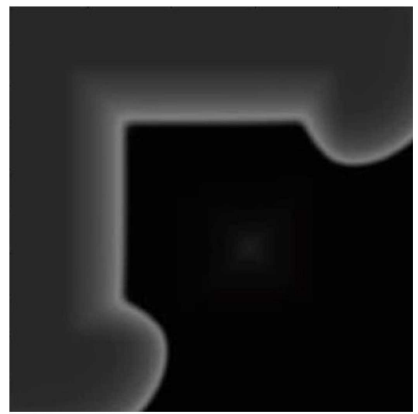

(d)

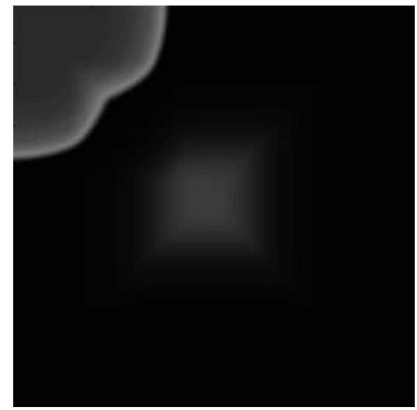

(b)

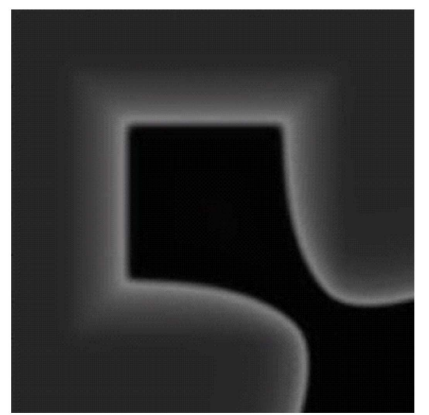

(e)

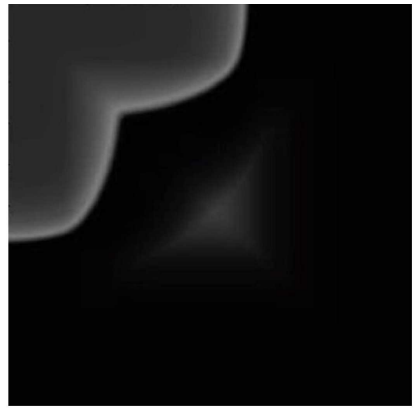

(c)

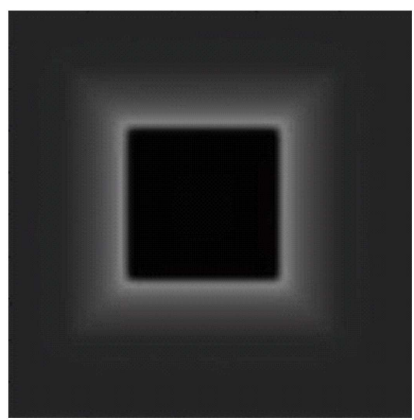

(f)

Figure 4.19. Trap of a phase kink in the photosensitive Oregonator model. Simulation conditions: the numerical size $=1000 \times 1000, d x=0.15, d t=0.004, q=0.002, \epsilon=0.05, f=2.1$, and $D_{u}=$ 1.0. The step number of the simulation $=1 \times 10^{5}$. The background illuminaition $\phi_{0}=0.0005$, and the natural frequency $f_{0}=0.1662$. The pacemaker is created by initially using $\phi=0.00004$ in a specific small region. Outside the trap region, the periodic amplitude $=0.0002$ and the forcing frequency $f_{1}=1.0033 f_{0}=0.1668 \mathrm{~s}^{-1}$; inside the trap region, the periodic amplitude $=0.00008$ and the forcing frequency $f_{1}=f_{0}=0.1662 \mathrm{~s}^{-1}$. 
kink can be observed. Finally, a closed pattern with a constant phase difference is formed.

The setup to trap a phase kink in the photosensitive Oregonator model is the same as in the experiments. A single phase kink generated in the corner will pass through the rectangle trapping region with different forcing. As shown in Fig. 4.19, a closed phase kink pattern forms and it is stationary in time. The simulation result is much better than that seen in the experiments because the medium is completely uniform in the simulations. For example, the frequency of the background oscillation is constant and there are no local heterogeneities, such as dust or gas bubble in the BZ reaction, working as potential pacemakers during the process of forcing. Numerical simulations also show that the frequency of a pacemaker and the size of trap region have an effect on trapping a phase kink. Given the same forcing conditions, the higher frequency of a pacemaker can generate phase kinks propagating with faster speeds, which are difficult to trap.

\subsection{Summary}

A phase kink is the existence of a $\pi$ or $2 \pi$ phase difference in the oscillatory medium. It can be created by coupling two oscillators via a third oscillator. The dynamics of phase kinks has been studied in the photosensitive oscillatory BZ reaction. A wave with a phase difference of $2 \pi$, emitted from a pacemaker, is a phase kink propagating in the oscillatory BZ reaction with 1:1 frequency-locked entrainment.

Both the amplitude and frequency of the external forcing affect the speed of the phase kink in the oscillatory BZ reaction. In the entrainment region, known as Arnold tongue, the effects have been studied using the photosensitive Oregonator model.

In addition, a phenomenon called wave traps has also been observed in both experiments and numerical simulations. In trapping, a region with different forcing conditions is used as the trap. 
When a phase kink propagates through the trap region, the speed changes. Finally, a closed pattern corresponding to a phase difference is formed, indicating that a reversal of the propagation direction of the phase kink has occurred. 


\section{Reference}

[1] J. M. V. Grzybowski, E. E. N. Macau, and T. Yoneyama, "Isochronal synchronization of time delay and delay-coupled chaotic systems," J. Phys. A: Math. Theor. 44, 175103 (2011).

Online Version 68

[2] H. Fujisaka and T. Yamada, "Stability theory of synchronized motion in coupled-oscillator systems," Prog. Theor. Phys. 69, 32-47 (1983).

Online Version 68

[3] L. M. Pecora and T. L. Carroll, "Synchronization in chaotic systems," Phys. Rev. Lett. 64, 821-824 (1990).

Online Version 68

[4] M. G. Rosenblum, A. S. Pikovsky, and J. Kurths, "Phase synchronization of chaotic oscillators," Phys. Rev. Lett. 76, 1804-1807 (1996).

Online Version 68

[5] J. Liu, C. Ye, S. Zhang, and W. Song, "Anti-phase synchronization in coupled map lattices," Phys. Lett. A 274, 27-29 (2000).

Online Version 68

[6] S. Sivaprakasam, I. Pierce, P. Rees, P. S. Spencer, K. A. Shore, and A. Valle, "Inverse synchronization in semiconductor laser diodes," Phys. Rev. A 64, 013805 (2001).

Online Version 68

[7] M. G. Rosenblum, A. S. Pikovsky, and J. Kurths, "From phase to lag synchronization in coupled chaotic oscillators," Phys. Rev. Lett. 78, 4193-4196 (1997).

Online Version 68 
[8] L. Kocarev and U. Parlitz, "Generalized synchronization, predictability, and equivalence of unidirectionally coupled dynamical systems," Phys. Rev. Lett. 76, 1816-1819 (1996). Online Version 68

[9] M. A. Zaks, E.-H. Park, M. G. Rosenblum, and J. Kurths, "Alternating locking ratios in imperfect phase synchronization," Phys. Rev. Lett. 82, 4228-4231 (1999). Online Version 68

[10] A. Prasad, "Universal occurrence of mixed-synchronization in counter-rotating nonlinear coupled oscillators," Chaos, Solitons Fractals 43, 42-46 (2010).

Online Version 68, 73

[11] A. Sharma, M. D. Shrimali, A. Prasad, R. Ramaswamy, and U. Feudel, "Phase-flip transition in relay-coupled nonlinear oscillators," Phys. Rev. E 84, 016226 (2011). Online Version 68

[12] A. S. Mikhailov and K. Showalter, "Control of waves, patterns and turbulence in chemical systems," Phys. Rep. 425, 79-194 (2006).

Online Version 68, 69, 71, 76, 91

[13] B. Marts, E. Meron, A. Hagberg, and A. L. Lin, "Resonant and nonresonant patterns in forced oscillators," Chaos 16, 037113-1-8 (2006).

Online Version 69

[14] D. Battogtokh, A. Preusser, and A. S. Mikhailov, "Controlling turbulence in the complex Ginzburg-Landau equation II. two-dimensional systems," Physica D 106, 327-362 (1997). Online Version 69 
[15] G. Veser, F. Mertens, A. S. Mikhailov, and R. Imbihl, "Global coupling in the presence of defects: Synchronization in an oscillatory surface reaction," Phys. Rev. Lett. 71, 935-938 (1993).

Online Version 72

[16] F. Mertens, R. Imbihl, and A. S. Mikhailov, "Breakdown of global coupling in oscillatory chemical reactions," J. Chem. Phys. 99, 8668-8671 (1993).

Online Version 69

[17] O. Rudzick and A. S. Mikhailov, "Front reversals, wave traps, and twisted spirals in periodically forced oscillatory media," Phys. Rev. Lett. 96, 018302-1-4 (2006).

Online Version 69, 70, 72, 83, 91

[18] P. Coullet and K. Emilsson, "Strong resonances of spatially distributed oscillators: A laboratory to study patterns and defects," Physica D 61, 119-131 (1992).

Online Version 70

[19] P. Coullet and K. Emilsson, "Pattern formation in the strong resonant forcing of spatially distributed oscillators," Physica A 188, 190-200 (1992).

Online Version 70

[20] A. L. Lin, M. Bertram, K. Martinez, H. L. Swinney, A. Ardelea, and G. F. Carey, "Resonant phase patterns in a reaction-diffusion system," Phys. Rev. Lett. 84, 4240-4243 (2000). Online Version 71, 73, 91

[21] B. Marts, A. Hagberg, E. Meron, and A. L. Lin, "Bloch-front turbulence in a periodically forced Belousov-Zhabotinsky reaction," Phys. Rev. Lett. 93, 108305-1-4 (2004).

Online Version 71, 73, 91 
[22] M. Argentina, O. Rudzick, and M. G. Velarde, "On the back-firing instability," Chaos 14, $777-783(2004)$.

Online Version 72, 91

[23] H. Chaté, A. Pikovsky, and O. Rudzick, "Forcing oscillatory media: Phase kinks vs. synchronization," Physica D 131, 17-30 (1999).

Online Version 71

[24] Z. Noszticzius, W. Horsthemke, W. D. McCormick, H. L. Swinney, and W. Y. Tam, "Sustained chemical waves in an annular gel reactor: a chemical pinwheel," Nature 329, 619-620 (1987). Online Version 73

[25] B. Neumann, Z. Nagy-Ungvarai, and S. C. Müller, "Interaction between silica gel matrices and the Belousov-Zhabotinsky reaction," Chem. Phys. Lett. 211, 36-40 (1993). Online Version 73

[26] T. Yamaguchi, L. Kuhnert, Z. Nagy-Ungvarai, S. C. Müller, and B. Hess, "Gel systems for the Belousov-Zhabotinskii reaction," J. Phys. Chem. 95, 5831-5837 (1991). Online Version 73

[27] K.-P. Zeyer and F. W. Schneider, "Periodicity and chaos in chemiluminescence: The ruthenium-catalyzed Belousov-Zhabotinsky reaction," J. Phys. Chem. A 102, 9702-9709 (1998).

Online Version 76

[28] M. K. R. Reddy, Z. Nagy-Ungvarai, and S. C. Müller, "Effect of visible light on wave propagation in the ruthenium-catalyzed Belousov-Zhabotinsky reaction," J. Phys. Chem. 98, 12255- 
12259 (1994).

Online Version 77

[29] Y. Kuramoto, Chemical oscillations, waves, and turbulence (Springer-Verlag, New York, 1984).

Online Version 79

[30] M. Stich and A. S. Mikhailov, "Complex pacemakers and wave sinks in heterogeneous oscillatory chemical systems," Z. Phys. Chem. 216, 521-533 (2002).

Online Version 79

[31] S. Kádár, T. Amemiya, and K. Showalter, "Reaction mechanism for light sensitivity of the $\mathrm{Ru}(\mathrm{bpy})_{3}^{2+}$ catalyzed Belousov-Zhabotinsky reaction," J. Phys. Chem. A 101, 8200-8206 (1997).

Online Version 89 


\section{Chapter 5}

\section{Motion Analysis of Self-Propelled Pt-Silica}

\section{Particles in Hydrogen Peroxide Solutions}

There are numerous realizations of self-propelled particles in biological systems, such as the action polymerizing bacteria Listeria monocytogenes in cells and cell extracts and similarly behaving biomimetic polystyrene particles coated with the bacterial protein ActA [1-3]. Recently, nonbiological micro- to nanoscale particles have been investigated that convert chemical energy into translational motion [4]. These systems provide an opportunity to explore mechanisms of chemomechanical energy transduction and offer a link to self-propelled particles in living systems.

A study of self-propelled, Pt-coated polystyrene particles has been reported by Howse et al.

[5]. This study showed that coating one hemisphere of the particle with Pt is sufficient for selfpropulsion in dilute $\mathrm{H}_{2} \mathrm{O}_{2}$ solutions. The propulsion velocity obtained from the mean-squared displacement of the asymmetrically coated microspheres, also known as "Janus particles", was found to increase with increasing hydrogen peroxide concentration.

In this chapter, we will carry out experiments with silica particles that are asymmetrically coated with Pt and immersed in $\mathrm{H}_{2} \mathrm{O}_{2}$ solutions, similar to the system studied by Howse et al. 
[5]. However, the focus of our study is on the particle orientation with respect to the direction of motion, which we investigate with velocity autocorrelation and propulsion direction analyses.

\subsection{Introduction}

Self-propulsion is an essential feature of living systems [6]. For example, the bacteria, E. coli, obtain propulsion towards or away from environmental stimuli by motion of long flagella and employing a 'run and tumble' strategy. These biological systems have inspired the creation of artificial self-propelled particles. In order to achieve an artificial microscale or nanoscale object that is able to propel itself in a purposeful way, one must consider how to overcome the effects of Brownian motion. Brownian motion refers to the random movement of particles immersed in a fluid (liquid or gas). This random movement was discovered in 1827 by the botanist Robert Brown while studying pollen grains in water using a microscope.

A particularly appealing strategy for propelling small objects is to take advantage of chemical reactions [7]. In early studies, Whitesides and co-workers [8] investigated the propulsion of millimeter-scale objects by the catalytic decomposition of hydrogen peroxide on a platinum surface. More recently, Mallouk and co-workers [4, 9-11] developed and characterized self-propelled asymmetric nanorods, prepared by the electrodeposition of $\mathrm{Au}$ and Pt. When the rods were suspended in dilute solutions of $\mathrm{H}_{2} \mathrm{O}_{2}$, video microscopy revealed that they were propelled generally in the direction of the $\mathrm{Pt}$ end of the rod. This propulsion direction was surprising in view of the study by Whitesides and co-workers [8] in which the motion generally occurred in a direction away from the Pt-coated region, with the propulsion depending on the production of $\mathrm{O}_{2}$ bubbles from the decomposition of $\mathrm{H}_{2} \mathrm{O}_{2}$. Mallouk and co-workers [4, 9-11] proposed that the nanorods, composed of disparate metals in contact with one another, electrochemically generate a localized 
electrophoretic and proton field that induces motion of the rod through the surrounding fluid. Extensive studies by Mallouk, Sen, and co-workers [4, 7, 11-17], Ozin and co-workers [18-20], and Velegol and co-workers $[21,22]$ have identified a number of metallic nanorod systems that convert the fuel molecules hydrogen peroxide and hydrazine into translational and rotational motion. Optical microscopy of Pt-Au nanorods has allowed directionality coefficient analysis, which suggests that directionality is lost at low $(5.0 \%)$ hydrogen peroxide concentrations [8]. The direction of motion relative to the orientation of the particle was not determined in the studies of asymmetrically Pt-coated polystyrene particles by Howse et al. [5].

The dynamical behavior of the self-propelled particles is controlled by the rotational and translational contributions to the motion of the particles. Recent studies of self-propelled particles have focused primarily on particle velocity as a function of the concentration of reactant, usually $\mathrm{H}_{2} \mathrm{O}_{2}$, that fuels the propulsion. Theoretical studies of nanodimers by Ruckner and Kapral [23]

showed that the propulsion can be understood in terms of a concentration gradient established by the catalytic production of product and a difference in the forces of molecular collisions with the catalytic and noncatalytic surfaces. In all of the studies of self-propelled particles, a common feature is a structural asymmetry that gives rise to a gradient of the product species. Howse et al. [5] proposed a mechanism involving diffusiophoresis, in which spatially asymmetric production of product molecules leads to propulsion of the particles [5, 24, 25].

\subsection{Experimental Setup}

Suspensions of half-coated Pt-silica particles in $\mathrm{H}_{2} \mathrm{O}_{2}$ solutions are easily prepared and offer a convenient experimental system for studies of particle self-propulsion. Our study has utilized particles in which effectively two-dimensional motion is exhibited along the surface of a glass slide. 
The $1 \mu \mathrm{m}$ Pt-silica particles in our experiments have a higher density than the aqueous $\mathrm{H}_{2} \mathrm{O}_{2}$ solutions and settle to the bottom of the thin layer of solution.

The particle speeds are likely affected by particle-surface interactions, as the $1 \mu \mathrm{m}$ particles move in the boundary layer at the glass microscope slide surface. However, such interactions do not affect the correlation of particle motion with particle orientation, and the effectively two dimensional motion allows accurate characterization of the particle movement and orientation. In addition, the high extinction coefficient of the thin metal coating together with the illumination focusing by the uncoated silica allows the particle orientation to be continuously monitored during its self-propelled motion. With the location of the particle determined frame to frame, the measurements permit a detailed analysis of the particle orientation and direction of motion. The detailed preparation of the particles and the experimental procedures are described below.

\subsubsection{Preparation of Silica Particles Half-Coated with Platinum}

A dilute aqueous suspension of washed $1 \mu \mathrm{m}$ silica particles (Bangs Laboratories, $0.96 \mu \mathrm{m}$, $15 \%$ coefficient of variation) was deposited on a coverslip $\left(1.8 \times 1.8 \mathrm{~cm}^{2}\right)$ that had been cleaned in Piranha solution (1:3 mixture of $30 \% \mathrm{H}_{2} \mathrm{O}_{2}$ and $18 \mathrm{M} \mathrm{H}_{2} \mathrm{SO}_{4}$ ). After evaporation of the bead solution, a predominantly close-packed, single layer of particles was formed on the substrate. A layer of $\mathrm{Pt} \sim 5 \mathrm{~nm}$ thick was subsequently deposited onto the particles using e-beam evaporation (Temescal BJD-2000) at $\sim 10^{-6}$ Torr, producing particles that are approximately half-coated with platinum metal. The half-coated particles were released from the coverslip by sonication in water. 


\subsubsection{Data Collection and Particle Tracking Analysis}

Small $\sim 2 \mu \mathrm{L}$ aliquots of the suspended particle solution were mixed with hydrogen peroxide solution to form particle suspensions with the desired $\mathrm{H}_{2} \mathrm{O}_{2}$ concentrations. Particle motion was captured using an Olympus IX-81 optical microscope with a $100 \times$ oil objective, which was fitted with a Photronics Cascade 512B camera. In order to maximize frame capture rate, small regions were selected from the field of view that contained one or more particles. The particle motion was then captured at the highest possible frame rate. Images were background corrected and segmented using the software ImageJ. The particle motion was tracked using Video Spot Tracker software (v05.20), which provided the 2D position of the particle and the corresponding frame time.

\subsection{Experimental Results}

We have monitored the autonomous motion of Pt-silica particles in hydrogen peroxide solutions using high-speed, high-resolution imaging (up to $150 \mathrm{fps}$ ) and frame-by-frame particle tracking in order to determine the particle orientation relative to the direction of motion. Image segmentation allows the Pt-silica particle symmetry axis to be identified in each frame. We typically track about 20 particles, with images collected at 45-55 fps. An example of the particle motion analysis is shown in Fig. 5.1A, in which three sequential bright-field optical micrographs of a $1 \mu \mathrm{m}$ Pt-silica particle are superimposed. The particle is shown at time intervals of $\sim 650 \mathrm{~ms}$ in three successive positions during its autonomous motion in a solution of $27.3 \% \mathrm{H}_{2} \mathrm{O}_{2}$. The three images were chosen from a series of images taken at $\sim 55 \mathrm{fps}$, with every 36 th frame selected. The bright region of the particle is the result of a lensing effect of the Kohler illumination of the uncoated half of the silica microbead. The dark region stems from the high extinction coefficient of the Pt-coated half of the 


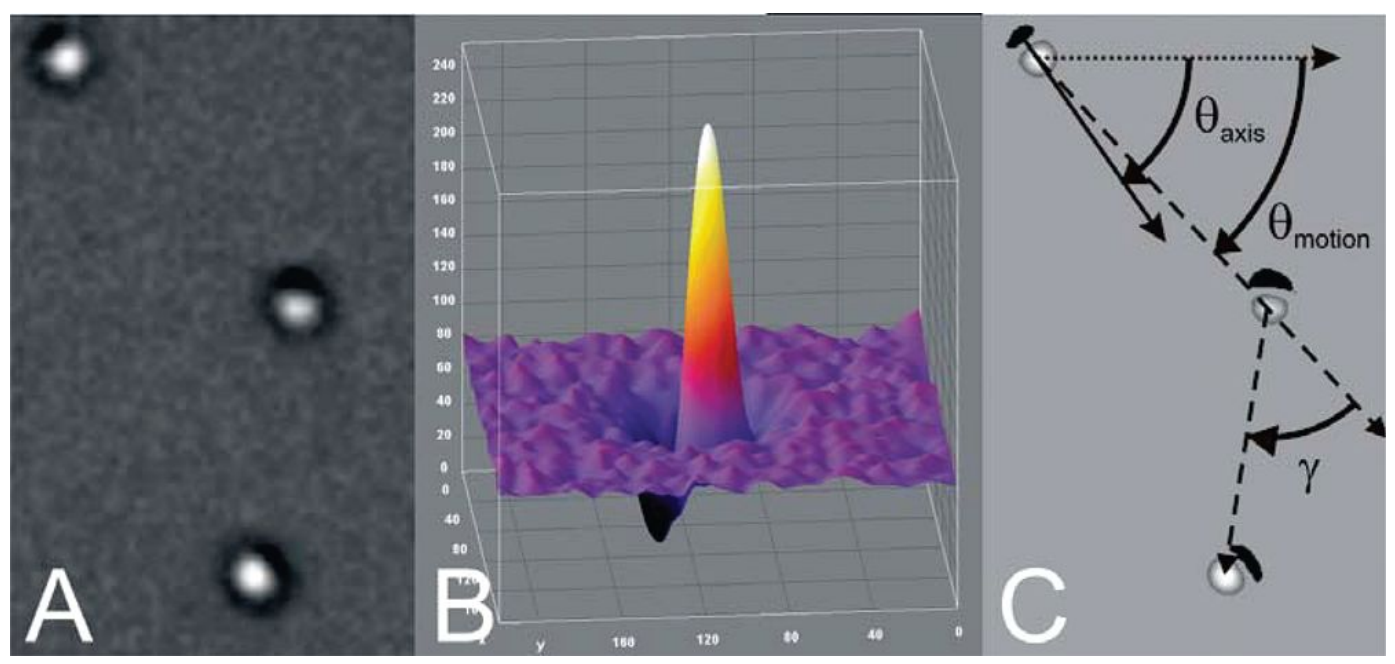

Figure 5.1. (A) Overlay of three sequential optical micrographs of a half-coated Pt-silica particle undergoing autonomous motion. Sequences of micrographs were flat-field corrected to remove background intensities. (B) Surface plot of transmitted light intensity for a single Janus particle. (C) Segmented and background subtracted images of particles from (A) highlighting the silica region (bright) and the Pt-coated region (dark), along with the angles and trajectories that are used in the motion analysis.

particle.

The surface plot in Fig. 5.1B shows the intensity of light transmitted by the particle, with the Pt-coated and silica regions appearing as a valley and a peak, respectively. The orientation of the particle in a particular frame can be determined by considering the extrema of the valley and peak components to be the centers of the Pt-coated and silica regions. Using the background intensity as the reference intensity, each raw image is segmented into two images: one for the Pt-coated region, with intensities less than the reference intensity, and one for the uncoated silica region, with intensities greater than the reference intensity. The two sets of images are then independently tracked to generate an array of $x-y$ location information at subpixel resolutions by kernel matching 


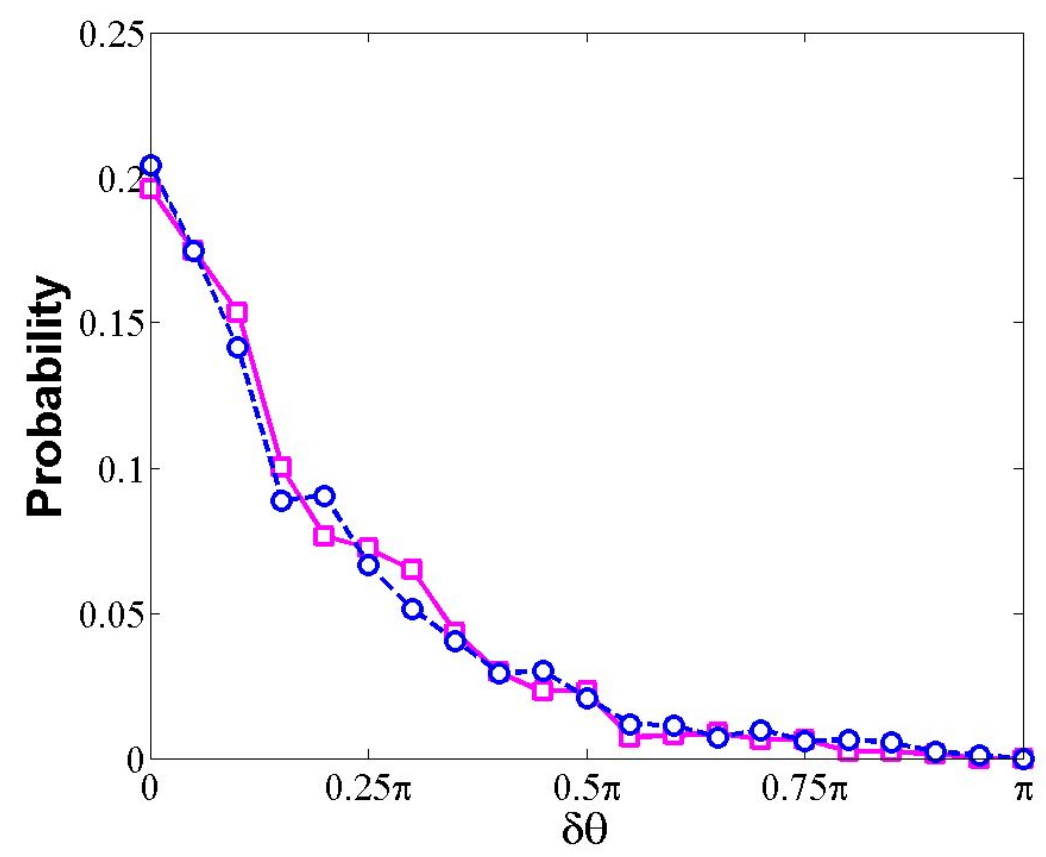

Figure 5.2. Plot of normalized occurrence distribution of $\delta \theta=\left|\theta_{\text {axis }}-\theta_{\text {motion }}\right|$ for half-coated Pt-silica particle motion in $15.0 \%(\square)$ and $27.3 \%(\mathrm{O}) \mathrm{w} / \mathrm{w} \mathrm{H}_{2} \mathrm{O}_{2}$ solutions. Plot constructed from approximately 2000 and 1300 frames of tracked motion, respectively.

with interpolation in ImageJ.

Fig. 5.1C shows segmented images from the raw image data in Fig. 5.1A, with superimposed particle orientation and translation vectors for the motion analysis described below. The combined segmented images clearly show the Pt-coated (black) and the uncoated silica (light gray) regions of the particle. The Pt-coated region of the particle may be smaller due to the vapor deposition procedure; however, the apparent size may also be distorted by the lensing effect of the uncoated silica region. From the $x-y$ position of the uncoated silica region of the particle and the time interval between sequential images, the frame-to-frame trajectory and angle of motion $\theta_{\text {motion }}$ can be determined. In addition, the particle orientation angle $\theta_{\text {axis }}$ is defined by a vector from the intensity minimum corresponding to the Pt-coated side of the particle to the intensity maximum 
corresponding to the uncoated silica side. The trajectory vectors provide the particle velocity, and the directional changes of the particle defined by the angle $\gamma$ can be evaluated from sequential images. These quantities allow an analysis of the particle orientation with respect to its translational motion as a function of time.

A comparison can be made between the particle orientation and the direction of motion from the angle information given by $\theta_{\text {axis }}$ and $\theta_{\text {motion }}$ in Fig. 5.1C. Fig. 5.2 shows a plot of the normalized distribution of the minimum absolute difference between the two angles, $\delta \theta=\left|\theta_{\text {axis }}-\theta_{\text {motion }}\right|$. The value of $\delta \theta$ gives the deviation of the translation direction from the orientation direction of the Pt-silica particle. In the case where the particle travels in a direction exactly in line with its orientation, with the Pt-coated region to the rear, $\delta \theta=0$. The occurrence plot in Fig. 5.2 shows that $\theta_{\text {axis }}$ and $\theta_{\text {motion }}$ coincide to a remarkable degree, consistent with our video observations and the velocity autocorrelation analysis described below. We note that there is little difference in this analysis between the occurrence distribution for particles in $15.0 \%$ and $27.3 \% \mathrm{H}_{2} \mathrm{O}_{2}$ solutions.

The distribution of the directional angle $\gamma$ for uncoated silica particles and Pt-silica particles in water and Pt-silica particles in $\mathrm{H}_{2} \mathrm{O}_{2}$ solutions is shown in Fig. 5.3. Here $\gamma$ is defined as positive for clockwise changes in direction, $\gamma \in(0, \pi]$, and negative for counterclockwise changes in direction, $\gamma \in(-\pi, 0]$, with $\gamma=0$ corresponding to no directional change. The $\gamma$ value can be calculated with three sequential images of a particle according to the law of Cosines. We see that there is virtually no directional preference for silica particles or Pt-silica particles in water (Fig. 5.3a,b), reflecting the random selection of directional changes that accompanies Brownian motion. In contrast, there is a distinct directional preference for half-coated Pt-silica particles in $15.0 \%$ and $27.3 \%$ solutions of $\mathrm{H}_{2} \mathrm{O}_{2}$ (Fig. 5.3c,d), with a narrowing distribution for the solution with the higher concentration. These distributions show that the self-propelled particle travels in the same or nearly the same direction in the subsequent image frame, and the probability of the 

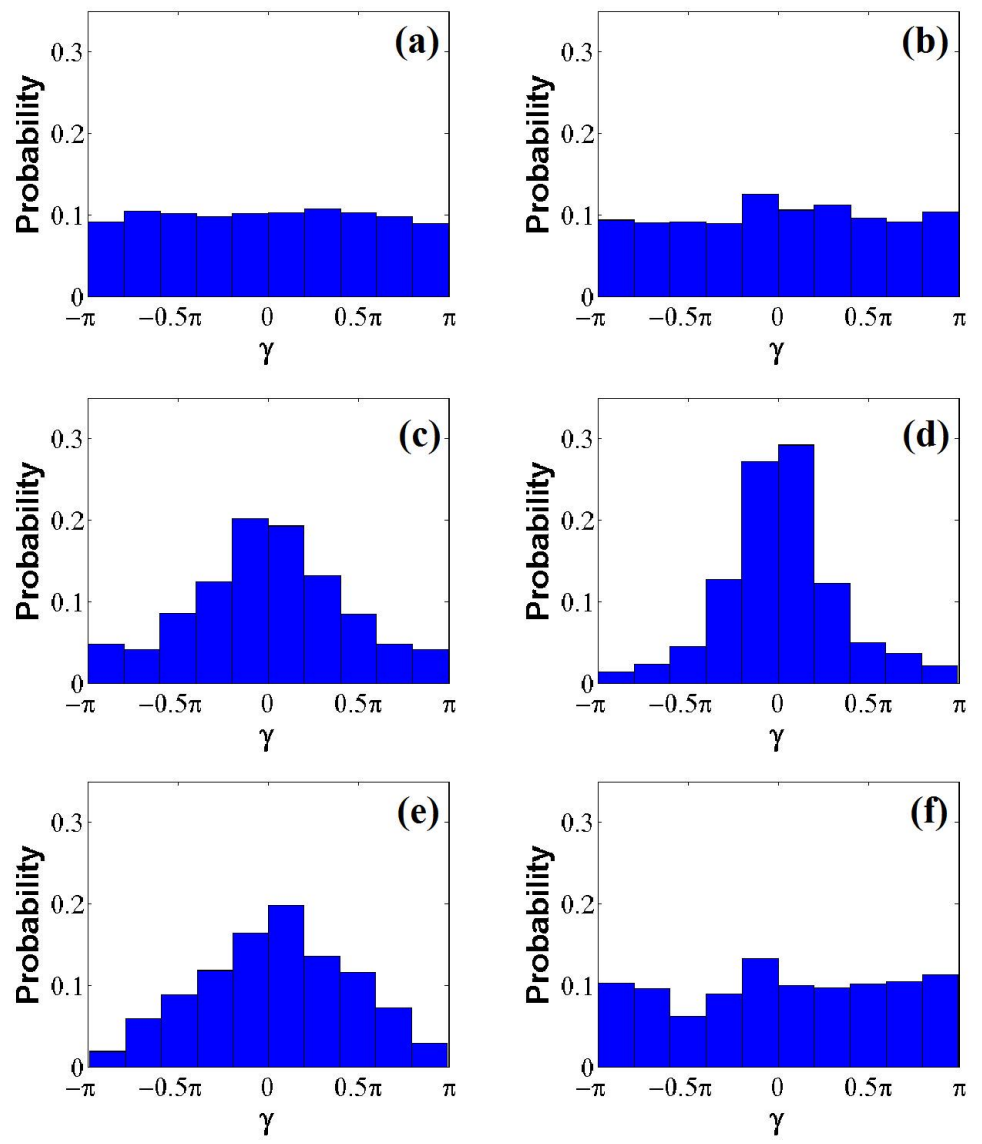

Figure 5.3. Distribution of directional angle $\gamma$ for uncoated silica and half-coated Pt-silica particles in water and $\mathrm{H}_{2} \mathrm{O}_{2}$ solutions. (a) Silica particles in water; (b) half-coated Pt-silica particles in water; (c) half-coated Pt-silica particles in $15.0 \%$ w/w $\mathrm{H}_{2} \mathrm{O}_{2}$ solution; (d-f) half-coated Pt-silica particles in $27.3 \% \mathrm{w} / \mathrm{w} \mathrm{H}_{2} \mathrm{O}_{2}$ solution. For each solution, 3000 frames were analyzed for the particle motion. The observation time interval $\Delta t=0.021$ (a), $0.022(\mathrm{~b}), 0.019$ (c), and $0.018 \mathrm{~s}(\mathrm{~d})$. The effect of observation time interval is shown in (e) with $\Delta t=0.36 \mathrm{~s}$ and (f) with $\Delta t=0.73 \mathrm{~s}$ for half-coated Pt-silica particles in $27.3 \% \mathrm{w} / \mathrm{w} \mathrm{H}_{2} \mathrm{O}_{2}$ solution. 

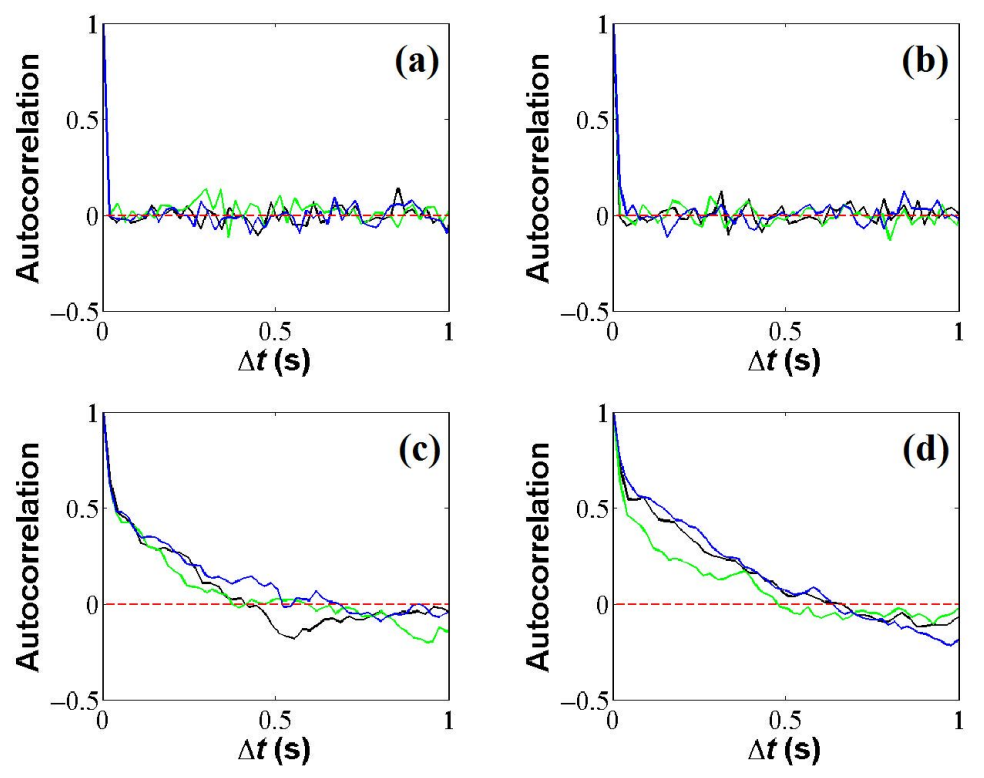

Figure 5.4. Normalized velocity autocorrelation function for uncoated silica and half-coated Ptsilica particles in water and $\mathrm{H}_{2} \mathrm{O}_{2}$ solutions. (a) Silica particles in water; (b) Pt-silica particles in water; (c) Pt-silica particles in $15.0 \% \mathrm{w} / \mathrm{w} \mathrm{H}_{2} \mathrm{O}_{2}$ solution; (d) Pt-silica particles in 27.3\% w/w $\mathrm{H}_{2} \mathrm{O}_{2}$ solution. In each case, the motion of three different particles is analyzed, as shown by the black, blue, and green curves.

directional motion increases with increasing $\mathrm{H}_{2} \mathrm{O}_{2}$ concentration. For Pt-silica particles in 27.3\% solutions of $\mathrm{H}_{2} \mathrm{O}_{2}$, the probability of continuing to travel in nearly the same direction is about 3 times as likely as for these particles in water. The probability of moving at right angles $(\pi / 2$ or $-\pi / 2)$ is about half as likely, and the probability of moving in the opposite direction $(\pi)$ is very unlikely. Figure 5.3d-f shows the distribution of the directional angle $\gamma$ for Pt-silica particles in $27.3 \%$ solutions of $\mathrm{H}_{2} \mathrm{O}_{2}$ with increasing observation time interval $\Delta t$. The distribution of $\gamma$ broadens as $\Delta t$ is increased, indicating that the apparent motion becomes more Brownian-like as the observation time interval becomes comparable to or larger than the rotational diffusion time $\tau_{R}[5]$, as discussed below. 
An analysis of the velocity autocorrelation function (VACF) is consistent with the directional angle distributions. The normalized VACF was calculated by the formula [26]

$$
\chi_{v v}=<v_{p}(0) \cdot v_{p}(t)>=\frac{1}{N_{r}} \sum_{i=0}^{N_{r}} v_{p}(i \Delta t) \cdot v_{p}(i \Delta t+t)
$$

where $v_{p}$ is the particle velocity and $N_{r}$ is the number of realizations.

The normalized VACF for three silica particles and three Pt-silica particles in water are shown in parts a and b of Fig. 5.4, respectively. The velocities of these particles exhibit little if any correlation, with the VACF quickly falling to zero. The VACF for half-coated Pt-silica particles in $15.0 \%$ and $27.3 \%$ solutions of $\mathrm{H}_{2} \mathrm{O}_{2}$ is shown in parts $\mathrm{c}$ and $\mathrm{d}$ of Fig. 5.4, respectively. The analysis is again carried out for three particles in each case. In contrast to the behavior of particles in water, we now see very strong correlations in the particle velocities, with a general trend of the VACF decreasing to zero at $\sim 0.5 \mathrm{~s}$. In both cases, the VACF decreases to slightly negative values after a falloff from large positive values, consistent with our observations of looping behavior at long times described below. In each experiment, a particle was observed for $\sim 5 \mathrm{~s}$, with a camera frame rate of $\sim 50$ fps.

The average speed of the three half-coated Pt-silica particles in water and in $15.0 \%$ and $27.3 \%$ $\mathrm{H}_{2} \mathrm{O}_{2}$ solutions is shown in Fig. 5.5. The speed is determined by the distance traveled between image frames, which, in agreement with the studies of Howse et al. [5], increases with increasing $\mathrm{H}_{2} \mathrm{O}_{2}$ concentration. The average speed of the uncoated silica particles in water is also shown for comparison, which is very close to the speed of the Pt-silica particles in water. Also plotted is the average time at which the VACF falls to zero in each case. These values represent the approximate times when the particle velocities become uncorrelated, with the Pt-silica particles in $\mathrm{H}_{2} \mathrm{O}_{2}$ solutions showing significantly longer correlation times. The corresponding time for the uncoated silica particles in water is also shown, which is essentially the same as the time for the 


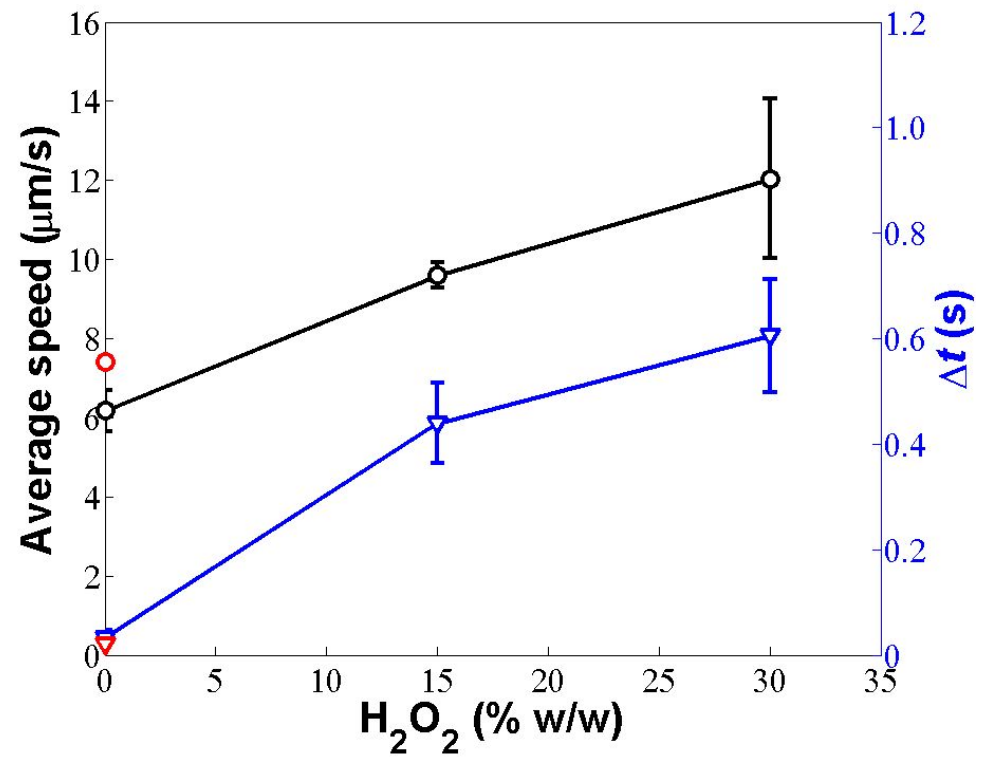

Figure 5.5. Average speed (O) of three half-coated Pt-silica particles analyzed in Fig. 5.4 and the average times in which the velocity autocorrelation function falls to zero $(\nabla)$ in water, $15.0 \%$ $\mathrm{w} / \mathrm{w} \mathrm{H}_{2} \mathrm{O}_{2}$ and $27.3 \% \mathrm{w} / \mathrm{w} \mathrm{H}_{2} \mathrm{O}_{2}$ solutions. The average speed of uncoated silica particles in water $(\mathrm{O}$, red) and the average time that the corresponding autocorrelation function falls to zero $(\nabla$, red). 


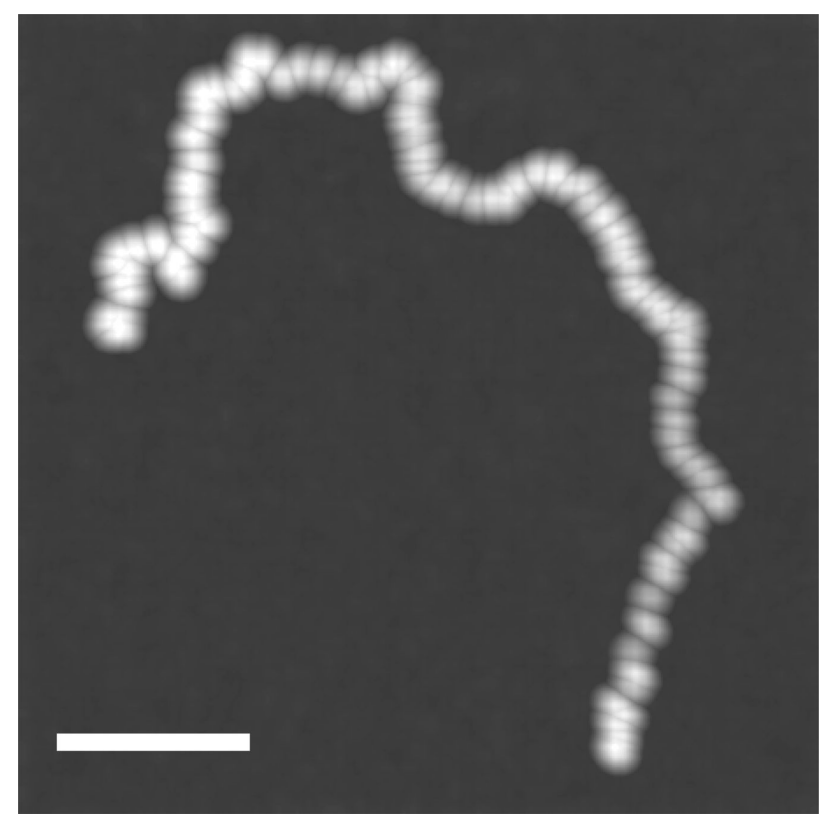

Figure 5.6. Maximum projection of 120 frames of a half-coated Pt-silica particle traveling in a $10.0 \% \mathrm{w} / \mathrm{w} \mathrm{H}_{2} \mathrm{O}_{2}$ solution. The image sequence was background corrected and enlarged before maximum projection. The light gray particle images represent the focal point of the transmitted light through the silica particle, excluding the Pt-coated region and dark edges as seen in Fig. 5.1A, and thus appear slightly smaller than the $1 \mu \mathrm{m}$ nominal bead size. The scale bar is $3 \mu \mathrm{m}$.

Pt-silica particles in water. These correlation times are very close to zero.

The interpretation of the directional angle and velocity autocorrelation analyses described above becomes more intuitive in terms of the high-speed video images. Fig. 5.6 shows an overlay of images of a self-propelled Pt-silica particle in $10.0 \% \mathrm{H}_{2} \mathrm{O}_{2}$ taken at a rate of $\sim 56$ fps. A lower $\mathrm{H}_{2} \mathrm{O}_{2}$ concentration was utilized in this experiment in order to capture Brownian-like motion in one frame area. The composite image shows that the particle typically travels in the same direction in the subsequent frame and continues in the same general direction for several more and sometimes many more frames. Large directional changes occur infrequently but give rise to major changes in the overall particle trajectory. This behavior is in agreement with the directional angle analysis 
shown in Fig. 5.3. We also see that the particle velocity is correlated on short time scales, as shown in the analysis of the VACF in Fig. 5.4; however, on long time scales, the particle turns in wide angles and may travel in the opposite direction. The loss of correlation at $\sim 0.5 \mathrm{~s}$ described in Fig. 5.4 and 5.5 can be seen in the composite image in Fig. 5.6 over sequences of approximately 25-30 frames.

We note that our measurements do not allow us to directly determine the "turning angle" of a particle, i.e., the change in direction that accompanies reorientation of the particle. Our measurements determine the particle position and orientation at each instant a video image is captured, and because directional changes may occur between the moments two successive image frames are captured, the measurements simply correlate the orientation of the particle with the direction of motion. We note below that the observation time interval also affects the value of the translational diffusion coefficient when the time scale of the observation is shorter than the characteristic rotational diffusion time. This is the time for the particle to diffuse $1 \mathrm{rad}$ with the initially observed location as the origin of coordinates.[27].

The directional angle measurements shown in Fig. 5.3 offer insights into the effect of the observation time interval on the apparent motion of the particle. The distributions of the directional angle $\gamma$ for silica and Pt-silica particles in water and Pt-silica particles in $15.0 \%$ and $27.3 \% \mathrm{H}_{2} \mathrm{O}_{2}$ solutions demonstrate that, with an observation time interval of $\Delta t \approx 0.02 \mathrm{~s}$, the self-propelled motion is highly directional compared to Brownian motion. The particles in water display virtually no motion directionality, with an almost uniform distribution of the directional angle $\gamma$ (Fig. 5.3a,b). The motion directionality is random for this purely Brownian behavior. The distribution of the directional angle $\gamma$ is distinctly different for the self-propelled particles in $\mathrm{H}_{2} \mathrm{O}_{2}$ solutions (Fig. 5.3c,d), with the distribution becoming increasingly centered around small values of $\gamma$ with increasing concentration. These distributions for the self-propelled particles indicate that the ro- 
tational diffusion time $\tau_{R}$ is larger than the observation time interval $\Delta t$. The directional angle distributions in Fig. 5.3e,f become more uniform with increasing observation time interval $\Delta t$ for the self-propelle particles. These distributions utilize the same data set for Pt-silica particles in $27.3 \% \mathrm{H}_{2} \mathrm{O}_{2}$ solutions in Fig. 5.3d, with measurements at $\sim 55$ fps. The image sequence was decimate by taking every 20th image for Figure 3e and every 40th image for Figure 3f. We see that the distribution of $\gamma$ becomes less centered and, at the largest $\Delta t$, exhibits characteristics of Brownian motion with a relatively uniform distribution. Hence, with an observation time interval $\Delta t$ that is about 40 times larger than that for observing Brownian motion of Pt-silica particles in water (Fig. 5.3b) or propelled motion in $\mathrm{H}_{2} \mathrm{O}_{2}$ solutions (Fig. 5.3d), the same particles exhibit Brownian-like motion in $\mathrm{H}_{2} \mathrm{O}_{2}$ solutions (Fig. 5.3f).

An alternative approach to the mean-squared displacement for determining the translational diffusion constant $D_{\operatorname{tran}}[5]$ is to examine the step length distribution of a particle in the $x$ and $y$ directions for a given observation time interval $\Delta t[28]$. This approach allows us to take advantage of frame-to-frame measurements of the particle displacement to gain insights into the effect of $\Delta t$ on the apparent motion of the particle. The probability density for a $1 \mathrm{D}$ displacement $\Delta$ is given by

$$
P(\Delta)=A \exp \left(-\Delta^{2} / 2 \sigma^{2}\right)
$$

where $\sigma=\left(2 D_{\operatorname{tran}} \Delta t\right)^{1 / 2}$. The value of $D_{\operatorname{tran}}$ can therefore be determined by fitting Eq. (5.2) to a normal distribution of the step length $\Delta$ for a particular observation time interval $\Delta t$. We demonstrate with this approach that the self-propelled particles exhibit a Gaussian distribution of 1D step length over a wide range of the observation time interval $\Delta t$.

Figure 5.7 shows step length distributions for silica and Pt-silica particles in water and Pt-silica particles in $15.0 \%$ and $27.3 \% \mathrm{H}_{2} \mathrm{O}_{2}$ solutions, with an observation time interval $\Delta t \approx 0.02 \mathrm{~s}$. The 

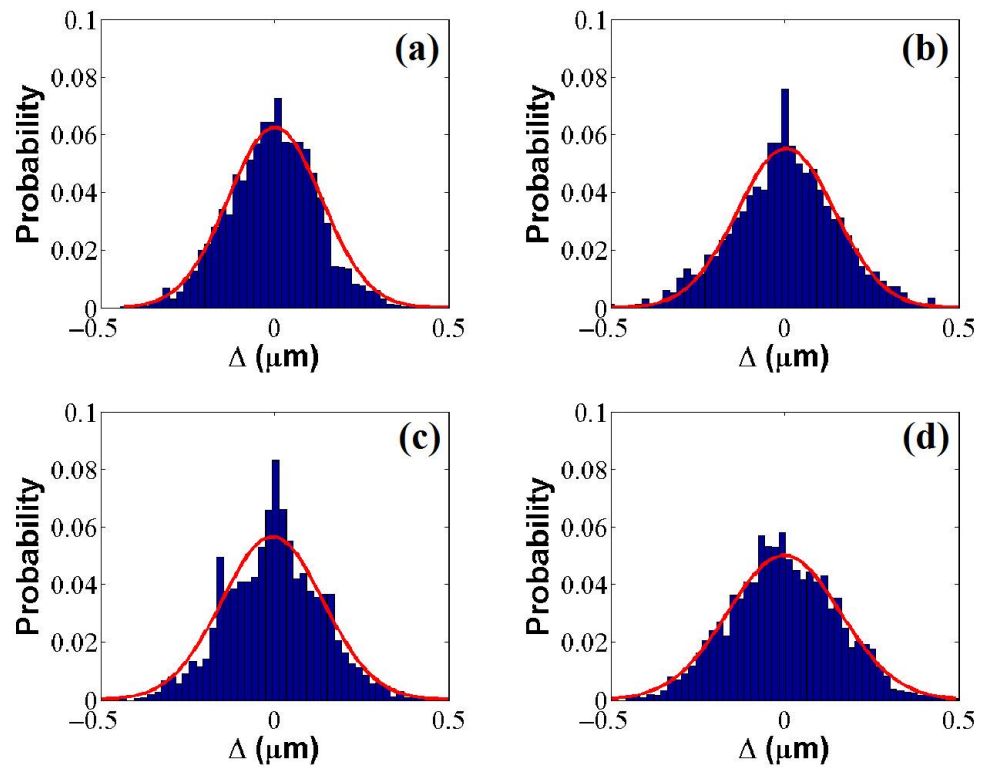

Figure 5.7. Distribution of the step length $\Delta$ for (a) silica and (b) Pt-silica particles in water, and Pt-silica particles in (c) $15.0 \% \mathrm{w} / \mathrm{w}$ and (d) $27.3 \% \mathrm{w} / \mathrm{w} \mathrm{H}_{2} \mathrm{O}_{2}$ solutions. The distributions are plotted for frame-to-frame step lengths in the $\mathrm{x}$ and $\mathrm{y}$ directions and normalized by dividing by the total number of steps (3000). The bar width is $0.02 \mu \mathrm{m}$ in each distribution, and the observation time interval $\Delta t=0.021$ (a), 0.022 (b), 0.018 (c), and $0.019 \mathrm{~s}$ (d). The Gaussian fit is shown by the red line, and the values of $D_{\operatorname{tran}}\left(\mu \mathrm{m}^{2} / \mathrm{s}\right)$ from each fit are (a) $0.42 \pm 0.04$, (b) $0.46 \pm 0.03$, (c) $0.60 \pm 0.06$, and $(\mathrm{d}) 0.66 \pm 0.04$. 

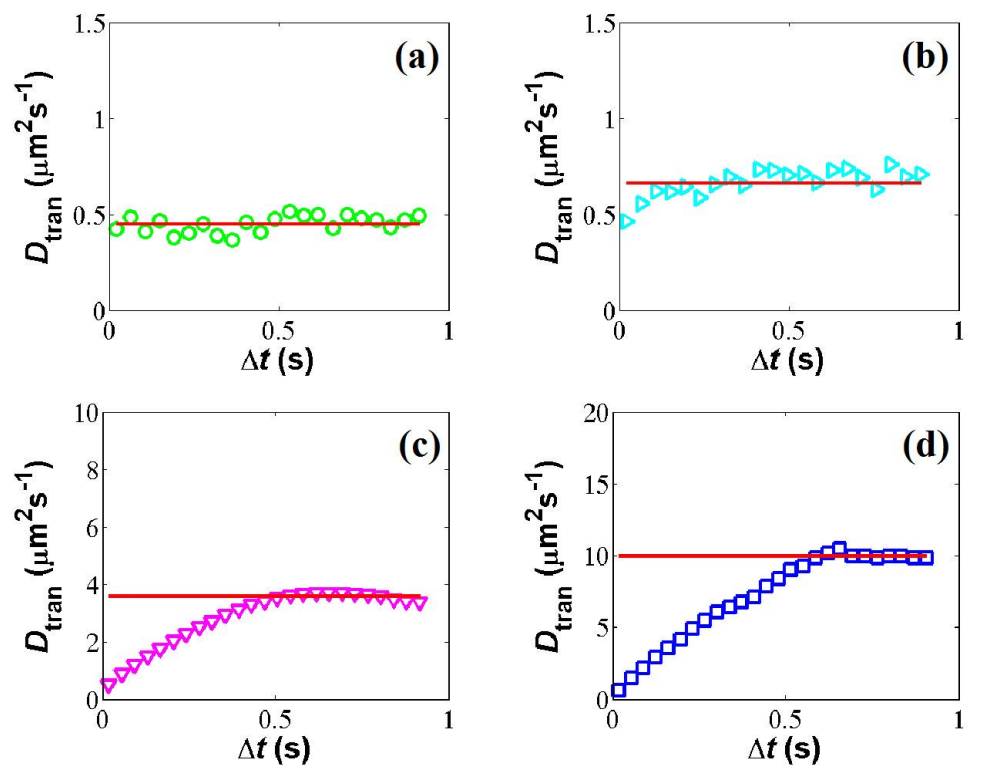

Figure 5.8. Translational diffusion coefficient $D_{\text {tran }}$ as a function of the observation time interval $\Delta t$ for (a) silica and (b) Pt-silica particles in water and Pt-silica particles in (c) $15.0 \% \mathrm{w} / \mathrm{w}$ and (d) $27.3 \% \mathrm{w} / \mathrm{w} \mathrm{H}_{2} \mathrm{O}_{2}$ solutions. The red line in each plot represents the effective translational diffusion constant for Brownian motion. In (c) and (d), an approximate value of $\tau_{R}$ is given by the value of $D_{\text {tran }}$ when it becomes constant, indicating the behavior is Brownian-like with $\Delta t \geq$ $\tau_{R}$. The relative values of $D_{\text {tran }}$ in each plot are given in Table 5.1.

red curves show Gaussian fits for the four cases, with values of $D_{\text {tran }}$ from Fig. 5.7a-d of $0.42 \pm$ $0.04,0.46 \pm 0.03,0.60 \pm 0.06$, and $0.66 \pm 0.04 \mu \mathrm{m}^{2} / \mathrm{s}$, respectively. The dependence of the value of $D_{\text {tran }}$ on the observation time interval $\Delta t$ was investigated by developing new data sets from the data sets for Fig. 5.7 according to $\Delta t_{n}=\Delta t(2 n+1)$ for $n=1,2,3$, etc. The probability density for $1 \mathrm{D}$ displacements $\Delta$ was plotted for each data set corresponding to the incremented observation time interval. The Gaussian fits of these plots, similar to those in Fig. 5.7, provide values of $D_{\text {tran }}$ as a function of $\Delta t$.

The dependence of the translational diffusion constant $D_{\operatorname{tran}}$ on the observation time interval 
Table 5.1. Values of $D_{\text {tran }}, \tau_{R}$, and $V$ from Step Length Probability Distribution

\begin{tabular}{|c|c|c|c|}
\hline & $D_{\text {tran }}\left(\mu m^{2} / s\right)$ & $\tau_{R}(s)$ & $V(\mu m / s)$ \\
\hline $\mathrm{SiO}_{2} /$ water & $0.44 \pm 0.04$ & & \\
$\mathrm{Pt} \mathrm{SiO}_{2} /$ water & $0.68 \pm 0.08$ & & \\
$\mathrm{Pt}-\mathrm{SiO}_{2} / 15.0 \% \mathrm{H}_{2} \mathrm{O}_{2}$ & $3.57 \pm 0.13$ & 0.51 & 4.78 \\
${\mathrm{Pt}-\mathrm{SiO}_{2} / 27.3 \% \mathrm{H}_{2} \mathrm{O}_{2}}$ & $9.97 \pm 0.20$ & 0.62 & 7.73 \\
\hline
\end{tabular}

$\Delta t$ is shown in Fig. 5.8 for silica and Pt-silica particles in water and Pt-silica particles in $15.0 \%$ and $27.3 \% \mathrm{H}_{2} \mathrm{O}_{2}$ solutions. We see in Fig. 5.8a,b that the value of $D_{\text {tran }}$ is independent of $\Delta t$ for the purely Brownian motion of silica and Pt-silica particles in water. The leasts-quares fits of the values, shown by the red line, give similarly small values of $D_{\operatorname{tran}}$ for the particles in water $(0.44$ \pm 0.04 and $0.68 \pm 0.08 \mu \mathrm{m}^{2} / \mathrm{s}$ ). Figure $5.8 \mathrm{c}, \mathrm{d}$ shows that the value of $D_{\text {tran }}$ for Pt-silica particles is dependent on the observation time interval for values of $\Delta t \leq 0.51 \mathrm{~s}$ for $15.0 \% \mathrm{H}_{2} \mathrm{O}_{2}$ solutions and $\Delta t \leq 0.62 \mathrm{~s}$ for $27.3 \% \mathrm{H}_{2} \mathrm{O}_{2}$ solutions. Above these values of $\Delta t$, the value of $D_{\text {tran }}$ becomes independent of the observation time interval, much like the Brownian motion of the particles in water but with significantly larger $D_{\text {tran }}$ values $\left(3.57 \pm 0.13\right.$ and $\left.9.97 \pm 0.20 \mu \mathrm{m}^{2} / \mathrm{s}\right)$.

These data are consistent with the values calculated by the theoretical expressions. The translational diffusion constant for a purely Brownian particle of radius $R$ in water is $D_{\text {tran }}=k_{B} \mathrm{~T} /(6 \pi \eta \mathrm{R})$, where $k_{B} \mathrm{~T}$ is the thermal energy and $\eta$ is the viscosity of water. The particle will also undergo rotational diffusion with a characteristic time scale of $\tau_{R}=\left(8 \pi \eta \mathrm{R}^{3}\right) / k_{B} \mathrm{~T}[5]$. Thus, the theoretical translational diffusion $D_{\text {tran }}$ and rotational diffusion time $\tau_{R}$ of Brownian motion for our silica particles in water are respectively $0.511 \mu \mathrm{m}^{2} / \mathrm{s}$ and $0.601 \mathrm{~s}$, where $R=0.48 \times 10^{-6} \mathrm{~m}, \eta=0.89$ $\mathrm{cP}=0.89 \times 10^{-3} \mathrm{P}, \mathrm{T}=298.0 \mathrm{~K}$, and $k_{B}=1.38 \times 10^{-23} \mathrm{~J} \mathrm{~K}^{-1}$. 
Howse et al. [5] demonstrated that the motion of self-propelled particles can be characterized as directed propulsion for $\Delta t<\tau_{R}$, with an effective diffusion constant $D_{\operatorname{tran}}=D+\frac{1}{4} V^{2} \Delta t$, and Brownian-like for $\Delta t>\tau_{R}$, with $D_{\text {tran }}=D+\frac{1}{4} V^{2} \tau_{R}$, where $V$ is the particle speed arising from the propulsion. A transition in $D_{\text {tran }}$ should therefore be observed as the observation time interval $\Delta t$ is increased from below to above the rotational diffusion time $\tau_{R}$. We see this crossover in behavior in Fig. 5.8c and 5.8d, where the value of $D_{\text {tran }}$ for the motion of Pt-silica particles in $15.0 \%$ and $27.3 \% \mathrm{H}_{2} \mathrm{O}_{2}$ solutions is a linear function of $\Delta t$ for $\Delta t<\tau_{R}$ and is independent of $\Delta t$ for $\Delta t>\tau_{R}$. We can therefore estimate the value of $\tau_{R}$ as the point where $D_{\text {tran }}$ plateaus to a constant value in each case. The value of $\tau_{R}$ increases only slightly, from $\sim 0.51$ to $\sim 0.62 \mathrm{~s}$, with increasing $\mathrm{H}_{2} \mathrm{O}_{2}$ concentration.

The values of $D_{\operatorname{tran}}, \tau_{R}$, and $V$ obtained from the analysis of the $1 \mathrm{D}$ step length distribution in Fig. 5.8 are listed in Table 5.1. The value of $V$ can be calculated from the relation $D_{\text {tran }}$ $=D+\frac{1}{4} V^{2} \tau_{R}$, where $D$ is the diffusion constant for purely Brownian motion of the Pt-silica particles in water. We see that the value of $D_{\text {tran }}$ rises dramatically for the Pt-silica particles in $\mathrm{H}_{2} \mathrm{O}_{2}$ solutions compared to the value of $D_{\text {tran }}$ for the particles in water, and that the value increases with increasing $\mathrm{H}_{2} \mathrm{O}_{2}$ concentration. The value of $V$ also increases with increasing $\mathrm{H}_{2} \mathrm{O}_{2}$ concentration, while the value of $\tau_{R}$ changes only slightly. For comparison, the values of $D_{\operatorname{tran}}, \tau_{R}$, and $V$ obtained from measurements of the mean-squared displacement are listed in Table 5.2.

The analysis based on the step length probability distribution is consistent with the analyses of the VACF and the directional angle distribution. We see in Fig. 5.4 that the velocity correlation falls to zero at times that are roughly the same as the characteristic rotational diffusion time $\tau_{R}$. Hence, at time intervals less than $\tau_{R}$, velocities are correlated by the effects of directed propulsion, and at time intervals greater than $\tau_{R}$, the correlations are lost due to rotational motion. The directional angle distributions in Fig. 5.3c-f also provide compelling evidence for the crossover from 
Table 5.2. Values of $D_{\text {tran }}, \tau_{R}$, and $V$ from Mean-Squared Displacement

\begin{tabular}{|c|c|c|c|}
\hline & $D_{\text {tran }}\left(\mu m^{2} / s\right)$ & $\tau_{R}(s)$ & $V(\mu m / s)$ \\
\hline $\mathrm{SiO}_{2} /$ water & $0.48 \pm 0.01$ & & \\
$\mathrm{Pt}^{-\mathrm{SiO}_{2} / \text { water }}$ & $0.75 \pm 0.03$ & & \\
$\mathrm{Pt}-\mathrm{SiO}_{2} / 15.0 \% \mathrm{H}_{2} \mathrm{O}_{2}$ & $5.48 \pm 0.10$ & 0.53 & 6.14 \\
${\mathrm{Pt}-\mathrm{SiO}_{2} / 27.3 \% \mathrm{H}_{2} \mathrm{O}_{2}}$ & $9.80 \pm 0.32$ & 0.59 & 7.77 \\
\hline
\end{tabular}

directed propulsion to Brownian-like behavior with increasing observation time interval. We see in Fig. 5.3a,b that no directional preference is displayed in the directional angle distributions for silica and Pt-silica particles in water. Even though the observation time interval is smaller than the value of $\tau_{R}$ in this case, we do not expect to see any directional preference in purely Brownian motion, and we see in Fig. 5.8a,b that the value of $D_{\text {tran }}$ is independent of the observation time interval $\Delta t$. For the same observation time interval, however, we see in Fig. 5.3c,d a strong directional preference of the Pt-silica particles in $\mathrm{H}_{2} \mathrm{O}_{2}$ solutions, characteristic of directed propulsion with $\Delta t<\tau_{R}$. As the value of $\Delta t$ is increased in Fig. 5.3e and 5.3f, we find the directional preference is diminished, and for $\Delta t>\tau_{R}$, Brownian-like behavior is exhibited.

\subsection{Summary}

Self-propelled Pt-silica particles in $\mathrm{H}_{2} \mathrm{O}_{2}$ solutions offer a convenient system for the characterization of chemically powered translational motion. In this study, we have found that the observation time scale may correspond to a regime of Brownian-like motion, where the observation occurs on a longer time scale than the rotational diffusion time, or to a regime of directed propulsion, where the observation occurs on a shorter time scale than the rotational diffusion time. This 
crossover in observed behavior with observation time interval allows important parameters to be determined, such as the rotational diffusion time $\tau_{R}$, the effective translational diffusion coefficient $D_{\text {tran }}$, and the velocity derived from the chemical propulsion $\mathrm{V}$.

This system also offers the potential, in future experiments, for further exploration of factors influencing the motion of self-propelled particles, such as imposed temperature, concentration, or viscosity gradients. In addition, the system offers an opportunity to compare the features of nonbiological chemical propulsion to those of self-propelled particles in living systems. 


\section{Reference}

[1] D. Pantaloni, C. L. Clainche, and M.-F. Carlier, "Mechanism of actin-based motility," Science 292, 1502-1506 (2001).

Online Version 104

[2] L. A. Cameron, M. J. Footer, A. van Oudenaarden, and J. A. Theriot, "Motility of acta protein-coated microspheres driven by actin polymerization," Proc. Natl. Acad. Sci. U. S. A. 96, 4908-4913 (1999).

Online Version

[3] A. Bernheim-Groswasser, S. Wiesner, R. M. Golsteyn, M. F. Carlier, and C. Sykes, "The dynamics of actin-based motility depend on surface parameters," Nature 417, 308-311 (2002). Online Version 104

[4] W. F. Paxton, K. C. Kistler, C. C. Olmeda, A. Sen, S. K. St Angelo, Y. Y. Cao, T. E. Mallouk, P. E. Lammert, and V. H. Crespi, "Catalytic nanomotors: Autonomous movement of striped nanorods," J. Am. Chem. Soc. 126, 13424-13431 (2004).

Online Version 104, 105, 106

[5] J. R. Howse, R. A. L. Jones, A. J. Ryan, T. Gough, R. Vafabakhsh, and R. Golestanian, "Self-motile colloidal particles: From directed propulsion to random walk," Phys. Rev. Lett. 99, $048102(2007)$.

Online Version 104, 105, 106, 113, 114, 118, 121, 122

[6] A. Czirok, H. Stanley, and T. Vicsek, "Spontaneously ordered motion of self-propelled particles," J. Phys. A 30A, 1375-1385 (1997). 105 
[7] W. F. Paxton, P. T. Baker, T. R. Kline, Y. Wang, T. E. Mallouk, and A. Sen, "Catalytically induced electrokinetics for motors and micropumps," J. Am. Chem. Soc. 128, 14881-14888 (2006).

Online Version 105, 106

[8] R. F. Ismagilov, A. Schwartz, N. Bowden, and G. M. Whitesides, "Autonomous movement and self-assembly," Angew. Chem. Int. Ed. Engl. 41, 652-654 (2002).

Online Version 105, 106

[9] W. F. Paxton, A. Sen, and T. E. Mallouk, "Motility of catalytic nanoparticles through selfgenerated forces," Chem. Eur. J. 11, 6462-6470 (2005).

Online Version 105

[10] Y. Wang, R. M. Hernandez, D. J. Bartlett, J. J. M. Bingham, T. R. Kline, A. Sen, and T. E. Mallouk, "Bipolar electrochemical mechanism for the propulsion of catalytic nanomotors in hydrogen peroxide solutions," Langmuir 22, 10451-10456 (2006).

Online Version

[11] T. R. Kline, W. F. Paxton, T. E. Mallouk, and A. Sen, "Catalytic nanomotors: Remotecontrolled autonomous movement of striped metallic nanorods," Angew. Chem. Int. Ed. 44, 744-746 (2005).

Online Version 105, 106

[12] J. M. Catchmark, S. Subramanian, and A. Sen, "Directed rotational motion of microscale objects using interfacial tension gradients continually generated via catalytic reactions," Small 1, 202-206 (2005).

Online Version 
[13] P. Dhar, T. M. Fischer, Y. Wang, T. E. Mallouk, W. F. Paxton, and A. Sen, "Autonomously moving nanorods at a viscous interface," Nano Lett. 6, 66-72 (2006).

Online Version

[14] P. Dhar, Y. Cao, T. Kline, P. Pal, C. Swayne, T. M. Fischer, B. Miller, T. E. Mallouk, A. Sen, and T. H. Johansen, "Autonomously moving local nanoprobes in heterogeneous magnetic fields," J. Phys. Chem. C 111, 3607-3613 (2007).

Online Version

[15] W. F. Paxton, S. Sundararajan, T. E. Mallouk, and A. Sen, "Chemical locomotion," Angew. Chem. Int. Ed. 45, 5420-5429 (2006).

Online Version

[16] T. R. Kline, M. Tian, J. Wang, A. Sen, M. W. H. Chan, and T. E. Mallouk, "Template-grown metal nanowires," Inorg. Chem. 45, 7555-7565 (2006).

Online Version

[17] M. E. Ibele, Y. Wang, T. R. Kline, T. E. Mallouk, and A. Sen, "Hydrazine fuels for bimetallic catalytic microfluidic pumping," J. Am. Chem. Soc. 129, 7762-7763 (2007).

Online Version 106

[18] G. A. Ozin, I. Manners, S. Fournier-Bidoz, and A. Arsenault, "Dream nanomachines," Adv. Mater. 17, 3011-3018 (2005).

Online Version 106

[19] S. Fournier-Bidoz, A. C. Arsenault, I. Manners, and G. A. Ozin, "Synthetic self-propelled nanorotors," Chem. Commun. 441-443 (2005).

Online Version 
[20] L. F. Valadares, Y.-G. Tao, N. S. Zacharia, V. Kitaev, F. Galembeck, R. Kapral, and G. A. Ozin, "Catalytic nanomotors: Self-propelled sphere dimers," Small 6, 565-572 (2010). Online Version 106

[21] Y. Hong, N. M. K. Blackman, N. D. Kopp, A. Sen, and D. Velegol, "Chemotaxis of nonbiological colloidal rods," Phys. Rev. Lett. 99, 178103 (2007).

Online Version 106

[22] T. R. Kline, J. Iwata, P. E. Lammert, T. E. Mallouk, A. Sen, and D. Velegol, "Catalytically driven colloidal patterning and transport," J. Phys. Chem. B 110, 24513-24521 (2006). Online Version 106

[23] G. Ruckner and R. Kapral, "Chemically powered nanodimers," Phys. Rev. Lett. 98, 150603 (2007).

Online Version 106

[24] R. Golestanian, T. B. Liverpool, and A. Ajdari, "Propulsion of a molecular machine by asymmetric distribution of reaction products," Phys. Rev. Lett. 94, 220801 (2005). Online Version 106

[25] R. Golestanian, T. B. Liverpool, and A. Ajdari, "Designing phoretic micro- and nanoswimmers," New J. of Phys. 9, 126 (2007).

Online Version 106

[26] V. Rudyak, G. Kharlamov, and A. Belkin, "The velocity autocorrelation function of nanoparticles in a hard-sphere molecular system," Tech. Phys. Lett. 26, 553-556- (2000). Online Version 114 
[27] Y. Han, A. M. Alsayed, M. Nobili, J. Zhang, T. C. Lubensky, and A. G. Yodh, "Brownian motion of an ellipsoid," Science 314, 626-630 (2006).

Online Version 117

[28] P. Nakroshis, M. Amoroso, J. Legere, and C. Smith, "Measuring Boltzmann's constant using video microscopy of Brownian motion," Am. J. Phys. 71, 568-573 (2003).

Online Version 118 